\title{
MASTER
}

\section{Theory of atomic motion in resonant radiation}

\author{
Richard Joseph Cook \\ (Ph.D. Thesis)
}




\title{
Theory of atomic motion in resonant radiation
}

\author{
Richard Joseph Cook \\ (Ph.D. Thesis)
}

Manuscript date: March 1980

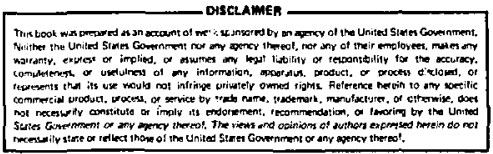

LAWRENCE LIVERMORE LABORATORY University of California $\bullet$ Livermore, California $\bullet 94550$ 
Theory of Atomic Motion in Resonant Radiation

By

RICILARD JOSEPH COOK

B.S. (University of Arizona) 1971

M.S. (Air Force Institute of Technology) 1972

\section{DISSERTATION}

Submitted in partial satisfaction of the requirements for the degree of

DOCTOR OF PHILOSOPHY

in

Engineering-Applied Science

in the

GRADUATE DIVISION

of the

UNIVERSITY OF CALIFORNIA

DAVIS

Approved:

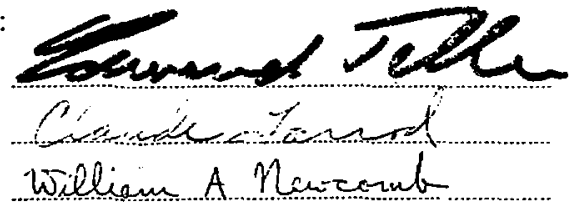

Committae in Charge

Deposited in the University Library 


\section{ABSTRACT}

Atomic motion in resonant and near resonant electromagnetic radiation is investigated theoretically. The exposition begins with a study of atomic motion in a resonant standing light wave, with a view toward isotope separation by selective photodeflection, and proceeds to the investigation of more general problems of atomic motion in resonant radiation. The body of the work consists of six chapters, each of which was prepared as a manuscript for publication in the open literature.

The Schrödinger equation for atomic motion in a resonant standing wave is solved in Chapter 2 in the limit of short atom-field interaction time. It is shown that momentum transfer from the field to the atom in a standing wave proceeds at the rate of induced absorption-emission processes rather than at the spontaneous rate characteristic of momentum transfer in a plane running wave. The resulting rapid deflection process in a standing wave leads to atomic deflections of sufficient magnitude " for isotope separation in a time less than the natural lifetime of the excited atom, and hence circumvents the problem of metastable state trapping encountered in attempts to separate isotopes using a running wave. In Chapter 3 it is shown that a narrow beam of two-level atoms is split by the amplitude gradient of a resonant electronagnetic wave (optical Stern-Gerlach effect), and an experiment is proposed to test this fundamental feature of the resonant interaction. In Chapter 4 it is shown that an exact solution to the Schrödinger equation for atomic motion in a resonant standing wave can be written in terms of Mathieu furctions, 
and that the theory is readily generalized to include the case in which $N$ atomic levels take part in the resonant interaction. A formal analogy between the problem of atomic motion in a standing light wave and diffraction of light by ultrasound is also discussed in this chapter. An alternative approach to the theory of atomic motion in an electromagnetic wave, based on Ehrenfest's theorem and the optical Bloch equations, including effects of spontaneous emission and detuning of the applied field, is developed in Chapter 5. The utility of this theory is illustrated by application to problems of atomic trapping and cooling by the radiation force. The simplicity of calculations in this chapter show that the Ehrenfest-Bloch equations provide a convenient and fruitful framework in which to study such problems. In Chapter 6 the Ehrenfest-Bloch formalism is generalized to take account of laser phase fluctuations and the associated finite linewidth of laser radiation. It is found that fluctuations of the laser radiation alter the predictions of the monochromatic theory oniy when the laser linewidth approaches or exceeds the natural linewidth of the resonant transition, a situation not usually encountered in practice. Finally, in Chapter 7 , the influence on atomic motion of quantum-mechanical fluctuatiuns of the radiation force is investigated. It is shown that fluctuations of the radiation force result from interaction of the fluctuating atomic dipole moment with the applied field as well as from random recoils accompanying spont-neous emission. Atomic motion in the fluctuating radiation force is described by a Fokker-Planck equation, and this equation is used to show that quantum fluctuations place a lower bound on the temperature achievable by radiation cooling, and lead to finite, often short, confinement times for atoms in radiation traps. 
Page

ABSTRACT

TABLE OF CONTENTS

iv

CHAPTER 1. INTRODUCTION AND OVERVIEW

CHAPTER 2. DEFLECTION OF ATOMS BY A RESONANT STANDING ELECTROMAGNETIC WAVE

I. Introduction

II. Theory

III. Numerical Example

CHAPTER 3. THEORY OF ATOMIC MOTION IN A RESONANT ELECTROMAGNET IC WAVE

CHAPTER 4. DIFFRACTION OF ATOMS AND MOLECULES BY A RESONANT STANDING LIGHT HAVE

I. Introduction 40

II. Basic Theory 42

III. Specific Examples $\quad 46$

A. Simple Two-Level Atom 47

B. Two-Level Atom With Degenerate Upper Level 48

C. Truncated Harmonic 0scillator 50

D. Harmonic Oscillator 51

IV. Propagation of Paraxial Atomic Beams 53

V. Diffraction of Atoms by a Simple Standing Wave 55

CHAPTER 5. ATOMIC MOTION IN RESONANT RADIATION: AN APPLICATION OF EHRENFEST'S THEOREM

I. Introduction 64

II. Básic Theory 65

III. Examples $\quad 70$

A. Steady-State Approximation 70

B. Dissipative Force in a Standing Wave

IV. Conclusion 77 
(Table of Contents - Continued)

Page

CHAPTER 6. ATOMIC MOTION IN RESONANT FLUCTUATING

LASER RADIATION

79

I. Introduction 82

II. Field Statistics $\quad 85$

III. Equations of Motion 87

IV. Steady-State Approximation 90

V. Atomic Motion in Broad-Spectrum Radiation 92

CHAPTER 7. QUANTUM-MECHANICAL FLUCTUATIONS OF THE RESONANCE-RADIATION FORCE 97

$\frac{\text { CHAPTER 8. }}{\text { OVERV IEW OF EXPERIMENTS ON ATOMIC MOTION IN }} \quad 109$

CHAPTER 9. CONCLUSION AND RECOMMENDATIONS FOR FUTURE WORK 115

$\begin{array}{ll}\text { ACKNOWLEDGEMENTS } & 118\end{array}$

$\begin{array}{ll}\text { REFERENCES } & 119\end{array}$

$\begin{array}{ll}\text { FIGURES } & 123\end{array}$ 


\section{CHAPTER 1}

\section{INTRODUCTION AND OVERVIEW}

An atom in a resonant or near resonant electromagnetir. wave experiences a force due to momentum transfer from the field. The momentum of one photon is transierred to the atom in the process of absorption, and in the emission process a recoil momentum equal to the negative of the momentum of the emitted photon is imparted to the atom. The momentum transferred to the atom in a single absorption or emission event is quite sma 11 . For example, in the case of sodium, absorption of one photon on the $D_{2} 1$ ine $(\lambda=5890 \AA)$ changes the a tomic momentum by the amount $\Delta P=h / \lambda \approx 10^{-2 ?} \mathrm{~g} \mathrm{~cm} / \mathrm{s}$. If this change of momentum takes place transverse to the direction of propagation of a sodium atomic beam of typical thermal velocity $\left(v \approx 5 \times 10^{4} \mathrm{~cm} / \mathrm{s}\right)$, the beam is deflected through an angle of $50 \mu \mathrm{rad}$ or .003 degrees. Although deflections of this magnitude are observable $e^{1,2}$, one-photon defiections are generally far too small to be of any practical interest. For practical purposes, e.g., isotope separation, angular deflection on the order of a thousand times the above value is required, and consequently repeated absorptionemission processes must be considered.

The simplest photodeflection process capable of proaucing a large deflection is that which occurs in a plane running wave. Absorptionenission processes in a plane wave take place at two distinct rates. Photons are absorbed from and emitted into the plane wave at the stimulated or induced rate $\Omega$, and occasionally photons are spontaneously emitted in random directions at the spontaneous rate $A$ ( $A$ is the Einstein spontaneous emission coefficient). The induced rate is 
determined by the intensity I of the wave and the transition dipole moment $\mu$ of the atom through the re?ation $\Omega=\mu(8 \pi I / c)^{1 / 2} / \hbar$. In a strong field $\Omega$ may exceed $A$ by several orders of magnitude. Now in the induced absorption-emission process the atom gains momentum $\overrightarrow{\hbar k}$ upon absorbing a photon from the wave, but loses an equal momentum due to recoil when the photon is emitted. This occurs in a plane wave because, with only one field mode occupied, induced emission can only add a photon to that mode. It follows that there is no net momentum transfer associated with the induced absorption-emission process in a plane wave, and no average force results from this process. In the process of absorption followed by spontaneous emission, on the other hand, the atom gains monientum $\vec{k} \vec{k}$ upon absorbing a photon, but does not lose this momentum in emission. Photons are spontaneously emitted in random directions, and hence the average recoil momentum delivered to the atom in emission is zero. The atom gains the momentum of one incident photon, on the average, for each spontaneous event. Now the rate of spontaneous events is $A P_{2}$, where $P_{2}$ is the probability that the upper atomic level is occupied (we are assuming that oniy two atomic energy levels take part in the resonant interaction). Tilerefore the rate of momentum transfer to the atom in a plane running wave, i.e., the radiation force is

$$
\overrightarrow{\mathbf{F}}=A P_{2}+\vec{k} \text {. }
$$

This force will be referred to as the spontaneous radiation force. In a strong field the atomic transition is saturated $\left(P_{2}=1 / 2\right)$ and Eq. (1) becomes

$$
\vec{F}=\frac{1}{2} A \pi \vec{k}
$$


If saturating radiation is applied transverse to a sodium atomic beam of velocity $v=5 \times 10^{4} \mathrm{~cm} / \mathrm{s}\left(\lambda=5890 \AA, A=6 \times 10^{7} \mathrm{~s}^{-1}\right)$, the beam experiences a deflection of $1^{\circ}$ in traveling a distance $0.5 \mathrm{~cm}$ in the radiation.

The spontaneous radiation force was first observed by $\mathrm{Frish}^{1}$ in 1933, and was later studied experimentally by Picque and Vialle ${ }^{2}$ using the quasimonochromatic light of a sodium lamp, and by Schieder, Walther and $\ddot{W}$ te $^{3}$ using laser radiation.

Because different isotopes of an element have slightly different transition frequencies (the isotope shift), the spontaneous radiation force can be selectively applied to one isotope in a mixture of isotopes, provided the isotope shift exceeds the linewidth of the transition and the spectral width of the applied field. Therefore it is possiple, in certain favorable cases, to separate isotopes by selectively deflecting the isotope of interest from an atomic beam containing several isotopes. Isotope separation by selective photodeflection using the spontaneous radiation force was first demonstrated by Bernhardt et. al. ${ }^{4}$ for isotopes of barium.

The principle limitation of an isotope separation process based on the spontaneous radiation force is that, for many atoms and molecules, transitions from the upper resonant level to one or more metastable levels terminate the deflection process. Since about one thousand absorption-emission events are required to produce a deflection of sufficient magnitude for practical isotope separation, it is clear that even a small branching ratio from the upper resonant level to a metastable leve1 will remove a large fraction of the atoms from the interaction 
cycle before any significant deflection has taken place. In effect, transitions to a metastable level turn off the radiation force at an early stage of the deflection process, and make this approach to isotope separation impractical in many cases. This problem was encountered by Bernhardt et. $=1.4$ in their work on barium, where it was found that the maximum obtainable momentum transfer on the $5535.7 \AA$ resonance 1 ine was equal to the momentum of 25 photons, i.e., a maximum of 25 absorption emission events were possible before the atom was trapped in the metastable 6s5d ${ }^{1} D_{2}$ state.

The problem of metastable state trapping is associated with the fact that the spontaneous radiation force must act for a time that is long compared to the natural lifetime of the upper resonant level in order to produce a large deflection. If momentum could be transferred to the atom at the induced rate $\Omega$, rather than the spontaneous rate $A$, then in a strong field, where $\Omega$ is several orders of magnitude larger than $A$, it might be possible to produce large deflections in a time that is short compared to the natural lifetime, and thereby avoid the problem of transitions to metastable levels. The work described here began with an attempt to determine under what conditions momentum transfer at the induced rate is possible.

As noted above, there is no net momentum transfer due to induced processes in a plane running wave because the absorbed and emitted photons necessarily have the same momentum. However, if two or more plane running waves propagating in different directions act on the atom simultaneously, as for example in a standing wave (which consists of two counterpropagating running waves), there exists the possibility that a 
photon absorbed from one of the waves (momentum - $\mathrm{kik}_{1}$ ) will be emitted into one of the other waves (momentum $\hbar \vec{k}_{2}$ ) with a resultant transfer of momentum $\Delta \overrightarrow{\vec{P}}=h\left(\vec{k}_{2}-\vec{k}_{1}\right)$ at the induced rate $\Omega$. The average force acting on the atom then depends on the rates of the various induced processes, but in general it is expected that the resulting induced force will be of order of magnitude $F \approx \Omega$ Kk which can be thousands of times larger than the magnitude $\frac{1}{2} A \pi k$ of the spontaneous force.

In a standing wave there are four distinct induced proce,ses as illustrated in Figure 1. The atom can absorb a photon from either of the running waves and emit a photon into the same wave, with no net transfer of momeitum, or the atom can absorb a photon from either wave and emit a photon into the counterpropagating wave, with a transfer of momentum $\Delta P= \pm 2 \pi k$ to the atom. A detailed calculation of atomir motion in a resonant standing wave is required to obcain the resulting distribution of atomic deflections after a large number of induced absorption-emission events have taken place. This calculation is carried out in Chapter 2. It is shown there that, for short interaction time, the probability $P_{n}$ that the atom gains momentum $\Delta P=n \hbar k$ equal to the momentum of $n$ photons is

$$
P_{n}(t)=J_{n}^{2}(\Omega t)
$$

where $J_{n}(x)$ is the Bessel function of order $n, \Omega=\mu(8 \pi I / c)^{1 / 2} /-\hbar$ is the induced rate or Rabi flopping frequency of the two-level atom, and $t$ is the interaction time. The distribution $P_{n}$ is plotted in figure 2(a), 
(b), and (c) for $\Omega t=10,20$, and 30 respectively. $P_{n}$ may also be interpreted as the probability that the atom is deflected through angle $\theta_{n}=n \pi k / P_{z}$, where $P_{z}$ is the initial atomiz momentum. It is seen from Figure 2 that the spread of deflections increases linearly with the interaction time, and the maximum deflection is $\theta_{\max } \approx \Omega t \hbar k / P_{Z}$, indicating that momentum is indeed transferred to the atom at the induced rate $\Omega$. In a typical case it is estimated that deflections of order $\theta_{\max }=3^{\circ}$ can be achieved for an interaction time $t=6 \mathrm{~ns}$. Deflections of this magnitude are sufficient for isotope separation, and the interaction time is less than the natural lifetime of the atomic transition. Therefore this approach to laser isotope separation circumvents the problem of transitions to metastable levels. Such transitions do not have time to occur during the short interaction time of the induced deflection process.

These initial encouraging results lead to a rumber of questions concerning the ultimite efficiency and limitations of the process. The calculation of Chapter 2 is based on an approximation that is valid only in the limit of short interaction time, it considers only the case of exact resonance, it treats the atom as a two-level system, and it ignors spontaneous emission. Therefore some of the questions that remained to be answered were: (1) What is the maximum deflection nbtainable in a resonant standing wave if no constraint is placed on the interaction time?, (2) How is the distribution of atomic deflections a] tered if more than two atomic states take part in the resonant illteraction, e.g., if one or both of the interacting levels is degenerate?, (3) What is the effect on atomic deflections if the frequency of the 
applied field is not exactly equal to the Bohr transition frequency of the atom?, and (4) How does spontaneous emission influence the distribution of atomic deflections when the interaction time is long compared to the natural lifetime of the excited state? A search of the literature revealed that existing theory of atomic motion in resonant radiation was not sufficientiy well developed to answer the above questions. Consequently the scope of the research expanded from the narrow question of the feasibility of isotope separation by photodeflection in a standing wave to more basic questions concerning the motion of atoms and molecules in resonant and near resonant electromagnelic radiation. As significant results were obtained, manuscripts were prepared and submitted for publication in the open literature. The body of this dissertation (Chapters 2 through 7) consists of a collection of these manuscripts. The content of these chapters will now be summarized.

The first manuscript (Chapter 2), entitled "Deflection of Atoms by a Resonant Standing Light Wave," contains the calculation of atomic motion in a standing wave which has already been discussed above. The principle results of this chapter are: (1) that momentum transfer in a standing wave proceeds at the induced rate, and (2) that the resulting rapid deflection process in a strong field overcomes the problem of metastable state trapping.

In the second manuscript (Chapter 3) entitled "Theory of Atomic Motion in a Resonant Electromagnetic Wave," the motion of a two-level atom in an exactly resonant electromagnetic wave of arbitrary amplitude $E(\vec{x})$ is investigated neglecting effects of spontaneous emission. 
A simple calculation shows that the motion of the aton is described by two independent noninterfering wave functions $u_{+}(\vec{x}, t)$ and $u_{-}(\vec{x}, t)$, each of which satisfies a simple tine-dependent Schrödinger equation but with potential energies, $V_{+}(\vec{x})=\frac{1}{2} \mu E(\vec{x})$ and $V_{-}(x)=-\frac{1}{2} \mu E(\vec{x})$, of opposite sign, where $\mu$ is the transition dipole moment of the atom. It follows from this result that a narrow atomic beam is split by the amplitude gradient of the resonant radiation in much the same way as the gradient of a magnetic field splits an atomic beam in the Stern--Gerlach experiment. Finally, an experiment is proposed to test the two-component theory developed in this chapter.

The fourth chapter entitled "Diffraction of Atoms and Molecules by a Resonant Standing Electromagnetic Wave" extends the results of the two preceeding chapters to include the case in which more than two atomic levels strongly interact with the applied field. This work also removes the restriction to short interaction time of chapter 2 by showing that an exact solution of the Schrodinger equation for an atom in a resonant standing wave can be written in terms of Mathieu functions. However, effects of spontaneous emission are still neglected.

When $\mathbf{N}$ atomic levels take part in the resonant interaction, it is found that the motion of the atom is determined by $\mathrm{N}$ independent noninterfering "eigenwaves" $u_{n}(\vec{x}, t), T \leqslant n \leqslant N$, and the nth eigenwave satisfies a time-dependent Schrödinger equation with potential energy $V_{n}(\vec{x})=\frac{1}{2} \mu_{n} E(\vec{x})$, where $\mu_{n}$ is the nth eigenvalue of the matrix of transition moments connecting the $\mathrm{N}$ levels. This again leads to a splitting of an atomic beam by the amplitude gradient of the standing wave, but now the beam splits into $\mathrm{N}$ components. The splitting differs 
from that occurring in the Stern-Gerlach experiment in that the components of the split beam are not necessarily equally spaced, and the probabilities that the atom occupies the various components are not, in general, equal, but rather depend on the structure of the dipole moment matrix.

Since each of the eigenwaves $u_{n}(\vec{x}, t)$ satisfits a Schröinger equation with potential energy $v_{n}(\vec{x})=\frac{1}{2} \mu_{n} E(\vec{x})$, and since the amplitude $E(\vec{x})$ of a plane standing wave is a periodic (sinusoidal) function of position, the deflection of an atom by a resonant standing wave is, in fact, a diffraction process in which the atom is diffracted by the periodic potential it experiences in the standing wave. Chapter 4 shows that diffraction of atoms by a standing light wave is formaliy identical to the problem of diffraction of light by ultrasonic waves in a transparent liquid or solid, and hence many of the results of the theory of diffraction of light by ultrasound are immediately applicable to the problem of diffraction of atoms by a standing light wave.

All of the work described thus far is restricted to the case of exact resonance and ignores spontaneous emission. In practice neither of these approximations is fully justified. The laser frequency is generally not exactly equal to the Bohr transition frequency of the atom, and the approximation of neglecting spontaneous emission is valid only for interaction times that are short compared to the naiviidi lifetime of the excited atom. The latter restriction can be severe at optical frequencies. For example, if the atoms in an atomic beam of typical therma 1 velocity $v=5 \times 10^{4} \mathrm{~cm} / \mathrm{s}$ have a strong optical transition (1ifetime $\tau \sim 10^{-7} \mathrm{sec}$ ), the constraint $t<\tau$ on the interaction time $t$ leads to the constraint $L<v \tau=5 \times 10^{-3} \mathrm{~cm}=50 \mu \mathrm{m}$ on the thickness $\mathrm{L}$ 
of the interaction region. Thus the results of Chapters 2, 3, and 4 can be applied with confidence, at optical frequencies, only when the interaction region is extremely thin. It should be noted that at infrared and microwave frequencies the constraint $t<\tau$ is much less severe, because at these frequencies the natural lifetimes are much longer, but in this case tuning of the radiation to exact resonance becomes more difficult because the natural linewidtis are correspondingly much narrower.

A first step toward a more general theory of atomic motion in electromagnetic radiation, that includes effects of spontaneous emission and detuning of the applied field, is taken in the fifth chapter entitled "Atomic Motion in Resonant Radiation: An Application of Ehrenfest's Theorem." In this work the center-of-mass motion of the atom is calculated on the assumption that the atomic wave packet may be regarded as small compared to the distance over which the applied field changes by a significant amount. It is found that the center-of-mass and internal motions of the atom are coupled. The internal motion is described by a set of optical BToch equations, driven by the electric field at the position of the moving atom, while the radiation force, which drives the center-of-mass motion, depends on the instantaneous value of the Bloch vector. The coupled equations for the internal and translational motions of the atom, which we term the Ehrenfest-Bloch equations, are applied to a number of problems of current experimental interest in this chapter. Specifically, the Ehrenfest-Bloch equations are used to obtain explicit formulas for the radiation force in: 
(1) a plane running wave, (2) a plane standing wave, (3) a combination of running and standing waves, and (4) a coliminated Gaussian beam. Results of these calculations are discussed in connection with recent :1 'sals to trap atoms and molecules by use of the resonant light force $\therefore$ aser radiation. In addition, a formula for the radiation damping force in a weak standing wave, that might be used to cool an atomic vapor to a very low temperature $\left(T \sim 10^{-4} \mathrm{k}\right)$, is derived in this chapter. The simplicity of the calculations presented in this chapter show that an approach based on Ehrenfest's theorem provides a convenient and fruitful framework in which to study such problems.

In Chapter 6 the Ehrenfest-Bloch equations are generalized to take account of phase fluctuations of laser radiation under the sitle "Atomic Motion in Resonant Fluctuating Laser Radiation." All real sources of electromagnetic radiation, including the laser, undergo fluctuations, and hence are not perfectly monochromatic. The finite linewidth of laser radiation is due primarily to phase fluctuations at the source, and the phase fluctuations are well described by the so-called phase-diffusion mode1. The purpose of this chapter is to determine whether or not the finite bandwidth of the laser radiation has any significant influence an atomic motion in laser radiation. The principle result of the chapter is that laser fluctuations alter the predictions of the monochromatic theory only when the laser linewidth approaches or exceeds the natural linewidth of the resonant atomic transition. Since, in practice, the laser linewidth is generally narrower than the natural width of the resonant transition, the effect of laser fluctuations is 
usually quite negligible. It should be noted that the fluctuations considered in this chapter are fluctuations of the classical electromagnetic field, and are entirely different from the quantum-mechanical fluctuations considered in Chapter 7. Quantum fluctuations of the radiation force occur in perfectly monochromatic radiation.

A theory based on Ehrenfest's theorem describes the motior of the centroid of the center-of-mass probability density. It says nothing about the spread of the atomic wave packet about the centroid. Because of this limitation, results of the Ehrenfest-BToch equations can, in some cases, be misleading. For example, the Ehrenfest-Bloch equations predict that the radiation force acting on an atom in a standing wave vanishes when the radiation is tuned to exact resonance, while a more detailed theory (Chapter 3) shows that the atomic trajectory is split by the resonant radiation, in this case. The splitting is symmetric, however, so the centroid of the atomic wave packet is not accelerated. Since the radiation force of Ehrenfest's theorem is defined as the mass of the atom times the acceleration of the centroid, a vanishing radiation force on resonance is certainly consistent with the optical SternGerlach effect, however, this example shcws quite clearly that ïrenfest's theorem provides only limited information about the actual motion of the atom.

A more complete description of atomic motion in resonant radiation, including effects of detuning and spontaneous emission, is developed in the seventh chapter entitled "Quantum-Mechanical Fluctuations of the 
Resonance-Radiation Force." Using the Heisenberg equations for the center-of-mass motion of the atom, the mean radiation force and the quantum-mechanical fluctuations of the radiation force about its mean value are calculated. The mean radiation force causes an average deflection of the atomic trajectory, in agreement with the earlier theory based on Ehrenfest's theorem, while the fluctuating component of the radiation force gives rise to diffusion of atonic momentum. The motion of the atom under the influence of the fluctuating radiation pressure is described by a Fokker-Planck equation in which the mean force and momentum diffusion constant enter as coefficients.

Diffusion of atomic momentum in resonant radiation can be understood from several different points of view. According to one interpretation, diffusion results from repeated splitting of the atomic trajectory as a result of spontaneous emission. If the atom is initially in the ground state, the atomic trajectory is split into two components by the gradient of the applied field amplitude, as described in Chapter 3. Then after each spontaneous emission, which returns the atom to the ground state, each component of the split beam again splits, and repetition of this process leads to diffusion of the atomic momentum. From this point of view, the diffusion is closely related to the optical SternGerlach effect. Alternatively, the diffusion may be regarded as a result of random recoils due to both induced and spontaneous absorptionemission processes. Al though the diffusion has several possible interpretations, the calcuiation of the diffusion coefficient in this chapter does not depend on the interpretation adopted. The diffusion coefficient follows directly from the statistics of the operator force appearing in 
the Heisenberg equations of motion. The principle results of this chapter follow from the fact that the momentum diffusion term in the Fokker-Planck equation tends to increase the kinetic energy of the atom. It is shown that quantum-mechanical fluctuations of the radiation force place a lower bound on the temperature achievable by radiation cooling, and of ten lead to short confinement times for atoms in radiation traps.

Chapter 8 contains a brief overview of some of the more important experiments on atomic motion in resonant radiation, and concluding remarks and recommendations for future work are made in Chapter 9. 
Chapter 2

DEFLECTION OF ATONS BV A RESONANT STANDING ELECTROMAGNETIC WAVE 
Deflection of Atoms by a Resonant Standing Electromagnetic Wave R. J. Cook and A. F. Bernhardt

\author{
University of California \\ Lawrence Livermore Laboratory \\ P. 0. Box 808 , Livermore, California 94550
}

\begin{abstract}
Deflection of an atom due to momentum transfer from a strong resonant standing electromagnetic wave is investigated theoretically in the limit of short atom-field $i$.ueraction time. The transiational and internal motions of the atom are treated quantum-mechanically, while the field is treated classically. It is shown that momentum transfer from a standing wave to an atom proceeds at the induced or Rabi rate, rather than the spontaneous rate characteristic of spontaneous radiation pressure. In a typical case, atomic deflections of order one degree are achieved with $10^{6}$ watt $/ \mathrm{cm}^{2}$ field intensity in a time less than the natural lifetime of the excited atom.
\end{abstract}


I. INTRODUCTION

The use of a resonant electromagnetic wave, or a combination of resonant and static fields, to deflect a beam of neutral atoms has been the subject of renewed interest sirice the advent of high-power tunable lasers. A potential application of laser deflection is to problems of laser isotope separation.

Severa 7 methods of photodeflection have been proposed ${ }^{5-9}$, some of which have been denonstrated experimenta11y $1-4,10,7 ?$. Fiost of these methods require an interaction time that is long compared to the natural lifotime of the excited atoms. This makes the practical application of these methods impossible in many cases because an atom excited by the resonant radiation makes transitions to metastable states which are not affected by the applied field ${ }^{11}$. Such transitions remove atoms from the interaction cycle, and littie or no deflection is produced. The purpose of this paper is to show that in a strong resonant standing wave, significant atomic deflections can occur in a time less than the spontaneous lifetime of the excited atom, and hence, the problem of transitions to metastable levels is circumvented by the speed of the process.

When an atomic beam is irradiated by a strong resonant electromagnetic wave, absorption-emission processes proceed at two distinct rates. Photons are absorbed from and emitted into the applied field at the induced rate $\Omega$, and occasionally photons are spontaneously emitted, in random directions, at the spontaneous rate $A$. Deflection or scattering 
of the atomic beam results when momentum is transfered from the field to the atoms, and the rate of momentum transfer depends on the nature of the applied field.

If the applied field consists of a single plane wave, momentum is transfered to the atoms at the spontaneous rate $A$. This momentum transfer, i.e. radiation pressure, proceeds at the spontaneous rate because absorption followed by induced emission into the same field mode involves no net transfer of momentum, while absorption followed by spontaneous emission transfers an average of one quantum of mementum for each spontaneous event (isotropic spontaneous emission dees not carry away the momentum acquired by the atom through absorption).

If the applied field is composed of two or more plane waves, an atom can absorb a photon from one of the plane waves and induced emission can cause that photon to be emitted into a different plane wave, with a resultant transfer of momentum at the induced rate $\Omega$. Since the induced rate may exceed the spontaneous rate by many orders of magnitude in a strong applied field, it is expected that deflection processes operating at the induced rate will be more efficient and more rapid than processes that operate at the spontaneous rate. In the following we shaii show that momentum transfer in a standing wave proceeds at the induced rate, and that it is this feature of the interaction that gives rise to the rapid deflection mentioned above.

In the model adopted here, the internal motion of the atom is treated as a two-level system. The center-of-mass motion of the atom is treated quantum-mechanically, and the resonant standing wave is treated as a classically prescribed electric field. Analytic solutions of the 
Schrödinger equation are obtained, in the rotating wave approximation, on the assumption that the Doppler width associated with initial beam spread and subsequent atomic deflection is small compared to the frequency width associated with the finite time during which the atom interacts with the resonant radiation.

In the following section, the theory of deflection of an atom by a resonant standing wave is developed and ti.e 6 : itct of finite divergence in a beam of atoms is briefly discussed. In section III the limit of validity of our assumption concerning Doppler effect is examined, and a numerical example is given to illustrate the magnitude of deflections obtainable.

\section{THEORY}

The Hamiltonian for an atom in a classically prescribed electromagnetic field, in the dipole approximation, takes the familiar form

$$
H=P^{2} / 2 M+H_{0}+\vec{\mu} \cdot \vec{E}(\vec{R}, t)
$$

where $P^{2} / 2 M$ is the kinetic energy associated with the center-of-mass momentum $\vec{P}, H_{0}$ is the Hamiltonian for the internal motion of the unperturbed atom, $\vec{\mu}$ is the dipole moment operator, and $\vec{E}(\vec{R}, t)$ is the electric field evaluated at the cante of-mass position $\vec{R}$.

We shall calculate the motion of an atom which starts out moving in the positive $z$ direction, enters a region of resonant radiation at $z=0$, and exits the interaction region at $z=L$. The electric field in the 
interaction region is taken to be a standing wave of the form

$$
\vec{E}(x, t)=2(8 \pi I / c)^{1 / 2} \hat{\varepsilon} \cos k x \cos \omega t
$$

which is equivalent to two plane waves, each of intensity I, counterpropagating along the $x$ axis. The polarization vector $\hat{\varepsilon}$ is a unit vector transverse to the $x$ direction.

For an electric field of this form, only the $x$ coordinate of the center of mass appears in the Hamiltonian. It follows that motion of the atom in the $y$ and $z$ directions is unaffected by the field, and only motion in the $x$ direction is of interest. Elimination of the inessential degrees of freedom yields the Hamiltonian

$$
H=P_{x}{ }^{2} / 2 M+H_{0}-\vec{H} \cdot \vec{E}(x, t)
$$

for atomic motion in the $x$ direction. As the atom moves along the $z$ axis, the interaction term in Equation (3) is switched on as the atom enters the interaction region and is switched off as it leaves this region. Upon exiting the interaction region, the atom has a certain probability density $W(p)$ for momentum $p$ in the $x$ direction. This momentum density determines the probability density for displacement $x$ as $z+\infty$, $P(x)=\left(p_{z} / z\right) W\left(p_{z} x / z\right)$. If the deflections are small $(x / z \ll 1, \theta \approx x / z)$, the probability density for deflection $\theta$ is

$$
P_{\theta}(\theta)=p_{z} W\left(p_{z} \theta\right)
$$

where $p_{z}$ is the $z$ component of atomic momentum. 
To obtain the transverse momentum density $W(p)$, we solve the Schrödinger equation in the momentum representation. We start by writing down the general equations of motion, and then simplify these by using the two-level-atom and rotating-wave approximations. The unperturbed Hamiltonian, $H^{-}=P_{X}{ }^{2} / 2 M+H_{0}$, has eigenvectors $|n, p\rangle=|n>| p>$ and eigenvalues $\varepsilon_{n}(p)=p^{2} / 2 M+E_{n}$, where $\mid n>$ and $E_{n}$ are the eigenvectors and eigenvalues of $H_{0}$, and $|p\rangle$ is the eigenvector of $P_{X}$ with eigenvalue $p$. An arbitrary state vector is expanded as

$$
|\psi\rangle=\sum_{n} \int d p \phi_{n}(p)|n, p\rangle
$$

where $\phi_{n}(p)$ is the amplitude for momentum $p$ and internal energy $E_{n}$. Upon substituting this expansion into the Schrödinger equation, $i \hbar \partial|\psi>/ \partial t=H| \psi>$, and using the orthoriormality of the basis states $|n, p\rangle$, we obtain the equations of motion

$$
i \hbar \frac{\partial \omega_{n}(p)}{\partial t}=\sum_{m} \int d p^{-}\left\langle n, p|H| m, p^{\wedge}\right\rangle \phi_{m}\left(p^{\wedge}\right\rangle .
$$

Evaluation of the matrix elenents $\left\langle n, p|H| m, p^{-}\right\rangle$, using Equations $(2\rangle$ and (3), is straight forward. The explicit equations of motion are

$$
\begin{aligned}
& i \hbar \frac{\partial \phi_{n}(p)}{\partial t}=\varepsilon_{n}(p)_{n}(p) \\
& -\cos \cot \sum_{m} g_{n m}\left[\phi_{m}(p-\hbar k)+\phi_{m}(p+\hbar k)\right]
\end{aligned}
$$

where $g_{n m}=(8 \pi I / C)^{1 / 2}\langle n|\vec{H} \cdot \hat{\varepsilon}| m\rangle$. Equation (7) shows that a change of the excitation state of the atom is accompanied by a transfer of momentum $\pm \hbar k$. Note that $g_{n n}=0$ if the atomic levels are nondegenerate. The transformation 


$$
\phi_{n}(p)=C_{n}(p) \exp \left\{-i \varepsilon_{n}(p) t / \hbar\right\}
$$

with $E_{n}(p)=p^{2} / 2 M+E_{n}$, puts Equation (7) in the form

$$
\begin{aligned}
& i \hbar \frac{\partial C_{n}(p)}{\partial t}=-\cos \omega t \sum_{m} g_{n m} \\
& x\left[C_{m}(p-\hbar k) \exp \left\{-i\left[\omega_{m n}+\delta-\frac{\omega p}{M c}\right] t\right\}\right. \\
& \left.+C_{m}(p+\hbar k) \exp \left\{-i\left[\omega_{m n}+\delta+\frac{\omega p}{M c}\right] t\right\}\right]
\end{aligned}
$$

where $\omega_{n m}=\left(E_{n}-E_{m}\right) / \hbar, \delta=\hbar \omega^{2} / 2 M c^{2}$, and the amplitudes $C_{n}(p)$ are now slowly varying functions of time (interaction picture).

If an atom, initially at rest, absorbs a photon of energy $\hbar_{\omega}$ and momentum $\hbar_{\omega} / c$, the internal energy of the atom increases by the amount $\hbar_{\omega_{m n}}$, its kinetic energy increases by the amount $\left(\hbar_{\omega} / c\right)^{2} / 2 M=\hbar_{\delta}$, and conservation of energy, $\hbar_{\omega}=\hbar_{\omega_{m n}}+\hbar \delta$, shows that the resonant frequency of the transition is $\omega=\omega_{m n}+\delta$. Thus $\delta$ is a frequency shift associated with recoil of the atom. The quantities $\pm \omega p / M c$ are Doppler shifts due to motion of the atom in the $x$ direction. If the interaction time is sufficiently short, then both recoil and Doppler shifts can be neglected. The condition that $\omega \mathrm{pt} / \mathrm{Mc} \ll 1$ has the simple physical meaning that the frequency width associated with the finite transit time of the atom across the field (transit time broadening) is large compared to the accumulated Doppler shift. We make this assumption of short interaction time in the following analysis, and discard exponential factors of the from $\exp \{-i(\delta \pm \omega \mathrm{p} / \mathrm{Mc}) t\}$ in Eq. (9).

We shall consider the case where the applied field is resonant with only a single atom transition $\left(\omega=\omega_{m n}, m=+, n=-\right)$. Then if we neglect all but the two ampiitudes $C_{ \pm}(p)$ involved in the transition (two-level atom approximation) $^{12}$, expand $\cos t$ in exponentials and 
keep only terms on the right in Equation (9) that vary slowly with time (rotating wave approximation) $^{12}$, Equations (9) reduce to

$$
\begin{aligned}
& \dot{i}_{+}(p)=(i \Omega / 2)\left[c_{-}(p-\hbar k)+c_{-}(p+\hbar k)\right] \\
& \dot{c}_{-}(p)=(i \Omega / 2)\left[c_{+}(p-\hbar k)+c_{+}(p+\hbar k)\right]
\end{aligned}
$$

where $\Omega=\left(8 \pi I / c h^{2}\right)^{1 / 2}\langle-|\vec{\mu} \cdot \hat{\varepsilon}|+\rangle$. The phases of $\left.l \pm\right\rangle$ are chosen so that $\Omega$ is real.

Equations (10) may be solved exactly. The transformation

$$
\begin{aligned}
& D_{+}(p)=\left[C_{+}(p)+C_{-}(p)\right] / 2^{1 / 2} \\
& D_{-}(p)=\left[C_{+}(p)-C_{-}(p)\right] / 2^{1 / 2}
\end{aligned}
$$

decouples Equations (10) as

$$
\begin{aligned}
& \dot{D}_{+}(p)=(i \Omega / 2)\left[D_{+}(p-\hbar k)+D_{+}(p+\hbar k)\right] \\
& \dot{D}_{-}(p)=-(i \Omega / 2)\left[D_{-}(p-\hbar k)+D_{-}(p+\hbar k)\right] .
\end{aligned}
$$

Upon substituting the trial solution

$$
D_{ \pm}(p)=\exp \{i s p \pm i a t\}
$$

into Equations (12), we obtain the dispersion relation

$$
\alpha(s)=\Omega \cos (\hbar k s)
$$

for waves in momentum space. Physically acceptable solutions are obtained for all real values of $s$. The general solution of Equations (12) is a superposition of waves 


$$
D_{ \pm}(p, t)=\frac{1}{\sqrt{2 \pi}} \int_{-\infty}^{\infty} a_{ \pm}(s) \exp \{i s p \pm i \alpha(s) t\} d s .
$$

At $t=0$, Equation (15) reduces to a Fourier transform reTation between $a_{ \pm}(s)$ and $D_{ \pm}^{\circ}(p)=D_{ \pm}(p, 0)$. When the inverse of this transform, namely

$$
a_{ \pm}(s)=\frac{1}{\sqrt{2 \pi}} \int_{-\infty}^{\infty} D_{ \pm}^{o}(p) \exp \{-i s p\} d p,
$$

is substituted into Equation (15), we obtain

$$
D_{ \pm}(p, t)=\int_{-\infty}^{\infty} G_{ \pm}(p-p ; t) D_{ \pm}^{o}\left(p^{-}\right) d p ;
$$

where

$$
G_{ \pm}(p, t)=\frac{1}{2 \pi} \int_{-\infty}^{\infty} \exp \left\{ \pm i_{\alpha}(s) t+j p s\right\} d s .
$$

With the help of the dispersion relation, Equation (14), and the identity

$$
e^{ \pm i z \cos \theta}=\sum_{n=-\infty}^{\infty}( \pm i)^{n} J_{n}(z) e^{\mp i n \theta},
$$

the propagators $G_{ \pm}(p, t)$ are readily evaluated as series of Bessel functions

$$
G_{ \pm}(p, t)=\sum_{n=-\infty}^{\infty}( \pm i)^{n} J_{n}(\Omega t) s(p-n \hbar k)
$$

and the general solution, Equation (17), becomes

$$
D_{ \pm}(p, t)=\sum_{n=-\infty}^{\infty}( \pm i)^{n} J_{n}(\Omega t) D_{ \pm}^{o}(p-n \hbar k) .
$$

Consider the case where the atom is in the lower state and has momentum $p=0$ at $t=0$. In this case, $c_{+}^{0}(p)=0, c_{-}^{0}(p)=[\delta(p)]^{1 / 2}$, and from Equations $(17), D_{ \pm}^{0}=[\delta(p) / 2]^{1 / 2}$ (the square root of the $\delta-$ function is used here so that the probability that the atom is in the ground state, namely $P_{-}^{\circ}=\int\left|C_{-}^{\circ}(P)\right|^{2} d P$, is properly nomalized to unity). 
It follows from Equations (11) and (20) that the momentum probability density, $w(p)=\left|C_{+}\right|^{2}+\left|C_{-}\right|^{2}=\left|D_{+}\right|^{2}+\left|D_{-}\right|^{2}$, has the form

$$
W(p, t)=\sum_{n=-\infty}^{\infty} J_{n}^{2}(\Omega t) \delta(p-n \hbar k) .
$$

Equation (21) states that the probability $P_{n}(t)$ that the atom has acquired momentum $n \hbar k(n=0, \pm 1, \pm 2, \cdots)$ is

$$
P_{n}(t)=J_{n}^{2}(\Omega t)
$$

In cases of practical interest, $\Omega t$ is a large number. For $|n|<\Omega t$, the probability $j_{n}^{2}(\Omega t)$ is not a monotonic function of $n$, but tends to increase with $|n|$ and has maxima near $|n|=\Omega t$. For $|n|>\Omega t, J_{n}^{2}(\Omega t)$ decreases rapidiy to zero as $|\mathrm{n}|$ increases. In view of Equation (4), the maximum deflection is $\theta_{\max } \approx \hbar k \Omega t / p_{z}$. This result shows quite clearly that momentum is transfered to the atom at the induced rate $\Omega$.

The mean magnitude of momentum transfered to the atom

$$
\langle|p|\rangle=\sum_{n=-\infty}^{\infty} \hbar k|n| J_{n}^{2}(\Omega t)
$$

can be expressed in closed form ${ }^{73}$

$$
\begin{aligned}
\langle|p|\rangle & =\hbar k(\Omega t)^{2}\left[J_{0}^{2}(\Omega t)+J_{1}^{2}(\Omega t)\right] \\
& -\hbar k \Omega t J_{0}(\Omega t) J_{1}(\Omega t)
\end{aligned}
$$

and approaches the value $\langle|p|\rangle=2 \hbar \mathrm{k} \Omega \mathrm{t} / \pi \approx 0.64 \hbar \mathrm{k} \Omega \mathrm{t}$ as $\Omega \mathrm{t} \rightarrow \infty$. The RMS momentum, at any time $t$, is given by the simple formula $\left[\left\langle p^{2}\right\rangle\right]^{1 / 2}=$ $\hbar_{k \Omega t} / \sqrt{2} \approx 0.71 \hbar k \Omega t .{ }^{14}$ Thus the spread of momentum increases linearly with time. 
In the above example, we assumed that the incident atomic beam has sharp momentum $p=0$, i.e. the incident beam is a plane wave, and hence has infinite transverse extension. For a finite collimated beam of width $\Delta x$, the initial spread of momentum is $\Delta p \sim \hbar / \Delta x$, and the ratio of this spread to the momentum delivered by a single photon is $\Delta p / \hbar k \sim \lambda / 2 \pi \Delta x$. It follows that there is little or no overlap of the terms in Equation (20) when $\Delta x$ is large compared to the optical wavelength, and the probability density for momentum becomes

$$
W(p, t)=\sum_{n=-\infty}^{\infty} j_{n}^{2}(\Omega t) W^{\circ}(p-n \hbar k)
$$

where $W^{\circ}(p)$ is the initial momentum density. The pattern of deflections, in this case, is the same in all essential details as that discussed above.

If the atomic beam diverges with half-angle of say $6=10^{-3}$ rad, and has a typical thermal velocity $v_{z} \sim 5 \times 10^{4} \mathrm{~cm} / \mathrm{sec}$, then the initiai spread of transverse momentum is not small compared with $\hbar_{k}$, and it is expected that interference due to overlap of the terms in Equation (20) will effect the probability density $W(p, t)$. It turns out, however, that, due to a rapidly varying phase factor associated with divergence of the beam, the scale of such interference is small compared to $\Delta p=\hbar k$, and therefore is almost certainly unobservable. We do not present this calculation because the smoothed distribution is the same as Equation (25).

It is interesting to note that Equation (21) is formally identical to the equation for Fraunhofer diffraction of a plane wave by a sinusoidal phase grating. ${ }^{15}$ In effect, the atomic beam is diffracted by the periodic amplitude $(E(x) \propto \cos k x)$ of the standing wave, and the deflection angles, 
$\theta_{n}=n \hbar k / p_{z}$, are precisely what one would expect on the basis of the optical analogy, if the atomic beam is regarded as a wave of wavelength equal to the deBroglie wavelength, $\lambda_{a}=h / p_{z}$.

\section{NUMERICAL EXAMPLE}

Our theory is based on the approximation that recoil and Doppler frequency shifts, $\delta=\hbar \omega^{2} / 2 M c^{2}$ and \pm wp/Mc respectively, are negligible. Accordingly, exponential factors of the form $\exp \{-i[\delta \pm \omega p / M c] t\}$ were replaced by unity in Equation (9). This approximation is valid when $\left(\delta+w|p| / M_{c}\right) t \ll 1$. The recoil shift is half thr Doppler shift when $|p|=\hbar_{k}$. Since we are only interested in cases where $\left.|p| \gg\right\rangle$, the above condition becomes $\omega|p| t / M c \ll 1$. Replacing $|p|$ by the RMS value $\left[\left\langle p^{2}\right\rangle\right]^{1 / 2}=\hbar_{\omega \Omega} t / \sqrt{2} c$ derived above, we obtain a constraint on the interaction time

$$
t<\left[2^{1 / 2} M c^{2} / \hbar \omega^{2} \Omega\right]^{1 / 2} .
$$

The maximum interaction time permitted by Equation (26), $t_{\text {max }}$, determines the thickness of the interaction region, $L=v_{z} t_{\max }$, and the number of absorption-emission processes experienced by the atom, $n=\Omega t_{\max }$. If the atoms issue from an oven at temperature $T, p_{z} \approx \sqrt{2 M k T}$ and $u_{z} \approx \sqrt{2 \mathrm{kT} / M}$ (here $k$ is Boltzmann's constant). The RMS deflection is

$$
\begin{aligned}
{ }_{\text {RMS }} & =\hbar_{\omega \Omega t_{\max }} / 2^{1 / 2} \mathrm{cp}_{\mathrm{z}} \\
& =\left[\hbar_{\Omega / 2} 2^{3 / 2} \mathrm{kT}\right]^{1 / 2}
\end{aligned}
$$


Consider a mildly refractory, moderately massive atom with a strong visible absorption. Let

$$
\begin{aligned}
& T=1000 \mathrm{~K} \\
& M=3.6 \times 10^{-22} \mathrm{~g} \\
& \mu=\langle-|\vec{\mu} \cdot \hat{\varepsilon}|+>=4 \text { Debye } \\
& \omega=3 \times 10^{15} \mathrm{~s}^{-1}
\end{aligned}
$$

Then, for $I=2.5 \times 10^{6}$ watt $/ \mathrm{cm}^{2}$ in the interaction region, we calculate

$$
\begin{aligned}
\Omega & =5.6 \times 10^{11} \mathrm{~s}^{-1} \\
n & =3500 \\
t_{\max } & =6.2 \times 10^{-9} \mathrm{~s} \\
L & =2.6 \times 10^{-4} \mathrm{~cm} \\
\theta_{\text {RMS }} & =3.9 \times 10^{-2} \mathrm{rad}=2.2^{0}
\end{aligned}
$$

Thus a two degree deflection is obtained for our "typical" atom in a field of $2.5 \times 10^{6}$ watt $/ \mathrm{cm}^{2}$. The interaction time required for this deflection is less than the natural lifetime of the transition $\left(\tau \approx 5 \times 10^{-8} \mathrm{~s}\right)$. To achieve the corresponding 2.6 micron beam thickness requires focusing a $2.6 \mathrm{~mm}$ diameter laser beam in one dimension by a factor of $10^{3}$. Thus, for an unfocused laser intensity of $2.5 \mathrm{kw} / \mathrm{cm}^{2}, a$ total laser power of about 130 watts is required. 
Chapter 3

THEORY OF ATOMIC MOTION IN A RESONANT ELECTROMAGNETIC WAVE 
Theory of Atomic Motion in a

Resonant Electromagnetic Wave

\author{
R. J. Cook \\ University of California \\ Lawrence Livermore Laboratory \\ P. 0 . Box 808 \\ Livermore, California 94550
}

\begin{abstract}
A new theory of atomic motion in a resonant standing or traveling electromagnetic wave is presented. It is shown that, when effects of spontaneous emission are negligible, the motion of a two-level atom in the resonant radiation is determined by two noninterfering wave functions, each of which satisfies a time-dependent Schrödinger equation with timeindependent potential energy. An experiment is proposed to test the theory.
\end{abstract}


There has been renewed interest in recent years in the theory of atomic motion ir resonant and near resonant electromagnetic waves. This interest was initiated by the development of high-power tunable lasers, and interest has continued because of possible application of the theory to problems of laser isotope separation $4,10,16$, atomic trapping and cooling $^{17-20}$, neutral atom acceleration ${ }^{21,22}$, and atomic beam deflection spectroscopy $2,5,11$.

An atom illuminated by resonant radiation experiences at least two types of radiation force: a force associated with spontaneous emission, and a force due to interaction of the induced atomic dipole moment with the amplitude gradient of the applied field. The radiation-pressure associated with spontaneous emission has been extensively investigated both theoretically 17,19 and experinental1 $y^{2,3,10}$, and wil7 not be discussed in this letter. A theory of the induced-dipole force has been developed by Ashkin for the case where the atomic resfonse may be described by a polarizability ${ }^{19}$, and a certain off-resonance focussing effect associated with this force has recently been detected experimentally 23 . A description of the atomic response in terms of a polarizability also involves spontaneous emission to the extent that it is spontaneous decay that causes atomic relaxation to the near steady-state condition described by a polarizability. If the atom field interaction is brief (less than a natural lifetime), a theory based on the steady-state polarizability is no Tonger appropriate, and, in general, Schrödinger's equation must be solved to determine the atomic resporse. In this case, effects of spontaneous emission are negligible. 
The purpose of this letter is (1) to present a new theory of atomic motion in a resonant electromagnetic wave, applicable when effects of spontaneous emission are negligible, and (2) to propose an experimental test of the new theory.

The Hamiltonian for an atom in a classically prescribed electromagnetic field, in the dipole approximation, takes the form

$$
H=P^{2} / 2 M+H_{0}-\underline{\mu} \cdot \underline{E}(\underline{R}, t) \text {, }
$$

where $\mathrm{p}^{2} / 2 \mathrm{M}$ is the kinetic energy associated with the center-of-mass momentum $\underline{P}, H_{0}$ is the Hamiltonian for the internal motion of the atom, $\underline{\mu}$ is the dipole moment operator, and $\underline{E}(\underline{R}, t)$ is the electric field evaluated at the center-of-mass position $\underline{R}$. Consider first the motion of a two-level atom with energy leveis $E_{1}$ and $E_{2}$ in a monochromatic standing wave $\underline{E}(\underline{x}, t)=\underline{E} E(\underline{x}) \cos u t$. Here the amplitude $E(\underline{x})$ will be a solution of the time-independent wave equation $\nabla^{2} E+(\omega / c)^{2} E=0$, but is otherwise arbitrary. Let $\psi_{1}(\underline{x})$ and $\psi_{2}(\underline{x})$ be the amplitudes that the atom is located at position $\underline{x}$ and occupies energy levels $E_{7}$ and $E_{2}$ respectively. Then it follows from Eq. (1) that the Schrödinger equation for the two-component wave function is

$$
\begin{aligned}
& \text { ih } \frac{\partial \psi_{1}}{\partial t}=-\frac{-1^{2}}{2 M} \nabla^{2} \psi_{T}+\dot{E}_{1} \psi_{1}-\mu E(\underline{x}) \cos \omega t \psi_{2}, \\
& \text { ith } \frac{\partial \psi_{2}}{\partial t}=-\frac{\hbar^{2}}{2 M} \nabla^{2} \psi_{2}+E_{2} \psi_{2}-\mu E(\underline{x}) \cos \omega i \psi_{1},
\end{aligned}
$$


where $\mathfrak{u}=\langle 1|\underline{\mu} \cdot \underline{\hat{\varepsilon}}| 2\rangle$ is the transition dipole moment. In the case of exact resonance, $\omega=\left(E_{2}-E_{1}\right) / h$, the substitution $\psi_{1}=C_{1} \exp \left\{-i E_{1} t / \pi\right\}, \psi_{2}=$ $C_{2} \exp \left\{-i E_{2} t / \hbar\right\}$ and a rotating wave approximation, i.e., neglect of inessential terms that oscillate at twice the optical frequency, put Eqs. (2) in the form

$$
\begin{aligned}
& \text { in } \frac{\partial c_{1}}{\partial t}=-\frac{\hbar^{2}}{2 ! M} \nabla^{2} c_{1}-\frac{1}{2} l E(\underline{x}) c_{2}, \\
& \text { it } \frac{\partial c_{2}}{\partial t}=-\frac{\hbar^{2}}{2 M} \nabla^{2} C_{2}-\frac{1}{2} \mu E(\underline{x}) C_{1} .
\end{aligned}
$$

Equations (3) are decoupled by the unitary transformation

$$
\begin{aligned}
& u_{+}=2^{-\frac{1}{2}}\left(c_{1}-c_{2}\right), \\
& u_{-}=2^{-\frac{1}{2}}\left(c_{1}+c_{2}\right) .
\end{aligned}
$$

The equations of motion for $u_{ \pm}(\underline{x})$ are

$$
i \hbar \frac{\partial u_{ \pm}}{\partial t}=-\frac{\hbar^{2}}{2 M} \nabla^{2} u_{ \pm} \pm \frac{1}{2} \mu E(\underline{x}) u_{ \pm} \text {, }
$$

and the probability density for the position of the atom, $P(\underline{x})=$

$$
\begin{aligned}
\left|w_{1}(\underline{x})\right|^{2}+\left|\psi_{2}(\underline{x})\right|^{2} & \text {, becomes } \\
& P(\underline{x})=\left|u_{+}(\underline{x})\right|^{2}+\left|u_{-}(\underline{x})\right|^{2} .
\end{aligned}
$$

Equations (5) state that the wave functions $u_{ \pm}$propagate independent of one another, and Eq. (6) shows that there is no spacial interference 
between these waves. An atom initially in the ground state $\left(C_{2}=0\right)$ has equal probability to be in one or the other of the waves $u_{ \pm}$, and Eqs. (5) imply that these probabilities are time-independent. The waves $u_{ \pm}$each satisfy a simple time-dependent Schrödinger equation, but with potential energies $V_{ \pm}(\underline{x})= \pm \frac{1}{2} \mu E(\underline{x})$ of opposite sign. Therefore the forces acting on atoms in the two waves are in opposite directions, and it follows that the amplitude gradient of the resonant radiation will split a narrow atomic beam into two components, in much the same way as the magnetic field gradient splits an atomic beam in the Stern-Gerlach experiment. Equations (5) and (6) are consistent with the theory of Kazantsev that predicts splitting of an atomic trajectory upon crossing a sharp boundary between vacuum and resonant field ${ }^{9}$.

If $\mathrm{N}$ atomic levels take part in the resonant interaction, an analysis similar to the above shows that the motion of the atom is determined by $N$ independent noninterfering wave functions $u_{n}$. The wave function $u_{n}$ satisfies a time-dependent Schrödinger equation with potential energy $v_{n}(\underline{x})=\frac{1}{2} \mu_{n} E(\underline{x})$, where $\mu_{n}$ is the nth eigenvalue of the matrix of transition dipole moments connecting the $N$ levels. The probability density for the position of the atom is $P(\underline{x})=\sum_{n}\left|u_{n}(\underline{x})\right|^{2}$, and the probability that the atom occupies the wave $u_{n}$, for an atom initially in the ground state, is the absolute square of the first element of the $n$th eigenvector of the dipole moment matrix.

If instead of the standing wave $\vec{E}(\vec{x}, t)=\hat{\varepsilon} E(\vec{x})$ cos $\omega t$ we consider a general monochromatic field

$$
\vec{E}(\vec{x}, t)=\varepsilon E(\vec{x}) \cos \{\theta(\vec{x})+\omega t\},
$$


$34 a$

the Schrödinger equation for the motion of a two-level atom becomes

$$
\begin{aligned}
& \text { in } \frac{\partial \psi_{1}}{\partial t}=-\frac{\hbar^{2}}{2 M} \nabla^{2} \psi_{1}+E_{1} \psi_{1}-\mu E(\vec{x}) \cos \left\{\theta(\vec{x})+\omega t \psi_{2},\right. \\
& \text { in } \frac{\partial \psi_{2}}{\partial t}=-\frac{\hbar^{2}}{2 M} \nabla^{2} \psi_{2}+E_{2} \psi_{2}-\mu E(\vec{x}) \cos \{\theta(\vec{x})+\omega t\} \psi_{1} .
\end{aligned}
$$

(8)

Let

$$
\begin{aligned}
& \psi_{1}(\vec{x})=D_{1}(\vec{x}) \exp \left\{-i E_{1} t / \hbar+\frac{1}{2} i[\Delta t+\theta(\vec{x})]\right\}, \\
& \psi_{2}(\vec{x})=D_{2}(\vec{x}) \exp \left\{-i E_{2} t / \hbar-\frac{1}{2} i[\Delta t+\theta(\vec{x})]\right\},
\end{aligned}
$$

where $\Delta=\omega-\omega_{0}$ is the detuning, and $\omega_{0}=\left(E_{2}-E_{1}\right) / \hbar$. Upon substituting (9) into (8), we get

$$
\begin{aligned}
i \hbar \frac{\partial D_{1}}{\partial t}= & -\frac{\hbar^{2}}{2 M}\left(\nabla+\frac{i}{2} \nabla \theta\right)^{2} D_{1}+\frac{1}{2} \hbar \Delta D_{1} \\
& -\mu E \cos \{\theta+\omega t\} D_{2} \mathrm{e}^{-i(\theta+\omega t),} \\
\text { it } \frac{\partial D_{2}}{\partial t}= & -\frac{\hbar^{2}}{2 M}\left(\nabla-\frac{i}{2} \nabla \theta\right)^{2} D_{2}-\frac{1}{2} i \Delta D_{2} \\
& -\mu E \cos \{\theta+\omega t\} D_{1} \mathrm{e}^{i(\theta+\omega t)} .
\end{aligned}
$$

These are exact. Now expand $\cos \{\theta+\omega t\}$ in exponential and keep only slowly varying terms on the right in $(10)$, to obtain

$$
\text { in } \begin{aligned}
& \frac{\partial D_{1}}{\partial t}=-\frac{\hbar^{2}}{2 M}\left(\nabla+\frac{j}{2} \nabla \theta\right)^{2} D_{1}+\frac{1}{2} \hbar \Delta D_{1}-\frac{1}{2} \mu E D_{2}, \\
& \text { in } \frac{\partial D_{2}}{\partial t}=-\frac{\hbar^{2}}{2 M}\left(\nabla-\frac{i}{2} \nabla \theta\right)^{2} D_{2}-\frac{1}{2} \hbar \Delta D_{2}-\frac{1}{2} E D_{1} .
\end{aligned}
$$


As an example, consider the collimated Gaussian beam

$$
\vec{E}(\vec{x}, t)=\hat{\varepsilon} E_{0} \exp \left\{-\left(x^{2}+z^{2}\right) / w_{0}^{2}\right\} \cos \{K y-\omega t\} .
$$

Here we have $E(\vec{x})=E_{0} \exp \left[-\left(x^{2}+z^{2}\right) / w_{0}^{2}\right\}$ and $\theta(\vec{x})=-K y$, and so (11) becomes

$$
\begin{aligned}
\text { it } \frac{\partial D_{1}}{\partial t}= & -\frac{\hbar^{2}}{2 M}\left(\nabla^{2} D_{1}-i K \frac{\partial D_{1}}{\partial y}-\frac{i}{4} K^{2} D_{1}\right) \\
& +\frac{1}{2} \hbar \Delta D_{1}-\frac{1}{2} \mu E(\vec{x}) D_{2}, \\
\text { ik } \frac{\partial D_{2}}{\partial t}= & -\frac{\hbar^{2}}{2 M}\left(\nabla^{2} D_{2}+i k \frac{\partial D_{2}}{\partial y}-\frac{1}{4} K^{2} D_{2}\right) \\
& -\frac{1}{2} \hbar \Delta D_{2}-\frac{1}{2} H E(\vec{x}) D_{1} .
\end{aligned}
$$

To get rid of the trivial terms in $k^{2}$, let $D_{1}=C_{1} \exp \left\{-i \hbar k^{2} t / 8 M\right\}$ and $D_{2}=C_{2} \exp \left\{-i \hbar K^{2} t / 8 M\right\}$. Then

$$
\begin{aligned}
& i \hbar \frac{\partial C_{1}}{\partial t}=-\frac{\hbar^{2}}{2 M} \nabla{ }^{2} C_{1}-\frac{1}{2} \mu E(\vec{x}) C_{2}-\frac{1}{2} \hbar\left[\frac{K}{M}\left(-i h \frac{\partial}{\partial y}\right)-\Delta\right] C_{1} \\
& i \hbar \frac{\partial C_{2}}{\partial t}=-\frac{\hbar^{2}}{2 M} \nabla^{2} C_{2}-\frac{1}{2} \mu E(\vec{x}) C_{1}+\frac{1}{2} \hbar\left[\frac{K}{M}\left(-i \hbar \frac{\partial}{\partial y}\right)-\Delta\right] C_{2}
\end{aligned}
$$

The last terms in (14) and (15) contain factors which in the momentum representation take the form

$$
\frac{K P_{y}}{M}-\Delta .
$$


Here $K P_{y} / M=K v_{y}=\omega v_{y} / c$ is the Doppler shift associated with the velocity $v_{y}$ in the traveling wave (12). If the atomic beam is well collimated (sharp $P_{y}$ ) the Doppler term can always be canceled by an appropriate detuning $\Delta$, and for this detuning the final terms in (14) and (15) may be neglected.

On the other hand, if $c_{1}(\vec{x})$ and $C_{2}(\vec{x})$ are initially independent of $y$ (as in the following example), then they will, according to (14) and (15), remain independent of y for all time, and so, for $\Delta=0$, Eqs. (14) and (15) have solutions satisfying the standing-wave equations (3).

The theory may be tested as follows. Consider a well collimated atomic beam of ground-state two-level atoms that propagates in the $z$ direction and intersects a collimated Gaussian optical beam $E(\underline{x})=$ $E_{0} \exp \left\{-\left(x^{2}+z^{2}\right) / w_{0}^{2}\right\}$ propagating in the $y$ direction. The resonant radiation acts as a cylindrical lens that tends to focus atoms in the wave $u_{-}$and defocus atoms in the wave $u_{+}$, as illustrated in figures 3 and 4 respectively. In crossing the Gaussian beam, the initial plane waves $u_{ \pm}=\exp \{i k z-i \cup i\}$ acquire phase factors $\exp \{\mp i \odot(x)\}$, where $O(x)=\sqrt{\pi} W_{0}{ }^{\mu} E_{0} \exp \left\{-x^{2} N_{0}^{2}\right\} / 2 u_{z}$, and subsequently propagate as freeparticle wave functions. Let $\xi=x / w_{0}, s=z / f_{0}$, and $m=\sqrt{\pi} w_{0} \mu E_{0} / 2 \hbar v z$, where $v_{z}$ is the atomic velocity, $f_{0}=2 W_{0} \varepsilon / \sqrt{\pi} \mu E_{0}\left(\varepsilon=\frac{1}{2} M u_{z}^{2}\right)$ is the "focal length" of the Gaussian lens, and $m$ is a dimensionless measure of the strength of the resonant field. Then the waves $u_{ \pm}$on the down-stream side of the radiation (neglecting inessential phase factors) are given by

$$
u_{ \pm}(\xi, s)=(m / \pi s)^{\frac{1}{2}} \int_{-\infty}^{\infty} \exp \left\{i m\left[s^{-1}\left(\xi-\xi_{0}\right\}^{2}+\exp \left\{-\xi_{0}^{2}\right)\right]\right\} d \xi_{0}
$$


Equation (16) is the result of a Fresnel approximation and is valid when atomic deflections are smal1. To the same approximation, the atomic flux associated with $u_{+}$is $\left.J_{+}(\xi, s)=v_{z} \mid u_{+} \xi, s\right)\left.\right|^{2}$, the flux associated with $u_{-}$is $J_{-}(\xi, s)=v_{z}\left|u_{-}(\xi, s)\right|^{2}$, and the total flux is $J=J_{+}+J_{-}$.

To be specific, consider a IW Gaussian laser beam focussed to waist $W_{0}=50 \mu \mathrm{m}$. The peak intensity is then $I_{0}=2.5 \times 10^{4} \mathrm{~W} / \mathrm{cm}^{2}$ and $E_{0}=$ $\left[8 \pi I_{0} / c\right]^{\frac{1}{2}}$. Let the atomic beam issue from an oven at temperature $T=1000 \mathrm{~K}$, and select velocity component $v_{z}$ equal to $1 / 10$ th of the most probable thermal velocity. Then for "typical" atoms of mass $M=50 u=$ $8 \times 10^{-23} \mathrm{~g}$ and transition dipole moment $\mu=1$ Debye, the focal length of the radiation lens is $f_{0} \approx 0.8 \mathrm{~cm}$, and $\mathrm{m}=7.2 \times 10^{3}$.

The atomic fluxes $J_{-}(\xi)$ and $J_{+}(\xi)$ in the focal plane $(s=1)$ are plotted in figures 5 and 6 respectively. The dominant feature of $J_{-}(\xi)$ is the sharp peak at $\xi=0$. This is the primary focal line of the radiation lens. The flux $\mathrm{J}_{+}(\xi)$ shows two peaks, formed by atomic trajectories that are repelled by the resonant field. The total flux $\mathrm{J}=\mathrm{J}_{+}+\mathrm{J}_{-}$, figure 7 , shows quite clearly the effects of both focussing and defocussing of atomic trajectories. As $s$ increases beyond $s=1$, the peaks of $J_{+}(\xi)$ become sharper and form two secondary focal lines in the plane $s \approx 2.3$ at $\xi \approx \pm 7.8$. These focal lines are a result of focussing by the wings of the negative Gaussian radiation lens. As $s$ varies from 1 to 2.3 the central focal 1 ine of $J_{-}(\xi)$ spreads into a band of width $\Delta \xi \approx 1.0$. The focal lines of $J_{+}(\xi)$ lie well outside of this band. The structure of the atomic flux would be well resolved by a detector of resolution $\Delta x=10 \mu \mathrm{m}$. 
A direct measurement of the splitting of an atomic beam by the amplitude gradient of the resonant field may be accomplished by placing a narrow slit immediately upstream of the interaction region, as illustrated in figure 8 . The maximum splitting occurs when the center of the slit is off-axis by the amount $\delta=w_{0} / \sqrt{2}$, and, in the above example, has the value $\theta=0.31^{\circ}$. For a siit width of $20 \mu \mathrm{m}$, the divergence of each of the deflected components is less than $0.03^{\circ}$

The present theory is valid when effects of spontaneous emission are negligible, i.e., when the interaction time is less than the natural lifetime of the atoms $\left(\Delta t=2 W_{0} / v_{z}<\tau_{n}\right)$. In the above example, this condition obtains when the resonant frequency is less than $2.5 \times 10^{15} \mathrm{~Hz}$ or $\lambda \gtrsim 0.7 \mathrm{~m}$. This constraint may be relaxed somewhat by decreasing $\mu$, $M$ and/or $W_{0}$.

A measurement of the total atomic flux in the focal plane $(s=1)$ showing the triple-peaked structure of $J(\xi)$, or a direct measurement of the splitting of an atomic beam by the amplitude gradient of the resonant field, would provide a convincing test of the present twocomponent theory. 
Chapter 4

DIFFRACTION OF ATOMS AND MOLECULES BY

A RESONANT STANDING LIGHT WAVE 
Diffraction of Atoms and Molecules by a Resonant Standing Light Wave

\author{
R. J. Cook \\ University of California \\ Lawrence Livermore Laboratory \\ P.0. Box 5508 \\ Livermore, California 94550
}

\title{
Abstract
}

Diffraction of atoms and molecules by a strong resonant standing electromagnetic wave is investigated theoretically. Previous work is here generalized to include the case in which several atomic or molecular energy levels interact strongly with the resonant radiation. It is shown that, when effects of spontaneous emission are negligible and the radiation is tuned to exact resonance, the motion of the atom or molecule is determined by a set of independent noninterfering eigenwaves, each of which satisfies a time-dependent Schrödinger equation with time-independent potential energy. The structure of the eigenwaves and associated potential energies is investigated for (1) a two-level atom, (2) a two-level atom with degenerate upper level, (3) a truncated harmonic oscillator, and (4) an ideal simple harmonic oscillator. An equation describing propagation of a paraxial atomic or molecular beam in a resonant standing wave is derived, and an exact solution is given in terms of Mathieu functions. The physical significance of this solution is discussed in connection with the Raman-Nath equation, and it is pointed out that the problem of diffraction of atoms or molecules by a resonant standing light wave is formally anaiogus to the problem of diffraction of light by ultrasound. 


\section{INTRODUCTION}

In a recent letter ${ }^{24}$ (hereafter referred to as I) it was shown that, when effects of spontaneous emission are negligible, the motion of a two-leve1 atom in a resonant standing electromagnetic wave is determined by two independent noninterfering wave functions, $u_{+}(\vec{x})$ and $u_{-}(\vec{x})$, each of which satisfies a time-dependent Schrödinger equation but with potential energies $V_{ \pm}(\vec{x})= \pm \frac{1}{2} \mu E(x)$ of opposite sign (here $\mu$ is the transition dipole moment between the two leve]s and $E\left[y_{;}\right.$is the amp $\}$itude of the standing wave). Initially, when the atom is in its ground state, the two waves $u_{ \pm}(\vec{x})$ are identical, but because the potential gradients acting on the waves are in opposite directions a small atomic wave packet is split by the resonant radiation. An experiment designed to detect this splitting in laser radiation was proposed in I. Similar theoretical results and observations in the microwave region have been reported by Kazantsev ${ }^{25}$, Hill and Gallagher ${ }^{26}$, and Bloom et. a1. ${ }^{27}$

In a related work by Cook and Bernhardt ${ }^{28}$ (hereafter referred to as II) diffraction of a beam of two-level atoms by a resonant standing wave was investigated in the limit of short atom-field interaction time. It was found, in this limit, that the probability $P_{n}$ that the atom aquires transverse momentum $n \hbar \omega / c$ (the momentum of $n$ photons) while moving parallel to the fringes of a plane standing wave is given by the relation $P_{n}(t)=J^{2}(\Omega t)$, where $J_{n}$ is the Bessel function of order $n, \Omega$ is the Rabi flopping frequency of the two-level atom, and $t$ is the interaction time. 
The purpose of the present paper is to generalize the above work in two directions. First, the theory of atomic motion in a resonant standing wave is extended to include the case in which more than two atomic (or molecular) energy levels interact strongly with the resonant radiation, and second, the constraint of short atom-field interaction time in II is removed by showing that an exact solution to the diffraction problem can be written in terms of Mathieu functions. The intent of this generalization is to provide a theory of sufficient generality to make possible more realistic comparison with experiment. In the following we shall use the term "atom" to refer to either an atom or a molecule.

In the model considered here the internal and translational motions of the atom are treated quantum-mechanically while the field is treated classically. Our theory treats only the case of exact resonance, and spontaneous emission is ignored. Thus the present theory is expected to give a valid description of atomic motion in a strong resonant standing wave for interaction time less than the shortest natural lifetime of the several interacting levels.

In the following section the basic theory of atomic motion in a resonant standing wave is developed. It is shown that, if $\mathrm{N}$ levels take part in the resonant interaction, the motion of the atom is determined by N independent noninterfering "eigenwaves", each of which satisfies a simple one-component Schrödinger equation with time-independent potential energy. In Section III the structure of the eigenwaves and associated 
eigenwaves and associated potential energies is studied for four cases likely to be encountered in practice: (1) a two-level atom, (2) a two-level atom with degenerate upper level, (3) a truncated harmonic oscillator, and (4) an ideal simple harmonic oscillator. Section IV contains a derivation of the differential equation describing propagation of a paraxial atomic beam in a resonant standing wave, and an exact solution to this equation is derived and discussed in Section $v$.

\section{BASIC THEORY}

As in previous work (I and II) we start with the Hamiltonian for an atom in a classically prescribed electromagnetic field in the electric dipole approximation,

$$
H=P^{2} / 2 M+H_{0}-\vec{\mu} \cdot \vec{E}(\vec{R}, t)
$$

where $P^{2} / 2 M$ is the kinetic energy associated with the center-of-mass momentum $\overrightarrow{\mathrm{P}}, H_{0}$ is the Hamiltonian for the internal motion of the unperturbed aton, $\vec{H}$ is the electric dipole moment operator, and $\vec{E}(\vec{R}, t)$ is the electric field evaluated at the center-of-mass position $\vec{R}$. We consider the motion of an atom in a monochromatic standing wave

$$
\vec{E}(\vec{x}, t)=\hat{E} E(\vec{x}) \cos \omega t
$$

where $\hat{\varepsilon}$ is a unit polarization vector independent of position and time. 
In the position representation for the center-of-mass motion and the energy representation for the internal motion, the basis states are $|n, \vec{x}\rangle=|n>| \vec{x}\rangle$, where $\mid n>$ is an eigenvector of $H_{0}\left(H_{0}\left|n>=E_{n}\right| n>\right)$, and $\mid \vec{x}>$ is an eigenvector of the center-of-mass position operator $\vec{R}$ with eigenvalue $\vec{x}(\vec{R}|\vec{x}>=\vec{x}| \vec{x}>)$. An arbitrary state vector is expanded as

$$
\left.\left|\psi>=\sum_{n} \int d^{3} x c_{n}(\vec{x}) e^{-i E_{n} t / h}\right| n, \vec{x}\right\rangle
$$

where $c_{n}(\vec{x})$ is the amplitude for position $\vec{x}$ and internal energy $E_{n}$. Inserting this expansion into the Schrödinger equation, iha $|\psi\rangle / a t=H|\psi\rangle$, making use of the orthonormality of the basis states $|n, \vec{x}\rangle$, and evaluating the matrix elements of the Hamiltonian, Equation (1), with $\vec{E}(\vec{R}, t)$ taken from Equation (2), we obtain the equations of motion

$$
\begin{aligned}
& i \hbar \frac{\partial c_{n}(\vec{x})}{\partial t}=-\frac{h^{2}}{2 M} \nabla^{2} c_{n}(\vec{x}) \\
& -E(\vec{x}) \sum_{m} \mu_{n m^{\prime} m} c_{m}(\vec{x}) \cos (\omega t) e^{i \omega n m^{t}}
\end{aligned}
$$

where $\mu_{n m}=\langle n|\vec{\mu} \cdot \hat{\varepsilon}| m\rangle$ and $\omega_{n m}=\left(E_{n}-E_{m}\right) / n$.

The following calculation is limited to the case in which the applied field frequency $\omega$ is equal to one of the resonant frequencies of the atom. In general several atomic transitions are simultaneously in resonance with the applied field. This occurs, for example, when the field induces transitions between two energy levels each of which is 
degenerate, or when the atom (molecule) is an harmonic oscillator, in which case the entire ladder of transitions is in resonance with the field. The states $n$ and $m$ strongly affected by the field are those that satisfy $\left|\omega_{n m}\right|=\omega$.

Next a rotating wave approximation is introduced by expanding $\cos$ ( $\omega t$ ) in exponentials and keeping only terms on the right in Equation (4) that vary slowly with time. The result is

$$
\begin{aligned}
& i \hbar \frac{\partial c_{n}(\vec{x})}{\partial t}=-\frac{\hbar^{2}}{2 M} \nabla^{2} c_{n}(\vec{x}) \\
& -\frac{1}{2} E(\vec{x}) \sum_{m} \tilde{\mu}_{n m} c_{m}(\vec{x})
\end{aligned}
$$

where $\tilde{\mu}_{\mathrm{nm}}=\mu_{\mathrm{nm}}$ for $\left|\omega_{\mathrm{nm}}\right|=\omega$, and $\tilde{\mu}_{\mathrm{nm}}=0$ otherwise. The indesies $n$ and $m$ in Equations (5) range over the entire set of atomic states. It is convonient to partition this set into (1) the set of $\mathbf{N}$ states that interact with the field (essential states), and (2) the remaining set of states that do not interact with the field (inesscntial states). With a rearrangement of state lables, the amplitudes $c_{n}(\vec{x})$ for essential states satisfy Equations (5) with $n$ and $m$ running from $l$ to $N$, and $\tilde{u}_{n m}$ becomes an $N \times N$ matrix. The amplitudes for inessential states satisfy free particle Schrödinger equations, ina $c_{n} / \partial t=$ - $\left(\hbar^{2} / 2 M\right) \nabla^{2} c_{n}$. We shall assume that the atom starts out in its ground state, and that the ground state is an essential state. In this case, the inessential states may be ignored. 
The coupled set of differential equations for essential states, Equations (5), is decoupled by a unitary transformation. Let

$$
c_{n}(\vec{x})=\sum_{k=1}^{N} u_{n k} u_{k}(\vec{x})
$$

Where $U_{n k}$ is the unitary matrix that diagonalizes $\tilde{\mu}_{n m}$,

$$
\sum_{i, j=1}^{N} U_{n j}^{+} \tilde{\mu}_{j k} u_{k m}=\mu_{n}{ }^{5} \text {. }
$$

$\mu_{n}$ are the eigenvalues of $\tilde{\mu}_{n m}$. Upon substituting (6) into (5), matrix multiplying the result on the left by $U_{L n}^{-1}=U_{L n}^{+}$, and using Equation (7), we obtain the decoupled equations

$$
i \hbar \frac{\partial u_{n}(\vec{x})}{\partial t}=-\frac{\hbar^{2}}{2 M} \nabla^{2} u_{n}(\vec{x})-\frac{1}{2} u_{n} E(\vec{x}) u_{n}(\vec{x}) \text {. }
$$

Equation (8) indicates that the "eigenwave" $u_{n}(\vec{x})$ satisfies a singlecomponent Schrödinger equation with potential energy $v_{n}(\vec{x})=-\frac{1}{2} \mu_{n} E(\vec{x})$. The various eigenwaves propagate independently under the influence of their respective potential energies, and it follows from (6) that the probability density for the position of the atom, $P(\vec{x})=\Sigma_{n}\left|c_{n}(\vec{x})\right|^{2}=$ $\Sigma_{n}\left|u_{n}(\vec{x})\right|^{2}$, is the sum of the densities $\left|u_{n}(\vec{x})\right|^{2}$ for each of the eigenwaves, i.e. there is no interference between different eigenwaves. From the inverse of Equation (6),

$$
u_{n}(\vec{x})=\sum_{k=1}^{N} U_{n k}^{+} c_{k}(\vec{x})
$$


we find that if the atom is initially in its ground state $\left(c_{k}(\vec{x}, 0)=0\right.$ for $k>1)$, then

$$
u_{n}(\vec{x}, 0)=u_{l n}^{*} c_{1}(\vec{x}, 0)
$$

and the probability $w_{n}$ that the atom occupies the nth eigenwave is

$$
w_{n}=\int d^{3} x\left|u_{n}(\vec{x}, 0)\right|^{2}=\left|u_{l n}\right|^{2} .
$$

These probabilities are independent of time.

The theory may be illustrated by considering a narrow beam of atoms that traverses a section of standing wave in which $D E$ is transverse to the beam. According to Ehrenfest's theorem, atoms in the nth eigenwave experience a deflecting force $\vec{F}_{n}=-\left\langle\nabla V_{n}\right\rangle=\frac{1}{2} \mu_{n}\langle\nabla E\rangle$ proportional to $\mu_{n}$, and hence, the atomic beam is split into as many components as there are distinct eigenvalues $\mu_{n}$. If the eigenvalues $\mu_{n}$ are nondegenerate, this technique may be used to separate the different eigenwaves, in much the same way as different angular momentum states are separated in the Stern-Geriach experiment.

\section{SPECIFIC EXAMPLES}

In this section the above theory is applied to four specific cases likely to be encountered in practice, and for which diagonalization of $\tilde{u}_{\mathrm{nm}}$ has been found to be analytically tractable. 
A. Simple two-level atom

For an appropriate choice of the phases of $|n\rangle$, the dipole moment matrix takes the form

$$
\tilde{u}_{\mathrm{nm}}=\left[\begin{array}{ll}
0 & \mu \\
\mu & 0
\end{array}\right] \text {, }
$$

where $\mu$ is a real number. The unitary matrix that diagonalizes (12) is

$$
u_{n m}=2^{-1 / 2}\left[\begin{array}{rr}
1 & 1 \\
1 & -1
\end{array}\right] \text {, }
$$

and the eigenvalues are $\mu_{1}=\mu, \mu_{2}=-\mu$. The eigenwaves are related to the amplitudes $c_{1}(\vec{x})$ and $c_{2}(\vec{x})$ of the two-level atom by the equations

$$
\begin{aligned}
& u_{1}(\vec{x})=2^{-1 / 2}\left[c_{1}(\vec{x})+c_{2}(\vec{x})\right], \\
& u_{2}(x)=2^{-1 / 2}\left[c_{1}(\vec{x})-c_{2}(\vec{x})\right],
\end{aligned}
$$

and these eigenwaves propagate independently with potential energies $v_{1}(\vec{x})=-\frac{1}{2} \mu E(\vec{x})$ and $V_{i}(\vec{x})=\frac{1}{2} \mu E(\vec{x})$ respectively. A two-level atom initially in its ground state has equal probability to be in either of the two eigenwaves $\left(W_{1}=W_{2}=1 / 2\right)$, and since the eigenvalues $\mu_{1}$, $\mu_{2}$ are distinct, a narrow beam of two-level atoms splits into two beans when it encounters the amplitude gradient of a standing wave, i.e. a standing wave acts as a birefringent medium for two-level atoms. This simple case was treated previously in I. 
B. Two-leve1 atom with degenerate upper level

Consider a two-level atom with nondegenerate ground state (amplitude $\left.c_{1}(\vec{x})\right)$ and a degenerate upper level consisting of $N$ - 1 states (amplitudes $\left.c_{n}(\vec{x}), n=2, \ldots, N\right)$. Let $\tilde{u}_{n l}$ be the transition dipole moment between the ground state and the upper state of index $n$. The dipole moment matrix takes the form

$$
\tilde{\mu}_{n \mathrm{~m}}=\left[\begin{array}{ccccc}
0 & \tilde{\mu}_{12} & \tilde{\mu}_{13} & \cdots & \tilde{\mu}_{1 N} \\
\tilde{\mu}_{21} & 0 & 0 & \cdots & 0 \\
\tilde{\mu}_{31} & 0 & 0 & \cdots & 0 \\
\vdots & \vdots & \vdots & & \vdots \\
\dot{\mu}_{N 1} & 0 & 0 & \cdots & 0
\end{array}\right]
$$

with $\tilde{\mu}_{j n}=\tilde{\mu}_{n 1}^{\star}$. Let $v_{m}$ be an eigenvector of $\tilde{\mu}_{n m}$ with

eigenvalue $\mu$. The eigenvalue equation, $\Sigma_{m}{ }_{\mu}^{n}{ }_{n m} v_{m}=\mu v_{n}$, becomes

$$
\sum_{m=2}^{N} \stackrel{\sim}{\mu}_{1 m} v_{m}={ }^{v} v_{1}
$$

for $n=1$, and

$$
\tilde{\mu}_{n l} v_{1}=\mu v_{n}
$$

for $n>1$. There exist solutions of these equations with $v_{1}=0, \mu=0$ and $\Sigma_{m} \tilde{\mu}_{m l}^{\star} v_{m}=0$. If $\tilde{\mu}_{m l}(m=2, \ldots, N)$ are regardad as the components of a vector in $\mathrm{N}-1$ dimensions, the last equation states that $v_{m}(m=2, \ldots, N)$ is a vector orthogonal to $\tilde{\mu}_{m l}$. In $N-1$ dimensions 
there are N - 2 such vectors, and hence, there are N - 2 eigenvectors of $\tilde{u}_{n m}$ with $v_{1}=0$ and $\mu=0$. Now the mth column of the unitary matrix $U_{\mathrm{rm}}$ that diagonalizes $\tilde{\mu}_{\mathrm{nm}}$ is the eigenvector of $\tilde{\mu}_{\mathrm{nm}}$ with eigenvalue $u_{m}$. Thus Equation (9) states that $u_{n}(\vec{x})$ is the inner product of the nth eigenvector of $\tilde{\mu}_{n m}$ with $c_{m}(x)$, and (11) tells us that the probability $W_{n}$ is the absolute square of the first component of the $n$th eigenvector of $\tilde{\mu}_{n m}$. Because each of the $N-2$ eigenvectors we have been considering has zero first conponent $\left(v_{1}=0\right)$, the probability is zero that any of the eigenwaves associated with these eigenvectors is occupied.

The remaining two eigenvectors of $\tilde{u}_{\mathrm{nm}}$, or columns of $U_{\mathrm{nm}}$, are obtained by solving Equations (16) and (17) with $\mu \neq 0$. Upon solving (17) for $v_{n}$,

$$
v_{n}=\tilde{u}_{n 1} v_{1} / \mu \text {, }
$$

and inserting this into (16), we get the eigenvalues

$$
\mu_{ \pm}= \pm\left[\sum_{m=2}^{N}\left|\tilde{u}_{\mathrm{m} \mid}\right|^{2}\right]^{1 / 2}= \pm 0
$$

$\sigma$ is the r.m.s. of the transition dipole moments between ground and excited states. The components of the two eigenvectors are obtained from (18) iy nomalization. The result is $v_{l}^{ \pm}=2^{1 / 2}, v_{n}^{ \pm}= \pm 2^{-1 / 2} \tilde{\mu}_{n l} / 0(n=2$, $\ldots, N)$. Finally, the inner product of these vectors with $c_{m}(\vec{x})$ gives a formula for the occupied eigenwaves, 


$$
u_{ \pm}(\vec{x})=\frac{1}{\sqrt{2}}\left[c_{1}(\vec{x}) \pm \frac{1}{\sigma} \sum_{m=2}^{N} \underset{\mu_{1 m}}{\ddot{j}} c_{m}(x)\right] \cdot
$$

The atom occupies these eigenwaves with probabilities $w_{ \pm}=\left|v \frac{ \pm}{1}\right|^{2}=1 / 2$.

C. Truncated harmonic oscillator

The interaction of resonant radiaton with the vibrational levels of a molecule may be approximated by treating the molecule as an $N$ - level system $(n=1,2, \ldots, N)$ with $E_{n}=\hbar_{\omega}(n-1 / 2)$ and transition dipole moments equal to those of a simple harmonic oscillator,

$$
\tilde{\mu}_{n m}=\mu\left[\sqrt{n} \delta_{n+1, m}+\sqrt{n-1} \delta_{n-1, m}\right] .
$$

Here $\mu$ is the moment for the $n=1$ to $n=2$ transition. Truncation of simple harmonic oscillator levels for $n>N$ takes into account, in an approximate way, the fact that higher vibrational levels in real molecules do not interact with the applied field due to anharmanicity.

It was shown by Białynicka-Birula et.ar.$^{30}$ that the eigenvalues of (21) are $\mu_{m}=2^{1 / 2} \mu \lambda_{m}$, where $\lambda_{m}$ are the roots of the ith Uermite polynomial $H_{N}(x)$, and that the eigenvector associated with $\mu_{m}$ has components

$$
v_{n}\left(\mu_{m}\right)=\sqrt{\frac{N ! 2^{N-n}}{(n-1) !}} \frac{H_{n-1}\left(\lambda_{m}\right)}{\frac{N H_{N-1}\left(\lambda_{m}\right)}{N}} \cdot
$$


It then follows from the arguments of the preceeding subsection that the mth eigenwave is

$$
u_{m}(\vec{x})=\sum_{m=1}^{N} v_{n}\left(\mu_{m}\right) c_{n}(\vec{x}),
$$

and the probability that the molecule occupies the mth eigenwave is $w_{m}=\left|v_{1}\left(\mu_{m}\right)\right|^{2}$. The eigenvalues $\mu_{m}$ are distinct. Therefore the amplitude gradient of a standing wave splits a narrow beam of truncated harmonic oscillators into $\mathbf{N}$ distinct components.

\section{Harmonic oscillator}

As a final example consider a molecule whose internal motion is that of an ideal simple harmonic oscillator of effective mass $m$, effective charge $e$, and frequency w. For an ideal harmonic oscillator all of the energy levels are essential, and the matrix $\tilde{\mu}_{n m}$ of transition dipole moments between essential states is the complete matrix of dipole moments. Let $\hat{q}$ be the component of the oscillator amplitude in the direction $\hat{\varepsilon}$ of the applied electric field. The component of the dipole moment in this direction is $\tilde{\mu}=e \hat{q}$, and this operator is manifestly diagonal in the q-representation. Therefore the eigenvectors of $\tilde{\mu}$ are the eigenvectors $\mid q>$ of $\hat{q}$, and the eigenvalues $\mu=$ eq assume all real values. The eigenvectors of $\tilde{\mu}$ in the energy reprepresentation have components $v_{n}(q)=\langle n \mid q\rangle$, where $|n\rangle(n=0,1,2, \ldots)$ are the 
energy eigenstates of the oscillator. As usual, the eigenwaves (here labeled by the continuous index $q$ ) are determined by $v_{n}(q)$ through the relation

$$
u(q, \vec{x})=\sum_{n} v_{n}^{\star}(q) c_{n}(\vec{x})
$$

and the probability (here a density) that the molecule occupies the eigenwave of eigenvalue $\mu=\mathrm{eq}$ is the absolute square of the first component of $v_{n}(q)$, namely, $w(q)=\left|v_{0}(q)\right|^{2}=|<q| 0>\left.\right|^{2}$. From the known form of the ground state wave function of an harmonic oscillator, we find that $w(q)=(m \omega / \pi \hbar)^{1 / 2}$ exp $\left\{-m \omega q^{2} / \hbar\right\}$, and the associated probability density for eigenyalues $\mu=$ eq is

$$
W(\mu)=\left(2 \pi \mu_{0}^{2}\right)^{-1 / 2} \exp \left\{-\mu^{2} / 2 \mu_{0}^{2}\right\}
$$

where $\mu_{0}=e(\hbar / 2 m \omega)^{\frac{1}{2}}$ is the transition dipole moment between oscillator states $n=0$ and $n=1$. The eigenwave $u(q, \vec{x})$ moves in potential energy $v(q, \vec{x})=-\frac{1}{2}$ eqE$(\vec{x})$.

The above results indicate that a narrow beam of ideal harmonic-oscillator molecules is not split into a discrete set of beams by the amplitude gradient of the standing wave, but rather, the beam experiences a gaussian spreading owing to the continuous distribution of eigenvalues $\mu$ and the gaussian occupation probability for eigenwaves $u(q, \vec{x})$. 


\section{PROPAGATION OF PARAXIAL ATOMIC BEAMS}

The differential equation for the eigenwave " $n$ "

$$
\text { in } \frac{\partial u_{n}}{\partial t}=-\frac{n^{2}}{2 M} \nabla^{2} u_{n}-\frac{1}{2} \mu_{n}^{E}(\vec{x}) u_{n} \text {, }
$$

has steady solutions of the form

$$
u_{n}(\vec{x}, t)=\psi_{n}(\vec{x}) \exp \left\{i k z-i \omega_{0} t\right\}
$$

with $h_{0}(1)=(\hbar k)^{2} / 2 M$. When $u_{n}(\vec{x}, t)$ is a paraxial wave propagating in the positive $z$ direction, $\psi_{n}(\vec{x})$ is a slowly varying function of $z$. Inserting (27) into (26), we obtain

$$
2 i k \frac{\partial \psi_{n}}{\partial z}=-\nabla^{2} \psi_{n}-\frac{M u_{n}}{\hbar^{2}} E(\vec{x})_{\psi_{n}} \cdot
$$

On the assumption that $\psi_{n}$ is a slowly varying function of $z$, we may discard the small term $-\partial^{2} \psi_{n} \partial z^{2}$ on the right in (28) which is negligible compared to the term containing $\partial \psi_{n} / \partial z$. In optics this is known as a Fresnel approximation. Equation (28) becomes

$$
2 i k \frac{\partial \psi_{n}}{\partial z}=-\nabla_{T}^{2} \psi_{n}-\frac{M_{u}}{\hbar^{2}} E(\vec{x})_{n}
$$

where $\nabla_{T}^{2}=\partial^{2} / \partial x^{2}+a^{2} / \partial y^{2}$ is the transverse $L a p l a c i a n$. In vacuum $(E=0)$ the solution of (29) is the Fresnel diffraction integral, 


$$
\psi_{n}(\vec{p}, z)=\frac{k}{2 \pi i\left(z-z_{0}\right)} \int \exp \left\{\frac{i k\left|\vec{p}-\vec{p}_{0}\right|^{2}}{2\left(z-z_{0}\right)}\right\} \psi_{n}\left(\vec{\rho}_{0}, z_{0}\right) d^{2} \rho_{0} .
$$

where $\vec{\rho}=(x, y)$.

Let $c_{0}(\vec{p})$ be the ground state wave function at $z=0, t=0$. Then according to $(10)$ and $(27)$ the initial eigenwaves are $\psi_{n}(\vec{p}, 0)=U_{\eta_{n}}^{*} c_{0}(\vec{p})$ But, as noted above, there is no interference between different eigenwaves, and consequently the ritial phase of each eigenwave may be shifted by a constant withou. : inc ing the result. We use this freedom to replace $u_{1 n}^{*}$ by its modulus $i$ it preceeding equation, and write the initial eigenwaves as $\psi_{n}\left(\vec{\rho}, u \quad{ }^{\prime}{ }^{2} c_{0}(\vec{\rho})\right.$

In a typical experiment a beam of ground-state atoms enters the standing wave at $z=0$, exits the wave at $z=L$, and propagates in vacuum to 1 arge positive $z$ where the effect of the interaction is detected by measuring the atomic flux $J(\vec{p}, z)$. The atomic flux, or $z$ component of the probability current density, is calculated in the usual way except that terms containing $\partial \psi_{n} / \partial z$ must be discarded to obtain a result consistent with the Fresnel approximation. The total flux is

$$
J(\vec{p}, z)=(\hbar k / M) \sum_{n=1}^{N}\left|\Psi_{n}(\vec{p}, z)\right|^{2}
$$

Upon exiting the interaction region, the eigenwaves $\psi_{n}(\vec{p}, L)$ may be transformed to the transverse momentum representation 


$$
\phi_{n}(\vec{P}, L)=(2 \pi h)^{-1} \int \psi_{n}(\vec{\rho}, L) e^{-i \vec{p} \cdot \vec{\rho} / h_{d}{ }^{2} \rho}
$$

Then by using the standard interpretation of the momentum representation wave function, or by analogy with Fraunhofer diffraction theory, it is readily shown that as $z \rightarrow \infty$ the atomic flux approaches

$$
J(\vec{n}, z)=\frac{(\hbar k)^{3}}{M z^{2}} \underset{n=1}{N}\left|\phi_{n}(\hbar k \vec{k} / z, L)\right|^{2}
$$

\section{v. DIFFRACTION OF ATOMS BY A SIMPLE STANDING WAVE}

Consider a simple standing wave of amplitude $F(x, z)=2 E_{0} \cos k_{0} x$ for $0 \leqslant z \leqslant L$ and zero otherwise, where $k_{0}=2 \pi / \lambda$ is the propagation constant. Atomic motion in the $y$ direction is unaffected by the field and may therefore be ignored. Equation (28) becomes

$$
2 i k \frac{\partial \psi_{n}}{\partial z}=-\frac{\partial^{2} \psi_{n}}{\partial x^{2}}-\frac{2 M \mu_{n} E_{0}}{\hbar^{2}} \cos k_{0} x \psi_{n}
$$

for $0 \leqslant z \leqslant L$, and $2 i k \partial \psi_{n} / \partial z=-\partial^{2} \psi_{n} / \partial x^{2}$ elsewhere. Equation (34) has solutions of the form $\psi_{n}(x, z)=g(x) f(z)$ with $f(z)=\exp \{-j \lambda z\}$ and

$$
\frac{d^{2} g}{d x^{2}}+2\left(\lambda k+\frac{M \mu_{n} E_{0}}{\hbar^{2}} \cos k_{0} x\right) g=0
$$

where $\lambda$ is a separation constant. The change of variables $k_{0} x=2 \xi$ yields 


$$
\frac{d^{2} g}{d \xi^{2}}+\left(a-2 a_{n} \cos 2 \xi\right) g=0
$$

where $a=8 \lambda k / k_{0}^{2}$ and $q_{n}=-4 M_{n} E_{0} /\left(\hbar k_{0}\right)^{2}$. Equation (36) is Mathieu's equation?

If the incident atomic wave function is a plane wave propagating in the positive $z$ direction $\left(c_{0}(x)=1, \psi_{n}(x, 0)=w_{n}^{1 / 2}\right)$, the solution of (34) will be an even periodic function of $x$. The even periodic solutions of (36) are the Mathieu functions $\operatorname{ce}_{r}\left(\xi, q_{n}\right)$, and the associated characteristic values are $a=a_{r}\left(q_{n}\right)^{31}$. These functions form a complete orthogonal set for the expansion of even functions of period $\Delta E=2 \pi$, and the solution of (34), with initial condition $\psi_{n}(x, 0)=w_{n}^{1 / 2}$, can be expanded in terms of them in the usuat way. The result is

$$
\psi_{n}(x, z)=w_{n}^{1 / 2} \sum_{r=0}^{\infty} d_{r}^{n} c e_{r}\left(\frac{1}{2} k_{0} x, q_{n}\right) e^{-i \lambda} r^{n} z
$$

where $\lambda_{r}^{n}=k_{0}^{2} a_{r}\left(q_{n}\right) / 8 k$, and $d_{r}^{n}=\pi^{-1} \int_{0}^{2 \pi} \operatorname{ce} r\left(\xi, q_{n}\right) d \xi$.

Equation (37) is an exact solution to Equation (34) for $0 \leqslant z \leqslant L$. the solution for $z>L$ is given by Equation (30) with $z_{0}=L$, and, in the the 1 imit $z \rightarrow \infty$, the atomic flux $J(x, z)$ is obtained by evaluating Equations (32) and (33). Thus an exact solution to the diffraction 
problem is easily written down. However, a solution in terms of Mathieu functions is not particularly convenient for numerical evaluation nor does it provide a clear physical picture of the scattering process.

Perhaps a better approach is to assume a solution of the form

$$
\psi_{n}(x, z)=w_{n}^{1 / 2} \sum_{m=-\infty}^{\infty} A_{m}(z) e^{i m k_{0} x}
$$

with $A_{m}(0)=\delta_{m 0}$. The mth term in (39) is the amplitude that the atom is moving with transverse momentum mihk (the momentum of $m$ photons) and the initial condition $A_{m}(0)=\delta_{\text {mo }}$ indicates that the atom starts out with zero transverse momentum. Upon substituting (39) into (34) we get equations for the implitudes $A_{m}$,

$$
i \frac{d A_{m}}{d z}=\frac{m^{2} k_{0}^{2}}{2 k} \quad A_{m}-\frac{M \mu_{n} E_{0}}{2 \hbar^{2} k_{0}}\left(A_{m-1}+A_{m+1}\right) .
$$

The change of variables $z=v_{z} t=(\hbar k / M) t$ from $z$ to interaction time $t$, and a further change of variables $\tau=\Omega_{n} t$ to dimensionless time $\tau$, where $\Omega_{n}=\mu_{n} E_{0} / h$, reduces Equation (40) to

$$
i \frac{d A_{m}}{d t}=a m^{2} A_{m}-\frac{1}{2}\left(A_{m-1}+A_{m+1}\right)
$$

where $\alpha=\left(\hbar k_{0}\right)^{2} / 2 M_{\mu_{n}} E_{0}$ is a dimensionless parameter. The probability that an atom in eigenwave $n$ has aquired transverse momentum 
mrik $_{0}$ after interacting with the radiation for time $t$ is $P_{m}(t)=$ $\left|A_{\pi}\left(\Omega_{n} t\right)\right|^{2}$. Equation (41) applies when $0 \leqslant z \leqslant L$ or $0 \leqslant \tau \leqslant M \mu_{n} E_{d} / h^{2} k_{\text {. }}$ For $z>L, d_{m} / d z=0$.

Equation (41) is one form of the Raman-Nath equation. The Raman-Nath equation has been studied extensively in connection with diffraction of light by ultrasonic waves 32 . Here we see that diffraction of atoms by a resonant standing eltiviomagnetic wave is formally analogus to diffraction of light by ultrasound. This formal analogy allows us to carry over many of the results of the theory of diffraction by ultrasound and apply them directly to the present problem.

Probably the most significant difference between the two theories is that diffraction of atoms by a standing wave involves simultaneous diffraction of $N$ atomic eigenwaves, with $N=2$ as a minimum, while diffraction of light by ultrasound involves only a single light wave. Another difference is the value of the parameter $\alpha$. In diffraction by ultrasound this parameter typically has a value of order $10^{-1}$ or larger, whereas the $\alpha$ for atomic diffraction by a strong standing light wave can easily be as small as $10^{-6}$.

To understand the significance of $t n_{1}$, parameter, consider the case in which $\alpha$ is small compared to unity. Then initially the term $\alpha_{m}{ }^{2} A_{m}$ in Equation (41) may be neglected, and the substitution $A_{m}=(i)^{m} B_{m}$ reduces (41) to the form 


$$
\frac{d B_{m}}{d \tau}=\frac{1}{2}\left(B_{m-1}-B_{m+1}\right) .
$$

This is a recurrence relation for Bessel functions. The solution satisfying initiai conditions $B_{m}(0)=\delta_{m o}$ is $B_{m}(\tau)=J_{m}(\tau)$. Thus the probability that an atom in eigenwave $n$ has aquired transverse momentum $m$ ink 0 is

$$
P_{m}=j_{m}^{2}\left(\Omega_{n} t\right)
$$

This result was obtained previously in II for the case of a two-level atom. The initial time development of $P_{m}$ is illustrated in Figure 2 . The distribution of probability over m spreads approximately linearly with time, and $P_{m}(t)$ (and hence $A_{m}$ ) are negligibly small except for $-\Omega_{n} t \leqslant m \lesssim \Omega_{n:} t$. Therefore, the first term on the right in (41) is indeed negligible so long as $\alpha\left(\Omega_{n} t\right)^{2} \ll 1$. The Bessel function expression for $P_{m}(t)$ constitutes a complete solution to the diffraction problem when the total interaction time $t_{L}=L / v_{z}$ satisfies the abo' inequality for all $\mathrm{n}$. The probability that the atom is deflected through angle $\theta_{m}=m k_{0} / k$ in the $x-z$ plane is the sum of the probabilities $P_{m}\left(t_{L}\right)$, Equation (43), for each eigenwave weighted by the probabilities $w_{n}$ that the eigenwaves are occupied,

$$
P\left(\theta_{m}\right)=\sum_{n=1}^{N} W_{n} J_{m}^{2}\left(\Omega_{n} t_{L}\right) .
$$


However, when the probability spreads to values of $m$ such that $\alpha m^{2} \sim 1$, the first term in (41) can no longer be neglected, and the Bessel function solution ceases to be valid.

It is known from general arguments based on the Raman-Nath equation, and from numerical solutions of the Raman-Nath equation, that the effect of the term $a m^{2} A_{m}$ in Equation (41) is to prevent spreading of probability to values of m much larger thar $\alpha^{-1 / 2}$. That is, this term limits the number of orders observed in the diffraction pattern. In the present context, this effect has a simple physical interpretation. Recall that in a standing wave of amplitude $E(x)=2 E_{0} \cos k_{0} x$ an atom in the $n$th eigenwave has potential energy $V(x)=-\frac{1}{2} \mu_{n} E(x)=-\mu_{n} E_{0} \cos k_{0} x$. The maximum potential difference that the atom can fall through in this potential is $\Delta V=2 \mu_{n} E_{0}$, and hence, the maximum transverse momentum that the field can deliver to the atom is $P_{X}=(2 M \Delta V)^{1 / 2}=2\left(M \mu_{n} E_{0}\right)^{1 / 2}$. Expressed in terms of $m$, this relation becomes $m \hbar k_{0}=2\left(M \mu_{n} E_{0}\right)^{1 / 2}$ or $\mathbf{m}=2^{1 / 2} \alpha^{-1 / 2}$. Therefore, the probability for diffraction into orders $m>2^{1 / 2} \alpha^{-1 / 2}$ is small because this is a classically disallowed region of momentum space. The very small value of $\alpha$ encountered in a strong standing light wave, $\alpha \sim 10^{-6}$ say, implies that the atom will be deflected into very high orders of the diffraction pattern, $m \sim 10^{3}$. The maximum deflection obtainable is $\theta \approx 2\left(M \mu_{n} E\right)^{l / 2} /$ thk where $\mu_{n}$ is the maximum eigenvalue for which the associated probability $W_{n}$ is nonnegligible. 
An explicit calculation of the distribution of atomc deflections $\theta_{m}$ for long interaction time $\left(t_{L} \geq \Omega_{n}^{-1} \alpha^{-1 / 2}\right)$ requires numerical evaluation of the exact solution, Equations (37), (32), and (33), or numerical solution of the Raman-Nath equation, Equation (41). The reader interested in persuing such calculations is referred to the extensive literature on techniques for numerical solution of the Raman-Nath equation ${ }^{32}$ which has developed along with the theory of diffraction of light by ultrasound. 
Chapter 5

ATOMIC MOTION IN RESONANT RADIATION:

AN APPLICATION OF EHRENFEST'S THEOREM 
Atomic Motion in Resonant Radiation:

An Application of Ehrenfest's Theorem

\author{
R. J. Cook \\ University of California \\ Lawrence Livermore Laboratory \\ P. 0. Box 808 \\ Livermore, California 94550
}

\title{
Abstract
}

A new theory of atomic motion in a resonant or near resonant electromagnetic wave, based on Ehrenfest's theorem and the optical Bloch equations, is presented. The theory provides a simple unified treatment of the radiation force including effects of spontaneous emission and induced dipole interactions. Analytical results are presented for a plane running wave, a general standing wave, a collimated Gaussian beam, and a combination of standing and running waves. 


\section{INTRODUCTION}

The recent revival of interest in the theory of atomic motion in an electromagnetic wave ${ }^{7,19,22,33-35}$ and the increasing number of proposals for the practical application of the theory ${ }^{16-20}$ suggest that this subject has a bright future in both pure and applied physics.

Existing theories of atomic motion in resonant radiation tend to fall into one of two catagories. There are elementary theories, based largely on intuition and on primative concepts such as cross-section and polarizability, that yield simple formulas for the radiation force under various circumstances, and provide clear physical pictures of the processes involved. These theories tend to be fragmented, with different arguments being used in the derivation of different aspects of the radiation force. For example, the radiation force associated with spontaneous emission and the radiation forcessociated with interaction of the induced atomic dipole moment with the amplitude gradient of the applied field are treated separately, and yet another argument is used in discussions of cooling of an atomic vapor by a standing wave tuned below resonance. Such fragmented arguments leave one with the uneasy feeling that perhaps some component of the total radiation force has been neglected, or that an interaction between the different effects might alter the results. On the other hand, there are theories that approach the atom-field interaction from first principles, with both the internal and translational motions of the atom treated quantum-mechanically, and often including interaction with 
the quantized electromagnetic field to take proper account of spontaneous emission. These theories tend to be rather cumbersome, and often numerical calculations must be carried out to obtain useful results.

The purpose of this paper is to present a new approach to the theory of atomic motion in a resonant or near resonant electromagnetic wave that may be classified approximately midway between the above two catagories. The theory, based on Ehrenfest's theorem and the optical Boch equation;, gives a unified treatment of the radiation force including effects of spontaneous emission and the induced dipole interaction, and, at the same time, retains much of the simplicity of previous elementary theories.

In the following section the theory is developed. In Sec. III explicit formulas are derived for the radiation force in a plane running wave, a standing wave, a collimated Gaussian beam, and a combination of standing and running waves. The paper concludes in Sec. IV with a summary of results and some comments on limitations of the theory.

\section{BASIC THEORY}

The Hamiltonian for an atom in a classically prescribed electromagnetic wave, in the electric dipole approximation, is

$$
H=P^{2} / 2 M+H_{0}-\vec{H} \cdot \vec{E}(\vec{R}, t),
$$

where $P^{2} / 2 M$ is the kinetic energy associated with the center-of-mass momentum $\vec{P}, H_{0}$ is the Hamiltonian for the internal motion of the unperturbed atom, $\vec{\mu}$ 
is the electric dipole moment operator, and $\vec{E}(\vec{R}, t)$ is the electric field evaluated at the center-of-mass position $\vec{R}$. In the Heisenberg representation, the operators $\vec{R}$ and $\vec{P}$ satisfy equations of motion

$$
\dot{\vec{R}}=(i K)^{-1}[\vec{R}, H]=\nabla_{P} H=\vec{P} / M
$$

and

$$
\vec{P}=(i h)^{-1}[\vec{P}, H]=-\nabla_{R} H=\nabla(\vec{\mu} \cdot \vec{E})
$$

respectively. Upon combining the expectation values of Equations (2) and (3), and setting $\vec{r}=\langle\vec{R}\rangle$, we obtain Ehrenfest's theorem

$$
\overrightarrow{\mathbf{F}}=\ddot{M r}=\langle\nabla(\vec{\mu} \cdot \vec{E})\rangle \text {. }
$$

To simplify the following calculation, we consider atomic motion in an electric field of the form $\vec{E}(\vec{x}, t)=\hat{\varepsilon} E(\vec{x}, t)$, with polarization vector $\hat{\varepsilon}$ independent of $\vec{x}$ and $t$. In this case, Equation (2) becomes $\vec{F}=\langle\vec{\mu} \cdot \hat{\varepsilon} \nabla E\rangle$, and if $\nabla E$ is nearly uniform across the atomic wave packet,

$$
\vec{F}=\langle\vec{\mu} \cdot \hat{\varepsilon}\rangle \nabla E(\vec{r}, t) .
$$

To the same approximation, the internal motion of the atom is driven by the electric vector $\vec{E}(t)=\hat{\varepsilon} E(\vec{r}(t), t)$ at the position of the moving atom, and the Hamiltonian for the internal motion is $H^{\prime}=H_{0}-\vec{\mu} \cdot \vec{E}(t)$.

Now consider the motion of a two-level atom, with energy levels $E_{7}$ and $E_{2}$, in an arbitrary monochromatic field

$$
\begin{gathered}
E(\vec{x}, t)=\frac{1}{2} E(\vec{x}) \exp \{i[\theta(\vec{x})+\omega t]\} \\
+ \text { c. c. }
\end{gathered}
$$


Le: $C_{1}$ and $C_{2}$ be the amplitudes that the atom is in levels $E_{1}$ and $E_{2}$ respectively. Then the Schrödinger equation for the internal motion of the atom is

$$
\begin{aligned}
& i \mu \dot{C}_{1}=E_{1} C_{1}-\mu E(t) C_{2} \\
& i k \dot{C}_{2}=E_{2} C_{2}-\mu E(t) C_{1}
\end{aligned}
$$

where $\mu=\langle 1|\vec{\mu} \cdot \hat{\varepsilon}| 2\rangle$ is the transition dipole moment (here taken to be rea1), and $E(t)=E(\vec{r}(t), t)$. Upon substituting the relations

$$
\begin{aligned}
& C_{1}=D_{1} \exp \left\{-i E_{1} t / h+\frac{1}{2} i[\Delta t+\theta(t)]\right\} \\
& C_{2}=D_{2} \exp \left\{-i E_{2} t / h-\frac{1}{2} i[\Delta t+\theta(t)]\right\}
\end{aligned}
$$

into Equations (7), with $\Delta=\omega-\omega_{0}\left(\omega_{0}=\left(E_{2}-E_{1}\right) / h\right)$ and $\theta(t)=\theta(\vec{r}(t))$, Equations (7) become

$$
\begin{aligned}
& i \mu \dot{D}_{1}=\frac{1}{2} h(L+\dot{\theta}) D_{1}-\mu E D_{2} \exp \{-i(\theta+\omega t)\} \\
& i H D_{2}=-\frac{1}{2} n(\Delta+\dot{\theta}) D_{2}-\mu E D_{1} \exp \{i(\theta+\omega t)\}
\end{aligned}
$$

Then inserting ( 6 ) into Equations (9) and neglecting inessential terms that oscillate at twice the optical frequency (rotating wave approximation) ${ }^{12}$, Equations (9) reduce to

$$
\begin{aligned}
& i \mu \delta_{1}=\frac{1}{2} H[\Delta+\dot{\theta}(t)] D_{1}-\frac{1}{2} \mu E(t) D_{2} \\
& i H \dot{D}_{2}=-\frac{1}{2} H[\Delta+\dot{\theta}(t)] D_{2}-\frac{1}{2} \mu E(t) D_{1}
\end{aligned}
$$


Note that $E(t)$ and $\dot{\theta}(t)$ are determined by the atomic position and velocity through the relations $E=E(\vec{r})$ and $\dot{\theta}=\nabla \theta(\vec{r}) \cdot \dot{\vec{r}}$.

According to Equations (8), the density matrix for the internal motion, $\rho_{\mathrm{nm}}=\mathrm{C}_{\mathrm{n}} \mathrm{C}_{\mathrm{m}}{ }^{*}$, can be written as

$$
\begin{aligned}
& \rho_{11}=\sigma_{11} \\
& \rho_{22}=\sigma_{22} \\
& \rho_{12}=\sigma_{12} \exp \{i(\theta+\omega t)\} \\
& \rho_{21}=\sigma_{21} \exp \{-i(\theta+\omega t)\}
\end{aligned}
$$

where $\sigma_{n m}=D_{n} D_{m}^{*}$, and it follows from Equations (10) that $\sigma_{n m}$ satisfy equations of motion

$$
\begin{aligned}
& \dot{\sigma}_{11}=-\frac{1}{2} i_{\Omega}\left(\sigma_{12}-\sigma_{21}\right) \\
& \dot{\sigma}_{22}=\frac{1}{2} i \Omega\left(\sigma_{12}-\sigma_{21}\right) \\
& \dot{\sigma}_{12}=-i(\Delta+\dot{\theta}) \sigma_{12}+\frac{1}{2} i \Omega\left(\sigma_{22}-\sigma_{11}\right)
\end{aligned}
$$

where $\Omega(t)=\mu E(t) / h . \quad \Omega=\mu E / h$ is the on-resonance Rabi flopping frequency for a two-level atom in a field of amplitude E.

The expectation value appearing in Equation (5) is written in terms of the density matrix, or in terms of $\sigma_{n m}$, as

$$
\begin{aligned}
\langle\vec{\mu} \cdot \hat{\varepsilon}> & =\mu\left(\rho_{12}+\rho_{21}\right) \\
=\mu\left[\sigma_{12}\right. & \exp \{i(\theta+\omega t)\} \\
& \left.+\sigma_{21} \exp [-i(\theta+\omega t)\}\right] .
\end{aligned}
$$


Here $\sigma_{n m}$ are slowly varying functions of time compared to the optical factor $\exp \{i \omega t\}$. The equation of motion for the atom is obtained by substituting (6) and (13) into Equation (5) and again discarding inessential terms that oscillate at twice the optical frequency. The result is

$$
\begin{aligned}
\overrightarrow{\mathrm{F}} & =\ddot{M r}=\frac{1}{2} \mu V E\left(\sigma_{12}+\sigma_{21}\right) \\
& -\frac{1}{2} i_{\mu E \nabla \theta}\left(\sigma_{12}-\sigma_{21}\right) .
\end{aligned}
$$

Effects of spontaneous emission are introduced into the theory by adding relaxation terms to Equations (12),

$$
\begin{aligned}
& \dot{\sigma}_{11}=-\frac{1}{2} i \Omega\left(\sigma_{12}-\sigma_{21}\right)+A \sigma_{22}, \\
& \dot{\sigma}_{22}=\frac{1}{2} i \Omega\left(\sigma_{12}-\sigma_{21}\right)-A \sigma_{22}, \\
& \dot{\sigma}_{12}=-i(\Delta+\dot{\theta})_{12}+\frac{1}{2} i \Omega\left(\sigma_{22}-\sigma_{11}\right)-\frac{1}{2} A \sigma_{12},
\end{aligned}
$$

where $A=4 \omega_{0}^{3}|<1| \vec{\mu}|2>|^{2} / 3 / c^{3}$ is the spontaneous emission rate (Einstein A coefficient). The relaxation terms nay be derived from a first-principles calculation ${ }^{36}$ or simply written down on the basis of simple phenomenological arguments.

Equations (14) and (15) can be rewritten in terms of three real variables

$$
\begin{aligned}
& u=\left(\sigma_{12}+\sigma_{21}\right) \\
& v=-i\left(\sigma_{12}-\sigma_{21}\right) \\
& \mathbf{W}=\left(\sigma_{22}-\sigma_{11}\right)
\end{aligned}
$$


as

$$
\vec{F}=\ddot{M r}=\frac{1}{2} h\left[U \nabla \Omega+V_{\Omega} \theta \theta\right]
$$

and

$$
\begin{aligned}
& \dot{U}=(\Delta+\dot{\theta}) V \quad-\frac{1}{2} A U \\
& \dot{V}=-(\Delta+\dot{\theta}) U+\Omega W-\frac{1}{2} A V \\
& \dot{W}=\quad-s V-A(W+1)
\end{aligned}
$$

respectively, where $\sigma_{11}+\sigma_{22}=1$ was used in the derivation of (18). Equations (18) are the optical Bloch equations in the rotating wave approximation .

Equations (17) and (18) determine the motion of a two-Tevel atom in a monochromatic field with arbitrary amplitude $E(\vec{x})$ and phase $\theta(\vec{x})$. It is clear from these equations that, in general, the radiation force is not a simple function of the atoms position and velocity, but rather depends on the history of the motion through the Bloch equations. There are, however, certain cases in which the radiation force reduces to a function of atomic position and velocity to an excellent approximation. Some of these cases are discussed in the following section.

\section{EXAMPLES}

A. Steady-state approximation

Consider first the case in which the electric field amplitude $E(t)=E(\vec{r}(t))$ and phase derivative $\dot{\theta}(t)=\nabla_{\theta}(\vec{r}(t)) \cdot \dot{\vec{r}}(t)$ vary by only a small fraction during a natural lifetime $\tau_{N}=1 / A$, i.e., the case in 
which the atom moves sufficientiy slowly so that at each instant $U, V$, and $W$ assume the steady-state values obtained from Equatirns (18) by setting $\dot{U}=\dot{V}=\dot{W}=0$. The steady-state solution of Equations (18) gives

$$
\begin{aligned}
& U=-\frac{4 \Omega(\Delta+\dot{\theta})}{4(\Delta+\dot{\theta})^{2}+A^{2}+2 \Omega^{2}}, \\
& V=-\frac{2 A \Omega}{4(\Delta+\dot{\theta})^{2}+A^{2}+2 \Omega^{2}},
\end{aligned}
$$

and hence, the radiation force, Equation (17), becomes

$$
\vec{F}=-\frac{h A \Omega^{2} \nabla \theta+h(\Delta+\dot{\theta}) \nabla \Omega^{2}}{4(\Delta+\dot{\theta})^{2}+A^{2}+2 \Omega^{2}}
$$

The meaning of Equation (19) will be illustrated by applying it to a few: simple problems.

For a plane running wave, $E(\vec{x}, t)=E_{0} \cos (\vec{k} \cdot \vec{x}-\omega t)$, we ha se $\Omega=\mu E_{0} / h=$ constant, $\theta(\vec{x})=-\vec{k} \cdot \vec{x}$, and $\dot{\theta}=-\vec{k} \cdot \dot{\vec{r}}$. The radia ion force, Equation (19), reduces to

$$
\vec{F}=\frac{A \Omega^{2} h \vec{k}}{4\left(\Delta-\vec{k} \cdot \frac{\dot{q}}{r}\right)^{2}+A^{2}+2 \Omega^{2}}
$$

This is the radiation force associated with spontaneous en ission or with scattering of radiation by the atom. The force is a Lorentzian function of w centered at $\omega_{0}+\vec{k} \cdot \vec{r}$ (atomic frequency flus Doppler shift) with fult width at haif maximum $\left(A^{2}+2 \Omega^{2}\right)^{1 / 2}$ corresporiding to natural and power broadening of the atomic responce. In a strong field $(\Omega+\infty)$ the force saturates to the value $\vec{F}=\frac{1}{2} A k \vec{k}$. Equation $(20)$ is consistent with Ashkins theory of resonance-radiation pressure. 19 
In a general standing wave, $E(\vec{x}, t)=E(\vec{x}) \cos \omega t$, we have $\Omega(\vec{x})=\mu E(\vec{x}) / k$ and $\theta=0$. Here the amplitude $E(\vec{x})$ is a solution of the time-independent wave equation $\nabla^{2} E+(\omega / c)^{2} E=0$. The radiation force, Equation (19), is now

$$
\vec{F}=-\frac{h \Delta \nabla \Omega^{2}}{4 \Delta^{2}+A^{2}+2 \Omega^{2}}
$$

This force is a result of the interaction between the induced atomic dipole moment and the amplitude gradient of the standing wave. It may be written in the form $\vec{F}=\frac{1}{2} \alpha \nabla E^{2}$, where $\alpha=-2 \Delta \mu^{2} / h\left[4 \Delta^{2}+A^{2}+2(\mu E . h)^{2}\right]$ is the atomic polarizahility. The dipole force is derivable from a potential

$$
\begin{aligned}
& \vec{F}=-\nabla U, \\
& U=\frac{1}{2} \not \Delta \Delta \ln \left(4 \Delta^{2}+A^{2}+2 \Omega^{2}\right) .
\end{aligned}
$$

When the field is tuned above resonance $(\Delta>0)$ the dipole force is in the direction of decreasing field strength, and the atom tends to be expelled by the field. When the field is tuned below resonarice $(\Delta<0)$ the dipole force is in the direction of increasing field strength, and the atom tends to he trapped by the radiation. On resonance $(\Delta=0)$ the dipole force vanishes. These results for a general standing wave are again consistent with the theory of Ashkin."

Equation (19) may also be applied to problems jnvolving a combination of standing and running waves. In this case, a new velocity-dependent term appears which has not been considered in previous treatments of radiation force. For example, if the applied field 


$$
\begin{aligned}
E(x, t) & =E_{S} \cos k x \cos \omega t \\
& +E_{T} \cos (k x-\omega t)
\end{aligned}
$$

is written in the form of Equation (6), and the resulting amplitude $E(x)$ and phase $\theta(x)$ are inserted into Equation (19), the radiation force secomes

$$
\begin{aligned}
F_{x}= & A \Omega_{1} \Omega_{2} \not k k / D-H \Delta\left[\Omega^{2}\right] \cdot / D \\
& +h \Omega_{1} \Omega_{2} k \dot{x}\left[\Omega^{2}\right] / /\left(\Omega^{2} D\right)
\end{aligned}
$$

where $\Omega_{1}=\mu E_{T} / h, \Omega_{2}=\mu\left(E_{T}+E_{S}\right) / h$, and

$$
\begin{aligned}
& \Omega^{2}=\Omega_{1}^{2} \operatorname{sir}^{2} k x+\Omega_{2}^{2} \cos ^{2} k x, \\
& D=4(\Delta+\dot{\theta})^{2}+A^{2}+2 \Omega^{2}, \\
& \dot{\theta}=-\Omega_{1} \Omega_{2} k \dot{x} / \Omega^{2}
\end{aligned}
$$

The first two terms in (24) will be recognized as generalizations of the running and standing viave forces considered above, while the third term is new and occurs only when standing and running waves are simultaneously present. When the atomic velocity is zero, the new term vanishes, and the dipole force in (24) is derivable from the potential (22) with $\Omega^{2}(x)$ taken from Equation (25). The depth of modulation of this periodic potential is

$$
\begin{gathered}
\delta U=U_{\max }-U_{\min } \\
=\frac{1}{2} h \Delta L n\left[\frac{4 \Delta^{2}+A^{2}+2\left(\Omega_{s}+\Omega_{\eta}\right)^{2}}{4 \Delta^{2}+A^{2}+2 \Omega_{1}^{2}}\right]
\end{gathered}
$$


where $\Omega_{S}={ }_{\mu} E_{S} / H$. As the strength of the running wave $\Omega_{1}$ increases from zero, the trapping energy $\delta U$ of the standing wave first increases to a maximum and then decrease to zero as $h_{\Delta}\left(\Omega_{s} / \Omega_{1}\right)=h_{\Delta}\left(E_{s} / E_{1}\right)$. This result disagrees with the theory of Ashkin ${ }^{19}$ which predicts a monotonic decrease of the trapping energy with increasing $E_{1}$, and a limiting value proportional to $\left(E_{s} / E_{1}\right)^{2}$ as $E_{1} \rightarrow \infty$.

Another case of current experimental interest is that of atomic motion in a Gaussian laser beam. Consider a collimated Gaussian beam of spot size $w_{0}$ propagating in the $z$ direction

$$
E(\vec{x}, t)=E_{0} \exp \left\{-\left(x^{2}+y^{2}\right) / w_{0}^{2}\right\} \cos \{k z-\omega t\} .
$$

Here $\Omega(x, y)=\left(\mu E_{0} / h\right) \exp \left\{-\left(x^{2}+y^{2}\right) / w_{0}^{2}\right\}$ and $\theta(z)=-k z$. The radiation force, Equation (19), consists of a longitudinal radiation pressure

$$
F_{Z}=\frac{A \Omega^{2} \eta k}{4(\Delta-k \dot{z})^{2}+A^{2}+2 c^{2}}
$$

and a transverse dipole force

$$
\vec{F}_{T}=-\frac{h(\Delta-k \dot{z}) \nabla \Omega^{2}}{4(\Delta-k \dot{z})^{2}+A^{2}+2 \Omega^{2}}
$$

Untike the dipole force in a standing wave, which is independent of velocity (in the present approximation), the transverse dipole force depends on the atomic velocity through the Doppler shift $k \dot{z}$. The dipole force is directed toward the beam axis when $k \dot{z}>\Delta$ and away from the axis when $k \dot{z}<\Delta$. This result leads to the interesting prediction that in a resonant Gaussian 
beam $(\Delta=0)$ a co-propagating atomic beam $(\dot{z}>0)$ is focussed and trapped by the field, while a counter-propagating atomic beam $(\dot{z}<0)$ is defocussed and expelled by the resonant radiation. Focussing and defocussing of a beam of sodium atoms by the transverse dipole force in a co-propagating Gaussian laser beam has recently been observed in the experiment of Bjorkholm et at?

B. Dissipative force in a standing wave

When an atom moves with typical thermal velocity $v$ across the fringes of a simple standing wave $E(x)=E_{0} \cos k x$ of visible light, the approximation of slowly varying field amplitude that lead to Equation (19) is no longer valid (the amplitude at the moving atom varies as $E(t)=E_{0} \cos k v t$ and $k v$ generally exceeds the spontaneous emission rate A). Thus the above standingwave results are valid only in the limit $k v<<A$, and the case $k v \geqslant A$ requires a different approach. In this subsection we calculate the time-average radiation force for arbitrary atomic velocity in a weak standing wave. This probiem is of considerable interest in connection with recent proposals for cooling an atomic vapor by a standing wave tuned below resonance. 18,19

In a simple standing wave $\Omega(x)=\Omega_{0} \cos k x\left(\Omega_{0}=\mu E_{0} / k\right), \theta=0$, and the force acting on the atom, Equation (17), is $F_{x}=-\frac{1}{2} J_{\Omega_{0}}$ hik $\sin k x$. Let $x=v t$. Then the time-average radiation force is

$$
F_{X}=-\frac{1}{2} \Omega_{0} \forall k<U(\tau) \operatorname{sinkvt>}{ }_{a v},
$$


and the equations describing the internal notion of the atom, Equations (18), are

$$
\begin{aligned}
& \dot{U}=-\frac{1}{2} A U+\Delta V \\
& \dot{V}=-\frac{1}{2} A V-\Delta U+\Omega_{0} \cos (k v t) W \\
& \left.\dot{W}=-\Omega_{0} \cos (k v t) V-A(!)+1\right)
\end{aligned}
$$

If the field is weak $\left(\Omega_{0}<<\right)$, the degree of atomic excitation (or inversion) $W$ remains near the ground state value $(W \approx-1)$. For $H=-1$ the first two of Equations (31) can be solved exactly. The persistent solution is

$$
\begin{aligned}
& U=\alpha \cos k v t+\beta \sin k v t \\
& V=\gamma \cos k v t+v \sin k v t
\end{aligned}
$$

where

$$
\begin{aligned}
& \alpha=-\Delta \Omega_{0}\left[(A / 2)^{2}+\Delta^{2}-(k v)^{2}\right] / D \\
& B=-\Delta \Omega_{0} A k v / D \\
& \gamma=-\frac{1}{2} A \Omega_{0}\left[(A / 2)^{2}+\Delta^{2}+(k v)^{2}\right] / D \\
& \nu=-\Omega_{0} k v\left[(A / 2)^{2}-\Delta^{2}+(k v)^{2}\right] / D
\end{aligned}
$$

and

$$
D=\left[\Delta^{2}-(k v)^{2}\right]^{2}+(A / 2)^{2}\left[(A / 2)^{2}+2 \Delta^{2}+2(k v)^{2}\right] .
$$


insertion of (32) into (30) yาelds $F_{X}=-\frac{i}{4} \Omega_{0} k k \beta$, and taking $\beta$ from (34) we obtain

$$
\bar{F}_{X}=\frac{v \wedge A \Omega_{o}^{2} h k^{2}}{4\left[\Delta^{2}-(k v)^{2}\right]^{2}+A^{2}\left[(A / 2)^{2}+2 \Delta^{2}+2(k v)^{2}\right]}
$$

This equation states that the time-average radiation force in a weak standing wave is a positive quantity trmes vo. Thus a standing wave tuned below resonance $(\Delta<0)$ damps the atomic velocity, while a standing wave tuned above resonance $(\Delta>0)$ amplifies the atomic velocity.

A reliable calculation of the radiation force in a strong standing wave requires solution of the full set of equations (31). This problenl is mora difficult than the simple examples considered here and will not be discussed in the present paper.

\section{CONCLUSION}

The purpose of this paper has been to present a simple unified theory of atomic motion in resonant radiation, and to demonstrate the utility of the theory by applying it to a number of problems of current interest. The spontaneous force in a plane running wave, the dipole force in a general standing wave, and the transverse dipole force in a collimated Gaussian beam were obtained by almost trivial applications of the theory in the steady-state approximation. New results were presented for the radiation force in a combination of standing and running waves, and a formula for tine dissipative force in a weak standing wave was derived. 
It should be emphasized that a theory based on Ehrenfest's theorem describes the motion of the centroid of the center-of-mass probability density. It says nothing about the spread of the atomic wave packet about the centroid. Because of this limitation, results of the present theory are, in some cases, misleading. For example, the present theory suggests that the radiation force acting on a slowly moving atom in a standing wave vanishes as $\Delta \rightarrow 0$, while a more detailed theory ${ }^{24}$ shows that, for $\Delta=0$, the atomic trajectory is split by the resonant radiation, in much the same way as a narrow atonic beam is split in the Stern-Gerlach experiment, but the splitting is symmetric so the centroid is not accelerated. This lack of detail in the present theory is the price paid for simplicity. On the other hand, in almost all problems of practical interest, the deBroglie wavelength of the atom is many orders of magnitude smaller than the optical wavelength (the minimum scale size of the applied field), and therefore it is expected that the simple picture of a point atom moving along a classical trajectory is an excellent approximation for a wide class of problems.

The present theory is clearly applicable to more elaborate problems than considered here. We believe that our basic working equations, Eqs. (17) and (18), will provide a convenient and fruitful framework in which to study such problems. 
Chapter 6

ATOMIC MOTION IN RESONANT FLUCTUATING LASER RADIATION 


\title{
ATOMIC MOTION IN RESONANT FLUCTUATING
}

LASER RADIATION

$$
\text { R.J. Cook }
$$

\author{
University of California \\ Lawrence Livermore Laboratory \\ P.0. Box 5508 \\ Livermore, California 94550
}

\begin{abstract}
The effect of field fluctuations on the motion of a two-level atom in resonant laser radiation is investigated theoretically. The internal motion of the atom is treated quantum-mechanically (optical Bloch equations), the translational motion, which is coupled to the internal motion, is treated classically (Ehrenfest's theorem), and the fluctuating radiation is treated as a classical electromagnetic wave with stochastic phase (phase-diffusion model). An expression for the radiation force and equations of motion for the ensemble-averaged Bloch vector are derived and used to calcuiate: (1) the radiation force in a plane running wave, (2) the steady-state radiation force in a general standing wave, and (3) the dissipative force in a plane standing wave in the limit of strong phase fluctuations. In case (3) it is found that the atomic velocity is damped when the radiation is tuned above resonance, which is opposite to the case of a weak coherent standing wave in which damping occures
\end{abstract}


for radiation tuned below resonance. It is shown that fluctuations have a substantial effect on the radiation force only when the spectral width of the radiation approaches or exceeds the spontaneous emission rate $A$. 


\section{INTRODUCTION}

The theory of atomic motion in resonant radiation has attracted considerable interest in recent years not only because of a basic desire to understand this aspect of the interaction of radiation with matter but also because of the possibility of applying the theory to problems of laser isotope separation $4,10,28$, atomic trapping and cooling ${ }^{17-19,35,37}$, neutral-atom acceleration 22,25 , atomic-beam-deflection spectroscopy ${ }^{2,5,11}$, and so on.

Although all real sources of radiation undergo fluctuations and have finite bandwidths, existing theories of atomic motion in resonant radiation have largely ignored the fluctuations, and have treated laser radiation as if it were perfectly monochromatic. Questions naturally arise, therefore, as to what effect fluctuations might have on the atomic motion, or to what extent the radiation force acting on the atom is influenced by the finite coherence time of the applied field. The purpose of this paper is to take a first step toward answering these questions.

In a recent paper 38 it was shown that the motion of a two-level atom of mass $M$, resonant frequency $\omega_{0}$, transition dipole moment $\mu$, and spontaneous emission rate $A$, in a general monochromatic field

$$
E(\vec{x}, t)=E(\vec{x}) \cos \{u t+\theta(\vec{x})\} \text {, }
$$

is determined by the coupled set of equations

$$
M \ddot{\vec{r}}=(1 / 2) \hbar[u \nabla \Omega+v \Omega \nabla \theta]
$$




$$
\begin{array}{ll}
\dot{u}=(\Delta+\dot{\theta}) v & -(1 / 2) A u \\
\dot{v}=-(\Delta+\dot{\theta}) u & +\Omega_{w-}(1 / 2) A v \\
\dot{w}= & -\Omega_{v-A}(w+1)
\end{array}
$$

where $\Omega(\vec{r})=U E(\vec{r}) / \hbar$ is the on-resonance Rabi flopping frequency, $(u, v, w)$ is the Bloch vector in the rotating wave approximation, an: $\Delta=\omega-\omega_{0}$ is the detuning frequency. In Eq. (2) $\nabla \Omega, \Omega$, and $\nabla \theta$ are functions of atomic position $\vec{r}$, and $u$ and $v$ are functions of time determined by Eqs. (3), while in Eqs. (3) $\dot{\theta}(t)=\nabla \theta(\vec{r}) \cdot \dot{\vec{r}}$ and $\Omega(t)=\Omega(\vec{r})$ are regarded as functions of time determined by the atomic trajectory $\vec{r}(t)$. Fquation (2) was derived from Ehrenfest's theorem, and Eqs. (3) are the usual optical Bloch equations, here driven by the electric field at the moving atom.

In the present paper we shall extend Eqs. (2) and (3) to the case in which the electric field contains an additional stochastic phase $\phi(t)$,

$$
E(\vec{x}, t)=E(\vec{x}) \cos \{\omega t+\theta(\vec{x})+\phi(t)\},
$$

and the phase derivative $\dot{\phi}(t)$ is $\delta$-correlated in time, the so-ca.led phase-diffusion model of laser fluctuations ${ }^{39}$. The phase-diffusion mode] is motivated by the fact that radiation emanating from a single-mode laser operated far above threshold is well represented by a classical electromagnetic wave with fixed amplitude and randomly drifting phase ${ }^{39,40}$. It should be emphasized that by taking the amplitude $E(\vec{x})$ to be fixed and 
the stochastic phase $\phi(t)$ to be independent of position, we are restricting the theory to fluctuating fields of a rather special form. The field $E(\vec{x}, t)$ in the interaction region will be of this form when the interaction region is small compared to the coherence length of the source laser and when all optical path lengths from the source to the interaction region, through the optics that generate the desired field $E(\vec{x}, t)$, differ by much less than a coherence length. For exanple, if a laser beam is $f$. ' " back on itself by a plane mirror to form a standing wave, the field will be of the form in Eq. (4) in the region adjacent to the mirror where the "visibility" of the standing wave interference pattern is near unity.

In the following section the statistics of a field with fixed amplitude and $\delta$-correlated phase derivative is briefly reviewed. In section III an expression for the mean radiation force is written down, and the equations of motion for the ensemble-averaged Bloch vector are derived. In section IV the theory is applied to a plane running wave and a general standing wave in the steady-state approximation, and finally, in section $V$, the dissipative radiation force in a plane standing wave is calculated in the limit of broad-spectrum radiation. 


\section{FIELD STATISTICS}

The electric field, Eq. (4), is the real part of

$$
\tilde{E}(\vec{x}, t)=E(\vec{x}) \exp \{-i[\omega t+\theta(\vec{x})+\phi(t)]\} .
$$

We assume that the phase derivative $\dot{\phi}(t)$ has mean value zero and is $f$-correlated in time

$$
\begin{aligned}
& \langle\dot{\phi}(t)\rangle=0, \\
& \left\langle\dot{\phi}(t) \quad \dot{\phi}\left(t^{\prime}\right)\right\rangle=2 \Gamma \delta\left(t^{\prime}-t\right) .
\end{aligned}
$$

It follows from these relations that the phase change

$$
\phi(t)-\phi(0)=\int_{0}^{t} \dot{\phi}(s) d s
$$

has mean zero and variance

$$
\begin{aligned}
\left\langle[\phi(t)-\phi(0)]^{2}\right\rangle & =\int_{0}^{t} d s \int_{0}^{t} d s^{\prime}\left\langle\dot{\phi}(s) \dot{\phi}\left(s^{\prime}\right)\right\rangle \\
& =2 \Gamma t .
\end{aligned}
$$

The stochastic phase $\phi(t)$ is a constant $\phi(0)$ plus the sum of a large number of statistically independent impulses $\dot{\phi}(s) d s$. Therefore, by the central limit theorem $41, \phi(t)$ is Gaussian distributed. It has been emphasized by several authars that the statistics of the diffusing phase $\phi(t)$ is formally analogous to the statistics of Brownian motion in one dimension 42,43 . 
The temporal correlation function of the electric field $\tilde{E}$, at some prescribed point $\vec{x}$, is

$$
\left\langle\tilde{E}(t) \tilde{E^{\star}}\left(t^{\prime}\right)\right\rangle=E^{2} e^{i \omega\left(t^{\prime}-t\right)}\left\langle e^{i\left[\phi\left(t^{\prime}\right)-\phi(t)\right]}\right\rangle,
$$

and because $\phi(t)$ is a Gaussian stochastic process

$$
\left\langle\mathrm{e}^{i\left[\phi\left(t^{\prime}\right)-\phi(t)\right]}\right\rangle=\mathrm{e}^{-(1 / 2)\left\langle\left[\phi\left(\mathrm{t}^{\prime}\right)-\phi(t)\right]^{2}\right\rangle} .
$$

Using (8) and then (7), we find that

$$
\begin{aligned}
\left\langle\left[\phi\left(t^{\prime}\right)-\phi(t)\right]^{2}\right\rangle & =\int_{t}^{t^{\prime}} d s \int_{t}^{t^{\prime}} d s^{\prime}\left\langle\dot{\phi}(s) \dot{\phi}\left(s^{\prime}\right)\right\rangle \\
& =2 \Gamma\left|t^{\prime}-t\right| .
\end{aligned}
$$

Hence the correlation function $\left\langle\tilde{E}(t) \tilde{E}^{*}\left(t^{\prime}\right)\right\rangle=c\left(t^{\prime}-t\right)$ has the form

$$
C(\tau)=E^{2} e^{i \omega \tau-\Gamma|\tau|},
$$

and the spectrum of the radiation,

$$
\begin{aligned}
I\left(\omega^{\prime}\right) & =\int_{\infty}^{\infty} l(\tau) e^{-i \omega^{\prime} \tau} d \tau \\
& =\frac{2 \Gamma E^{2}}{\left(\omega^{\prime}-\omega\right)^{2}+\Gamma^{2}},
\end{aligned}
$$

is seen to be Lorentzian with spectral width $\Delta \omega^{\prime}=\Gamma$. 
II1. EQUATIONS OF MOTION

The only change of Eqs. (3) resulting from the addition of the stochastic phase $\phi(t)$ to the monochromatic field (1) is that $\dot{e}(t)$ is replaced by $\dot{\theta}(t)+\dot{\phi}(t)$. The Bloch equations become

$$
\begin{array}{lr}
\dot{u}=(\Delta+\dot{\theta}+\dot{\phi}) v & -(1 / 2) A u \\
\dot{v}=-(\Delta+\dot{\theta}+\dot{\phi}) u+\Omega w-(1 / 2) A v \\
\dot{w}= & -\Omega v-A(w+1)
\end{array}
$$

The equations of motion for the ensemble-averaged BToch vector $(\vec{u}, \vec{v}, \vec{w})$ may be derived from Eqs. (15) without approximations by using the theory of multiplicative stochastic processes when $\dot{\phi}(t)$ is $\delta$-correlated in time. For completeness we shall present here a simple approximate calculation leading to the same result, and refer the interested reader to the literature 44,45 for more rigorous treatments of this probler.

We define vectors

$$
X=\left[\begin{array}{l}
u \\
v \\
w
\end{array}\right], \quad c=\left[\begin{array}{c}
0 \\
0 \\
-A
\end{array}\right]
$$

and mati-ices

$$
\begin{aligned}
& M=\left[\begin{array}{ccc}
-A / 2 & \Delta+\dot{\theta} & 0 \\
-(\Delta+\dot{\theta}) & -A / 2 & \Omega \\
0 & -\Omega & -A
\end{array}\right], \\
& N=\left[\begin{array}{ccc}
0 & ? & 0 \\
-1 & 0 & 0 \\
0 & 0 & 0
\end{array}\right] .
\end{aligned}
$$




$$
\dot{x}=(M+\dot{\phi} N) x+c
$$

Next we integrate this equation over a time interval $t_{0}$ to $t$ sufficiently short that $M(t)$ may be regarded as constant over the interval,

$$
x(t)=x\left(t_{0}\right)+C\left(t-t_{0}\right)+M \int_{t_{0}}^{t} x(s) d s+N \int_{t_{0}}^{t} \dot{\phi}(s) x(s) \text { ds } .
$$

Equation (20) is solved by iteration. The zeroth order solution is $X(t)=X\left(t_{0}\right)$. Upon substituting this on the right in $(20)$ we obtain the first order solution

$$
x(t)=x\left(t_{0}\right)+C\left(t-t_{0}\right)+M X\left(t_{0}\right)\left(t-t_{0}\right)+N X\left(t_{0}\right) \int_{t_{0}}^{t} \dot{\phi}(s) d s .
$$

A second iteration yields the second order solution

$$
\begin{aligned}
x(t)-x\left(t_{0}\right)=\left[\left(t-t_{0}\right)+\frac{1}{2} M\left(t-t_{0}\right)^{2}+N \int_{t_{0}}^{t} d s \dot{\phi}(s)\left(s-t_{0}\right)\right] c \\
+M\left[\left(t-t_{0}\right)+\frac{1}{2} M\left(t-t_{0}\right)^{2}+N \int_{t_{0}}^{t} d s \int_{t_{0}}^{s} d s^{\prime} \dot{\phi}\left(s^{\prime}\right)\right] x\left(t_{0}\right) \\
+N\left[\int_{t_{0}}^{t} d s \dot{\phi}(s)+M \int_{t_{0}}^{t} d s \dot{\phi}(s)\left(s^{\prime}-t_{0}\right)\right. \\
\left.+N \int_{t_{0}}^{t} d s \int_{t_{0}}^{s} d s^{\prime} \dot{\phi}(s) \dot{\phi}\left(s^{\prime}\right)\right] x\left(t_{0}\right)
\end{aligned}
$$

We now take the ensemble average of (22) noting that $x\left(t_{0}\right)$ and $\dot{\phi}(t)$, for $t>t_{0}$, are statistically independent because $x\left(t_{0}\right)$ depends upon $\dot{\phi}(t)$ only for $t<t_{0}$ and $\dot{\phi}(t)$ is o-correlated in time. Equation (6) indicates that all terms linear in $\dot{\phi}$ vanish when averaged, and hence 
(22) yields

$$
\begin{aligned}
\frac{X(t)-\bar{x}\left(t_{0}\right)}{t-t_{0}}= & {\left[1+\frac{1}{2} M\left(t-t_{0}\right)\right]\left[M \bar{X}\left(t_{0}\right)+c\right] } \\
& +\frac{1}{t-t_{0}} \int_{t_{0}}^{t} d s \int_{t_{0}}^{s} d s^{\prime}<\dot{\phi}(s) \dot{\phi}\left(s^{\prime}\right)>N^{2} \bar{x}\left(t_{0}\right)
\end{aligned}
$$

Finally, with the help of Eq. (7), the double integral in (23) is readily evaluated, and passing to the limit $t \rightarrow t_{0}$ we obtain the equation of motion for the ensemble-averaged Bloch vector

$$
\overline{\bar{X}}=\left(M+\Gamma N^{2}\right) \bar{X}+C,
$$

or in component form

$$
\begin{aligned}
& \dot{\bar{u}}=(\Delta+\dot{\theta}) \bar{v} \quad-(1 / 2)(A+2 \Gamma) \bar{u} \\
& \dot{\bar{v}}=-(\Delta+\dot{\theta}) \bar{u}+\sin -(1 / 2)(A+2 \Gamma) \bar{v} \\
& \dot{\bar{w}}=\quad-\Omega \bar{v}-A(\bar{w}+1)
\end{aligned}
$$

The net effect of the stochastic phase on the rloch equations is to change the transverse relaxation rate $\left(R^{\prime}=1 / T_{2}\right)$ from $A / 2$ to $(A+2 \Gamma) / 2$. The longitudinal relaxation rate $\left(R_{1}=1 / T_{1}=A\right)$ is not affected by the fluctuating field.

The ensemble average of the radiation force, Eq. (2), is simply

$$
\vec{F}=\dot{M r}=\frac{1}{2} \hbar[\bar{u} \nabla \Omega+\bar{v} \Omega \nabla \theta]
$$

This equation together with Eqs. (25) determine the atomic trajectory 
in the fluctuating radiation.

\section{STEADY-STATE APPROXIMATION}

When the atomic velocity is small or when $\nabla \theta(\vec{x})$ and $\Omega(\vec{x})$ are slowly varying functions of position, the quantities $\dot{\theta}(t)=\nabla \theta(\vec{r}) \cdot \dot{\vec{r}}$ and $\Omega(t)=\Omega(\vec{r})$, that drive the Bloch equations, are slowly varying functions of time. In this case, the Bloch vector $(\bar{u}, \bar{v}, \bar{w})$ is, at each instant, very nearly equal to the "steady-state" Bloch vector obtained from Eqs. by setting $\dot{\bar{u}}=\dot{\bar{v}}=\dot{\bar{w}}=0$, namely

$$
\begin{aligned}
& \bar{u}=-4 \Omega(\Delta+\dot{\theta}) / D \\
& \bar{v}=-2(A+2 \Gamma) \Omega / D \\
& \bar{w}=-\left[4(\Delta+\dot{\theta})^{2}+(A+2 \Gamma)^{2}\right] / D
\end{aligned}
$$

where

$$
D=4(\Delta+\dot{\theta})^{2}+(1+2 \Gamma / A)\left[A^{2}(1+2 \Gamma / A)+2 \Omega^{2}\right] \text {. }
$$

The radiation force, Eq. (26), becomes

$$
\vec{F}=-\frac{\hbar q A \Omega^{2} \nabla \theta+\hbar(\Delta+\dot{\theta}) \nabla \Omega^{2}}{4(\Delta+\dot{\theta})^{2}+q\left(q A^{2}+2 \Omega^{2}\right)}
$$

where $q=1+2 \Gamma / A$.

In a plane running wave, $E(\vec{x}, t)=E \cos \{\vec{k} \cdot \vec{x}-\omega t-\phi(t)\}$, the radiation force reduces to

$$
F=\frac{q A \Omega^{2} \hbar \vec{k}}{4(\Delta-\vec{k} \cdot \vec{r})^{2}+q\left(q A^{2}+2 \Omega^{2}\right)} .
$$


Near resonance $\left[(\Delta-\vec{k} \cdot \dot{r})^{2}<q A^{2} / 4\right]$ this force is less than, and off resonance $\left[(\Delta-\vec{k} \cdot \dot{\vec{r}}\rangle^{2}>q A^{2} / 4\right]$ is greater than, the corresponding force without fluctuations. The running wave force can be written as $\vec{F}=(A / 2)(\bar{w}+1) \hbar \vec{k}=A P_{2} \hbar \vec{k}$, where $P_{2}=(\bar{w}+1) / 2$ is the probability that the upper atomic level is occupied. Thus, with or without fluctuations, the force is simply the rate of spontaneous emission $\mathrm{AP}_{2}$ times the momentum $h \vec{k}$ per incident photon ${ }^{5}$.

In a general standing wave, $E(\vec{x}, t)=E(\vec{x}) \cos \{\omega t+\phi(t)\}$, the steady-state ladiation force takes the form

$$
\vec{F}=-\frac{\hbar \Delta \nabla \Omega^{2}}{4 \Delta^{2}+q\left(q A^{2}+2 \Omega^{2}\right)} .
$$

Here the force is always weaker with fluctuations than without. The standing wave force is derivable from a potential

$$
\begin{aligned}
& \vec{F}=-\nabla U, \\
& U=(\hbar \Delta / 2 q) \ln \left[4 \Delta^{2}+q\left(q A^{2}+2 \Omega^{2}\right)\right] .
\end{aligned}
$$

Since the minimum scale size of inhomogeneities of the field $E(\vec{x}, t)$ is of order $\lambda$, the maximum frequency of $\dot{\theta}(t)$ or $\Omega(t)$, for an atom moving with velocity $v$, is of order $k v$, where $k=2 \pi / \lambda$. A sufficient condition for the validity of the steady-state approximation is that this frequency be small compared to the smallest relaxation rate in the Bloch equatiors, i.e. 
This condition is not necessary for a plane running wave because $\nabla \theta=-\vec{k}$ and $\Omega$ are independent of position, but in many cases of practical interest, e.g. a standing wave, condition (36) is necessary, and this places a rather severe constraint on the range of velocity that can be treated within the steady-state approximation.

\section{ATOMIC MOTION IN BROAD-}

\section{SPECTRUM RADIATION}

When the spectral width $\Gamma$ of the applied field is large ( $\Gamma \gg A)$, the transverse relaxation rate $R_{2}^{\prime}=(A+2 \Gamma) / 2$ greatly exceeds the longitudinal relaxation rate $R_{1}=A$. In this 1 imit, and when $k v \ll(A+2 \Gamma) / 2$, $\dot{\bar{u}}$ and $\dot{\bar{v}}$ may be set equal to zero in the first two Bloch equations, Eqs. (25a) and (25b), to obtain

$$
\begin{aligned}
& \bar{u}=\frac{4(\Delta+\dot{\theta}) \operatorname{si}}{4(\Delta+\dot{\theta})^{2}+(A+2 \Gamma)^{2}}, \\
& \bar{v}=\frac{2(A+2 \Gamma) \Omega \bar{w}}{4(\Delta+\dot{\theta})^{2}+(A+2 \Gamma)^{2}},
\end{aligned}
$$

and using this value for $\bar{v}$, the third Bloch equation,

$$
\dot{\bar{W}}=-\left\{A+\frac{2(A+2 \Gamma) \Omega^{2}}{4(\Delta+\dot{\theta})^{2}+(A+2 \Gamma)^{2}}\right\} \bar{W}-A \text {, }
$$

can be integrated without difficulty. Upon substituting (37) and (38) into (26), the radiation force becomes

$$
\vec{F}=\frac{\hbar(A+2 \Gamma) \Omega^{2} \nabla \theta+\hbar(\Delta+\dot{\theta}) \nabla \Omega^{2}}{4(\Delta+\dot{\theta})^{2}+(A+2 \Gamma\}^{2}} \text {. }
$$


This approach is an improvement on the steady-state approximation because the constraint $k v<<(A+2 \Gamma) / 2$ is much weaker than $k v<<A$ when $\Gamma \gg>A$.

As an illustration of the broad-spectrum theory, we shall apply Eqs. (39) and (40) to the motion of an atom in the standing wave $E(x, t)=2 E_{0} \cos (k x) \cos \{\omega t+\phi(t)\}$. It is, by now, well known that the time-averaged radiation force acting on a uniformly moving atom ( $x=v t)$ in a weak $\left(\Omega_{0}=\mu E_{0} / h<<A\right)$ coherent $(\phi(t)=0)$ standing wave is the sum of the radiation forces associated with each of the two running wave components $\left(E_{0} \cos \{k x-\omega t\}\right.$ and $\left.E_{0} \cos \{k x+\omega t\}\right)$, as if those components acted independent $1 y^{38}$,

$$
\begin{aligned}
\bar{F} & =\frac{A \Omega_{0}^{2} \hbar k}{4\left(\Delta-k !^{\prime 2}+A^{2}\right.}-\frac{A \Omega_{0}^{2} \hbar k}{4(\Delta+k v)^{2}+A^{2}} \\
& =\frac{16 A \Omega_{0}^{2} h k^{2} v \Delta}{\left[4(\Delta-k v)^{2}+A^{2}\right]\left[4(\Delta+k v)^{2}+A^{2}\right]}
\end{aligned}
$$

This force is a positive quantity times $v \Delta$, and hence, if the standing wave is tuned below resonance $(\Delta<0)$, the force opposes the velocity and the atomic motion is damped. It was first suggested by Hänsch and Schawlow ${ }^{8}$, and later by Ashkin ${ }^{19}$ and others, that such a force might be used to $\operatorname{cool}$ an atomic vapor to a very low temperature (T-1K or less) in a short period of time $\left(\Delta t \sim 10^{-5} \mathrm{~s}\right)$. Here we shall show that in a broad-spectrum standing wave a different result is obtained, and, in particular, that the radiation must be tuned above resonance $(\Delta>0)$ to obtain cooling.

For the fluctuating standing wave $E(x, t)=2 E_{0} \cos (k x) \cos \{\omega t+\phi(t)\}$, $\Omega(x)=2 \Omega_{0} \cos (k x), \theta(x)=0$, and $E q .(40)$ becomes 


$$
F=-\frac{4 \Delta \Omega_{l}^{2} \hbar k \bar{w} \sin 2 k x}{4 \Delta^{2}+(A+2 \Gamma)^{2}}
$$

With $x=v t$, the time-averaged radiation force is

$$
\bar{F}=-\Delta \Omega_{0}^{2} \hbar k\langle\bar{w}(t) \sin 2 k v t\rangle_{a v} /\left(\Delta^{2}+\Gamma^{2}\right),
$$

where wave taken the broad-spectrum limit $\Gamma \gg A$. Equation (39) becomes

$$
\dot{\bar{w}}=-\left(A+2 \alpha \cos ^{2} k v t\right) \bar{w}-A \text {, }
$$

where $\alpha=4(A+2 \Gamma) \Omega_{0}^{2} /\left[4 \Delta^{2}+(A+2 \Gamma)^{2}\right] \approx 2 \Gamma \Omega_{0}^{2} /\left(\Delta^{2}+\Gamma^{2}\right)$, and the solution is

$$
\begin{aligned}
\bar{w}(t)= & -A \int_{-\infty}^{t} \exp \left\{-(A+\alpha)\left(t-t_{0}\right)\right. \\
& \left.-(\alpha / 2 k v)\left(\sin 2 k v t-\sin 2 k v t_{0}\right)\right\} d t_{0} \\
= & -A \int_{0}^{\infty} \exp \{-(A+\alpha) s-(\alpha / k v) \text { sinkvs } \cos [k v(2 t-s)]\} d s .
\end{aligned}
$$

With the help of the identity 46

$$
e^{-z \cos \theta}=I_{0}(z)+2 \sum_{n=1}^{\infty}(-)^{n} I_{n}(z) \cos (n \theta)
$$

where $I_{n}(z)$ is the modified Bessel function of order $n$, the solution (45) may be written as a Fourier series,

$$
\bar{w}(t)=a_{0} / ?+\sum_{n=1}^{\infty}\left(a_{n} \cos 2 n k v t+b_{n} \sin 2 n k v t\right),
$$


where

$$
\begin{aligned}
& a_{n}=2(-)^{n+1} A \int_{0}^{\infty} e^{-(A+\alpha) s} I_{n}(\alpha \text { sinkvs } / k v) \text { cosnkvs ds, } \\
& b_{n}=2(-)^{n+1} A \int_{0}^{\infty} e^{-(A+\alpha) s} I_{n}(\alpha \text { sinkvs/kv) sin nkvs ds. }
\end{aligned}
$$

On multiplying (47) by sin2kvt and averaging over time, only the term with coefficient $b_{1}$ gives a nonvanishing contribution. Equation (43) becomes

$$
\bar{F}=-(1 / 2) \Delta s_{0}^{2} \hbar k b_{1} /\left(\Delta^{2}+\Gamma^{2}\right),
$$

and from (49)

$$
b_{1}=2 A \int_{0}^{\infty} e^{-(A+\alpha) s} I_{1}(\alpha \text { sinkvs } / k v) \text { sinkvs } d s .
$$

Since $I_{1}(z)$ is an odd strictly increasing function of $z$, it is easy to see that, for $s>0, I_{1}\left(\alpha\right.$ sinkvs/kv)sinkvs (and hence $b_{1}$ ) has the same sign as $v$. It follows that $\bar{F}$ amplifies the atomic velocity when the radiation is tuned below resonance $(\Delta<0)$, and damps the velocity when the radiation is tuned above resonance $(\Delta>0)$. This is the opposite of the prediction based on a weak coherent standing wave.

A conservative estimate of the stength of the damping force is obtained by replacing $I_{1}(\alpha 5 i n k v s / k v)$ in Eq. (51) by the first term in its power series expansion, namely (1/2)asinkvs/kv. Higher order terms increase the magnitude of the force but do not alter the sign. The result is 


$$
\bar{F} \simeq-\frac{\Delta A \hbar k^{2} \alpha^{3} v}{2 \Gamma[A+\alpha]\left[4(k v)^{2}+(A+\alpha)^{2}\right]}
$$

In the dissipative case $(\Delta>0), \bar{F}(v)$ is maximum at $k v=\frac{-(A+\alpha)}{2}$, is minimum at $k v=\frac{A+\alpha}{2}$, and between thrse limits is approximately linear in $v$, $\bar{F}=-\Delta A \hbar k^{2} \alpha^{2} v /\left[2 \Gamma(A+\alpha)^{3}\right]$. The solution of the equation of motion $M v=\bar{F}(v)$ is then $v(t)=v(0) \exp \left\{-t / \tau_{v}\right\}$, where $\tau_{v}=2 M \Gamma(A+\alpha)^{3} / \Delta A \hbar k^{2} \alpha^{2}$ is the velocity relaxation time. To get an order of magnitude estimate of $\tau_{v}$, we take $A=10^{8} \mathrm{~s}^{-1}, \Delta=\Gamma=\Omega_{0}=10^{9} \mathrm{~s}^{-1}, M=4 \times 10^{-23} \mathrm{~g}, \lambda=0.5 \mu \mathrm{m}$, and obtain $\tau_{v} \approx 5 \times 10^{-5} \mathrm{~s}$.

Although the damping force considered here is of comparable magnitude to that considered by Hänsch and Schawlow, it must be emphasized that the former will probably be more difficult to detect experimentally because the broad-spectrum radiation required must be the result of phase and not amplitude fluctuations.

Perhaps the most important conclusion to be drawn from this work, a conclusion that follows by inspection of Eqs. (25), is that fluctuations have a substantial effect on the radiation force only when the spectral width $\Gamma$ approaches or exceeds the spontaneous rate $A$. 
Chapter 7

QUANTUM-MECHANICAL FLUCTUATIONS OF THE RESONANCE-RADIATION FORCE 
Quantum-Mechanical Fluctuations
of the Resonance-Radiation Force

\author{
R. J. Cook \\ University of California \\ Lawrence Livermore Laboratory \\ P.0. Box 5508 \\ Livermore, Cal ifornia 94550
}

\title{
Abstract
}

The influence of quantum-mechanical fluctuations of radiation pressure on atomic motion in resonant radiation is investigated theoretically. It is shown that fluctuations of the radiation force. result from interaction of the fluctuating atomic dipole moment with the applied field (induced fluctuations) as well as from random recoils that accompany spontaneous emission (spontaneous fluctuations). Atomic motion under the influence of the fluctuating radiation force is described by a Fokker-Planck equation, and this equation is applied to problems of atomic trapping and cooling. It is shown that cooling of an atomic vapor by a standing wave tuned below resonance, known to occur in a weak field, is inhibited by fluctuations in a strong field, and that fluctuations can prevent stable trapping of atoms by the dipole radiation force. 
The importance of quantum-mechanical fluctuations of the radiation force in determiliing the motion of an atom in an electromagnetic wave tas first emphasized by Einstein in 1917.7 In this early work, Einstein showed that fluctuations due to both spontaneous and induced absorption/emission processes were necessary to account for the Maxwellian distribution of atomic velocity in t.ermal equilibrium. In recent years, a number of authors have proposed methods for trapping, ${ }^{17,19}$ deflecting, ${ }^{75,28}$ and cooling ${ }^{18,19,20}$ atoms by use of the resonant light forces in tunable laser radiation. Altiough the fluctuations due to random recoils accompanying spontaneous emission (spontaneous fluctuations) have often been considered in these proposals, the fluctuations associated with induced absorption-emission processes (induced fluctuations) have usualty been ignored.

The purpose of this letter is to point out that induced fluctuations can strongly influence atomic motion in resonant radiation and, in particular, that induced fluctuations place a lower bound on the temperature achievable by radiation cooling and lead to finite, often short, confinement times for atoms in radiation traps.

In strong coherent radiation, induced atomic processes are correctly described by interaction with a classical field. The Heisenberg equations for one-dimensional motion of an atom of mass $M$ in a classically-prescribed linearly-polarized electromagnetic wave $\vec{E}(x, t)=$ $\hat{E} E(x, t)$, in the dipcie approximation, are

$$
\dot{\hat{R}}=\hat{\mathrm{P}} / M,
$$




$$
\dot{\hat{p}}=\hat{\mu} \partial E(\hat{R}, t) / \partial x \quad \text {, }
$$

where $\hat{\mu}$ is the component of the electric dipole moment operator in direction $\hat{\varepsilon}$. If the atomic wave packet is small compared to the distance over which $\partial E(x, t) / \partial x$ changes by a significant amount, the operator $\hat{R}$ in (2) may be replaced by its expectation value $R=\langle\hat{R}\rangle$, since any matrix element involving $\partial E(\hat{R}, t) / \partial x$ is only negligibly affected by this replacement when the wave packet is small.

We consider the motion of a two-level atom with internal states |1> and $\mid 2>$ of energy $E_{1}$ and $E_{2}$ respectively in a monochromatic field $E(x, t)=E(x) \cos \{\theta(x)+$ wt $\}$ with arbitrary amplitude $E(x)$ and phase $\theta(x)$. In terms of atomic operators $\hat{S}=|1><2|$ and $\hat{S}^{+}=|2><1|$, the dipole operator takes the form $\hat{\mu}=\mu\left(\hat{S}+\hat{S}^{+}\right)$, where $\mu=\langle 1|\hat{\mu}| 2\rangle$ is the transition dipole moment, taken here to be real. In the Heisenberg picture, operators $\dot{S}, \hat{S}^{+}$are rapidly varying functions of time. It is more convenient to work with slowly varying operators $\hat{\sigma}$ and $\hat{\sigma}^{+}$defined by relations $\hat{S}=\hat{\sigma} \exp \{-i[\theta+\omega t]\}$ and $\hat{S}^{+}=\hat{\sigma}^{+} \exp \{i[\theta+\omega t]\}$ respectively. Upon substituting the above expressions for $E(x, t)$ and $\hat{\mu}$ into (2), and discarding inessential terms that oscillate at twice the optical frequen ' (rotating wave approximation), the equation for atomic moment um becomes

$$
\dot{\hat{\mathrm{p}}}=\frac{\hbar}{2}\left[\frac{\partial \Omega}{\partial x} \hat{\sigma}_{1}+\Omega \frac{\partial \theta}{\partial x} \hat{\sigma}_{2}\right] \text {, }
$$


where $\hat{\sigma}_{1}=\hat{\sigma}+\hat{\sigma}^{+}, \hat{\sigma}_{2}=i\left(\hat{\sigma}-\hat{\sigma}^{+}\right)$, and $\Omega=\mu E / \hat{h}$ is the on-resonance Rabi flopping frequency of the two-level atom.

It is well known that the expectation values of operators $\hat{\sigma}_{1}, \hat{\sigma}_{2}$, and $\hat{\sigma}_{3}=\hat{\sigma}^{+} \hat{\sigma}-\hat{\sigma}_{\hat{\sigma}}^{+}$, namely $u=\left\langle\hat{\sigma}_{1}\right\rangle, v=\left\langle\hat{\sigma}_{2}\right\rangle$ and $w=\left\langle\hat{\sigma}_{3}\right\rangle$, are the components of the Bloch vector; and if the atom experiences relaxation due to spontaneous emission, these components satisfy the optical Bloch equations 12

$$
\begin{aligned}
& \dot{u}=(\Delta+\dot{\theta}) v-\frac{1}{2} A u, \\
& \dot{v}=-(\Delta+\dot{\theta}) u+\delta w-\frac{1}{2} A v, \\
& \dot{w}=-\Omega v-A(w+1),
\end{aligned}
$$

where $\Delta=\omega-\omega_{0}$ is the detuning frequency $\left(\omega_{0}=\left(E_{2}-E_{1}\right) / \hbar\right)$, and $A$ is the Einstein spontaneous emission coefficient. The expectation value of (3) is the mean radiation force acting on the atom

$$
F=\frac{\hbar}{2}\left[\frac{\partial \Omega}{\partial x} u+\Omega \frac{\partial \theta}{\partial x} v \vec{\jmath} .\right.
$$

Equations (4) and (5) were recently derived using a slightly different approach, and were applied to a number of problems of current interest.

Equation (3) indicates that the force $\hat{F}=\hbar\left[(\partial \Omega / \partial x) \hat{\sigma}_{1}+\Omega(\partial \theta / \partial x) \hat{\sigma}_{2}\right] / 2$ should be regarded as an operator. Using the fact that operators $\hat{\sigma}_{1}$ and $\hat{\sigma}_{2}$ satisfy the anticommutation relations $\hat{\sigma}_{i} \hat{\sigma}_{j}+\hat{\sigma}_{j} \hat{\sigma}_{i}=2 \delta_{i j}$, it is readily shown that the mean square force has the form 


$$
\left.<\hat{F}^{2}\right\rangle=\frac{\hbar^{2}}{4}\left[\left(\frac{\partial \Omega}{\partial x}\right)^{2}+\Omega^{2}\left(\frac{\partial \theta}{\partial x}\right)^{2}\right]
$$

which is independent of the state of the atom. Comparing (6) with (5), and noting that $u$ and $v$ are constrained only by the relation $u^{2}+v^{2} \leq 1$, it is easy to see that the rms fluctuation of the radiation force, $\left(<\hat{F}^{2}>-F^{2}\right)^{1 / 2}$, can exceed the mean force $F$. This is a clear indication that quantum fluctuations of the radiation force will have a significant influence on atomic motion.

The motion of an atom under the influence of a fluctuating force is described by the Fokker-planck equation. If in time $\Delta t$ the force gives rise to a mean increment of momentum $\langle\Delta P\rangle$ and a mean square fluctuation of momentum about the mean increment $\left\langle[\Delta P-\langle\Delta P\rangle]^{2}\right\rangle$, if the limits

$$
\begin{aligned}
& \lim _{\Delta t \rightarrow 0} \frac{\langle\Delta P\rangle}{\Delta t}=F, \\
& \operatorname{Lim}_{\Delta t \rightarrow 0} \frac{\left.\langle\Delta P-\langle\Delta P\rangle]^{2}\right\rangle}{\Delta t}=20,
\end{aligned}
$$

exist, and if all other moments per unit time, e.g. $<[\Delta x-<\Delta x\rangle][\Delta P-$ $<\Delta P>]>/ \Delta t$, vanish in this limit, the Fokker-Planck equation takes the form $^{48}$

$$
\frac{\partial f}{\partial t}=-\frac{P}{M} \frac{\partial f}{\partial x}-\frac{\partial}{\partial P}[\mathrm{Ff}]+\frac{\partial^{2}}{\partial P^{2}}[D f],
$$

where $f(x, P)$ is the distribution function in phase space. 
The 1 imit (7) is clearly the mean radiation force of Eq. (5). To calculate $D$ we proceed as follows. With $\hat{F}$ defined as the right-hand side of (3), the increment of momentum is $\Delta \hat{P}=\int_{t_{0}}^{t} \hat{F}(s) d s$, the mean increment is $\langle\Delta \hat{\mathrm{P}}\rangle=\int_{\mathrm{t}}^{\mathrm{t}} \mathrm{F}(\mathrm{s}) \mathrm{ds}$, and

$$
\begin{aligned}
\left\langle[\Delta \hat{P}-\langle\Delta \hat{P}\rangle]^{2}\right\rangle= & \int_{t_{0}}^{t} \int_{t_{0}}^{t} d s_{1} d s_{2}\left[\left\langle\hat{F}\left(s_{1}\right) \hat{F}\left(s_{2}\right)\right\rangle\right. \\
& \left.-F\left(s_{1}\right) F\left(s_{2}\right)\right] .
\end{aligned}
$$

The correlation time of fluctuations of the force $\hat{F}$ is essentially the correlation time of fluctuations of the dipole moment $\hat{\mu}$, which is known to be on the order of the natural lifetime $\tau_{n}=i / A$. We consider the case in which the amplitude $\Omega$ and phase $\theta$ of the applied field (at the moving atom) are nearly constant over a natural lifetime. In this case, the field-dependent factors in the force (3) can be taken outside of the integrals in (10), and for $\Delta t=t-t_{0}$ somewhat larger than : correlation time, Eq. (10) yields

$$
\begin{aligned}
& 2 D_{I} \approx \frac{\leq\left[\Delta \hat{P}-\langle\Delta \hat{P}>]^{2} \geq\right.}{\Delta t} \\
& \approx \int_{-\infty}^{\infty} d s[\hat{F}(0) \hat{F}(s)>-F(0) F(s)] \\
&= \frac{\hbar^{2}}{4}\left\{\left(\frac{\partial \Omega}{\partial x}\right)^{2} \int_{-\infty}^{\infty} U_{1}(s) d s\right. \\
&+\Omega^{2}\left(\frac{\partial \theta}{\partial x}\right)^{2} \int_{-\infty}^{\infty} V_{2}(s) d s \\
&\left.+\Omega \frac{\partial \Omega}{\partial x} \frac{\partial \theta}{\partial x} \int_{-\infty}^{\infty}\left[V_{1}(s)+U_{2}(s)\right] d s\right\},
\end{aligned}
$$


where

$$
\begin{aligned}
& v_{1}(t)=\left\langle\hat{\sigma}_{1}(0) \hat{\sigma}_{1}(t)\right\rangle-u(0) u(t), \\
& v_{1}(t)=\left\langle\hat{\sigma}_{1}(0) \hat{\sigma}_{2}(t)\right\rangle-u(0) v(t), \\
& v_{2}(t)=\left\langle\hat{\sigma}_{2}(0) \hat{\sigma}_{1}(t)\right\rangle-v(0) u(t), \\
& v_{2}(t)-\left\langle\hat{\sigma}_{2}(0) \hat{\sigma}_{2}(t)\right\rangle-v(0) v(t),
\end{aligned}
$$

we have set $t_{0}=0$ for simplicity, and the subscript on $D_{I}$ indicates that this is the contribution from induced fluctuations.

The theory of dipole correlation functions, such as (12), is by now fairly standard, having been developed in connection with the problem of resonance fluorescence by Mollow 49 and others. ${ }^{50,51}$ According to this theory, if we introduce two additional correlation functions

$$
\begin{aligned}
& w_{1}(t)=\left\langle\hat{\sigma}_{1}(0) \hat{\sigma}_{3}(t)\right\rangle-u(0) w(t), \\
& w_{2}(t)=\left\langle\hat{\sigma}_{2}(0) \hat{\sigma}_{3}(t)\right\rangle-v(0) w(t),
\end{aligned}
$$

then each of the sets of correlation functions $u_{i}, v_{j}, W_{i}(i=1,2)$ satisfy homogeneous Bloch equations

$$
\begin{aligned}
& \hat{v}_{i}=(\Delta+\dot{\theta}) V_{i}-\frac{1}{2} A U_{i}, \\
& \dot{v}_{i}=-\left(\Delta+\dot{\theta} U_{i}+\Omega W_{i}-\frac{1}{2} A V_{i},\right. \\
& \dot{u}_{i}=-\Omega V_{i}-A W_{i} .
\end{aligned}
$$


105

The remainder of the calculation is given in outline. First, it is clear from (11) that only the real parts of $U_{i}, V_{i}, W_{i}$ are significant.

The real parts are even functions of $t$, so the integrals in (11) are replaced by twice the integrals from 0 to $\infty$. Next, integrating Eds. (14) from 0 to $\infty$, we obtain an algebraic set of equations for $\int_{0}^{\infty} u_{i} d s$, $\int_{0}^{\infty} v_{i} d s$, and $\int_{0}^{\infty} w_{i} d s$ involving initial conditions $u_{i}(0)$, $V_{i}(0)$, and $w_{j}(0)$. The initial conditions are obtained from (12) and (13) by using $\hat{\sigma}_{\mathbf{i}} \hat{\sigma}_{j}=\delta_{\mathbf{i j}}+\mathbf{j}_{\mathbf{i j k}} \hat{\sigma}_{k}$ and the steady-state solution of the Bloch equations (4). Finally, tie algebraic equations for $\int_{0}^{\infty} u_{i} d s$ etc. are solved, and the results are used in (11) to obtain

$$
\begin{aligned}
D_{I}= & \frac{\hbar^{2}}{2}\left\{( \frac { \partial \Omega } { \partial x } ) ^ { 2 } \left[A\left(\left[4 \Delta_{e}^{2}+A^{2}\right]^{2}-8 \Delta_{e}^{2} \Omega^{2}\right)\right.\right. \\
& +2 A^{-1} \Omega^{2}\left(3 A^{4}+6 A^{2} \Omega^{2}+4 \Omega^{4}\right) \\
& -\Omega \frac{\partial \Omega}{\partial x} \frac{\partial \theta}{\partial x}\left[16 \Delta e^{2}\left(2 A^{2}+\Omega^{2}\right)\right] \\
& +\Omega^{2}\left(\frac{\partial \theta}{\partial x}\right)^{2}\left[2 \Omega ^ { 2 } A \left(12 \Delta_{e}^{2}-A^{2}+22^{2}\right.\right. \\
& \left.\left.+A\left(4 \Delta_{e}^{2}+A^{2}\right)^{2}\right]\right\}\left[4 \Delta_{e}^{2}+A^{2}+2 \Omega^{2}\right]^{-3}
\end{aligned}
$$

where $\Delta_{e}=\Delta+\dot{\theta}$ is an effective retuning. In a weak field $(\Omega \rightarrow 0)$, (15) becomes

$$
D_{I}=\frac{\hbar^{2} A}{2\left(4 \Delta^{2}+A^{2}\right)}\left[\left(\frac{\partial \Omega}{\partial x}\right)^{2}+\Omega^{2}\left(\frac{\partial \theta}{\partial x}\right)^{2}\right],
$$


and in a strong field $(\Omega+\infty)$

$$
D_{I}=\frac{\hbar^{2}}{2 A}\left[\left(\frac{\partial \Omega}{\partial x}\right)^{2}+\frac{A^{2}}{2}\left(\frac{\partial \theta}{\partial x}\right)^{2}\right],
$$

where the second term in (17) is usually negligible when $\partial \Omega / \partial x \neq 0$.

The term containing $D$ in the Fokker-Planck equation represents diffusion of momentum. In addition to the coefficient of induced diffusion $D_{I}$, there is a well-known contribution $D_{S}$ to the total diffusion coefficient $D=D_{,}+D_{S}$, resulting from random recoils associated with spontaneous emission. If the atoms dipole moment is transverse to the $x$-direction, it is readily shown that the coefficient of spontaneous diffusion is $D_{S}=\frac{1}{5} A\left(\hbar \omega_{0} / c\right)^{2} P_{2}=\frac{1}{10} A\left(\hbar \omega_{0} / c\right)^{2}(w+1)=$ $\left.A\left(\hbar_{\omega_{0}} \Omega\right)^{2} / 5 c^{2} \tau_{4 \Delta} \Delta_{e}^{2}+A^{2}+2 \Omega^{2}\right]$, where $P_{2}=\frac{1}{2}(w+1)$ is the probability that the upper atomic level is occupied, and we have taken the steady-state value of $w$ to obtain the final form. In a strong field $D_{S}$ saturates to the value $D_{S}=\frac{1}{10} A\left(\hbar_{0} / c\right)^{2}$.

We now consider a few simple examples to illustrate the above theory. In a strong resonant traveling wave $E(x, t)=E \cos \left\{k x-\omega_{0} t\right\}$, the saturated radiation force 38 is $F=\frac{1}{2} A \hbar k\left(k=\omega_{0} / C\right)$, the coefficient of induced diffusion, Eq. (17), is $D_{I}=\frac{1}{4} A(\hbar k)^{2}$, the spontaneous coefficient is $D_{S}=\frac{1}{10} A(\hbar k)^{2}$, and the total momentum diffusion coefficient $D=7 \mathrm{~A}\left(\hbar_{\mathrm{K}}\right)^{2} / 20$ is 3.5 times that which might have been expected on the basis of spontaneous recoils alone. 
In a strong standing wave $E(x, t)=2 E_{0} \cos k x \cos \omega t$ $\left(\Omega=2 \Omega_{0} \cos \mathrm{kx}, \Omega_{0}=\mu E_{0} / \hbar, \theta=0\right)$, the dipole force acting on a slowly moving atom is $F=-\hbar \Delta \partial \Omega^{2} / \partial x /\left[4 \Delta^{2}+A^{2}+2 \Omega^{2}\right]$, the induced diffusion coefficient, Eq. (17), is $D_{I}=\frac{1}{2} \hbar^{2}(\partial \Omega / \partial x)^{2} / A$ $\sim \Omega_{0}^{2}(\hbar k)^{2} / A$, and $D_{S}$ is negligible compared to $D_{I}$. On resonance $(\Delta=0)$ the dipole force vanishes and atomic motion is dominated by induced momentum diffusion. In a standing wave, induced diffusion results from a splitting of the atomic trajectory after each spontaneous transition to the ground state, and is closely related to the optical Stern-Gerlach effect. 24,52

The cooling or heating of an atomic vapor by resonant radiation is calculated as follows. Generally the radiation force consists of a part $F^{\prime}=-\partial V(x) / \partial x$ derivable from a potential and a part $F$ not derivable from a potential. Multiplying the Fokker-Planck equation, (9), by $P^{2} / 2 M+V(x)$, integrating over phase space, and performing some integrations by parts (assuming $f=0$ at $|x|=\infty$ and $|P|=\infty$ ), we obtain the relation

$$
E=M^{-1} \iint\left[D+P F{ }^{--}\right] f d x d P
$$

for the rate of change of the mean translational energy $E=\iint\left[P^{2} / M+V\right]_{f d x d p}$ of the atom. For example, in a weak $(\Omega \ll A)$ standing wave, tuned below resonance by the amount $\Delta=-\frac{1}{2} A$, the total diffusion coefficient is $D \approx 7 \Omega_{0}^{2}(\hbar k)^{2} / 10 A$, the radiation damping force, for small atomic velocity, is $F^{38}=-\left(2 \Omega_{0}^{2} \hbar k^{2} / A^{2}\right) V$, and (18) 
states that the energy $E$ dissipates until $\overline{D^{+P F}=-}=0$ or $\frac{1}{2} \overline{M_{v}^{2}} \approx 7 \hbar A / 40$. For $A=10^{8} s^{-1}$ this corresponds to a temperature $T$ \& $10^{-4} K$. However, as the intensity increases, $D$ increases without bound, because of induced diffusion, while the strength of the dissipative force $F^{\cdots}$ is certainly bounded by $\frac{1}{2}$ Afik. Therefore induced fluctuations inhibit cooling in a strong standing wave.

Finally, consider radiation trapping of atoms by the dipole force in a strong field $\left(\Omega \gg\right.$ A) of amplitude $\Omega(x)=\Omega_{0} \exp \left\{-x^{2} / w_{0}\right\}$, e.g. transverse trapping in a Gaussian laser beam. The potential energy of the dipole forcc, ${ }^{38} v=\frac{1}{2} \hbar \Delta \operatorname{Ln}\left(1+\Omega^{2} / 2 \Delta^{2}\right)$, assumes its minimum value $v_{\min }=-0.28 \hbar \Omega_{0}$ for $\Delta=-0.35 \Omega_{0}$. Since no dissipative force acts on the atom, (18) states that $\dot{E}=\bar{D} / M$, and (17) yields the estimate $D=D_{I}+D_{S}$ it $\frac{1}{10} \hbar^{2} \Omega_{0}^{2} / A w_{0}^{2}+\frac{1}{10} A(\hbar k)^{2}$ for the average $D$ in the we11. A trapped atom gains energy from fluctuations and escapes from the well in a time of order $\Delta t=-V_{\min } / \dot{E}=2.8 \hbar \Omega_{0} M /\left[A \hbar^{2} k^{2}+\hbar^{2} \Omega_{0}^{2} / A w_{0}^{2}\right]$. For a sodium atom in a Gaussian beam of radius $w_{0}=10 \mu \mathrm{m}$ and power $50 \mathrm{~mW}$, tuned to the $3^{2} \mathrm{~S}_{1 / 2}+3^{2} \mathrm{P}_{3 / 2}$ transition, the confinement time is $\Delta t \approx 10^{-4} \mathrm{~s}$. This is about two orders of magnitude less than the value obtained if only spontaneous fluctuations are considered.

The above examples indicate that quantum-mechanical fluctuations of the resonance-radiation force will be an important consideration in the design of experiments to trap or cool atoms and molecules. 
Chapter 8

OVERV IEW OF EXPERIMENTS ON ATOMIC MOTION IN RESONANT RADIATION 
Deflection of an atomic beam by momentum transfer from resonant traveling radiation was first observed by $R$. $\mathrm{Frish}^{1}$ in 1933 . Because of the low spectral purity of radiation sources available at the time, the deflections achieved in this experiment were very small, and of little practical interest. An improved experiment to measure the deflection of an atomic beam by the spontaneous radiation force, but still using classical sources of radiation (discharge lamps), was made by J. L. Picque and J. L. Vialle $e^{2}$ in 1972. In this experiment atomic beams of sodium and cesium were deflected by transverse illumination, and the beam profile, with and without illumination, was measured with a hot-wire probe. Bean deflection and beam spreading were found to be in reasonable agreement with theory. The magnitude of deflections $\left(\theta \sim 10^{-5} \mathrm{rad}\right)$ was again quite small.

With the invention of the Laser in the early 1960's and, in particular, with the development of high power tunable lasers in the early 1970's the experimental situation changed dramatically. Numerous proposals were made involving applications of the radiation force to problems of isotope separation, atomic trapping and cooling, neutral-atom acceleration, and atomic-beam-deflection spectroscopy; and several experiments were carried out using laser radiation. Some of these experiments will now be listed.

R. Schieder, H. Walther, and L. Woste ${ }^{3}$ demonstrated deflection of a sodium atomic beam by the radiation pressure of a tunable $\mathrm{cw}$ dye laser, the observed deflections being about 60 times larger than those achieved in the experiments of Frish and of Picque and Vialle. J. E. Bjorkholm, 
A. Ashkin, and D. B. Pearson ${ }^{53}$ showed that the resonance-radiation force of light propagating axially down a tube filled with sodium vapor causes a macroscopic pressure increase along the length of the tube. Specifical1y, they achieved a pressure difference of $50 \%$ in a tube of $20 \mathrm{~cm}$ length and they suggested that an isotopically selective application of this effect might be used for isotope enrichment. In 1973 P. Jacquint, 5. Liberman, J. L. Picque, and J. Pinard ${ }^{54}$ demonstrated that spectroscopy can be carried out by observing bean deflection as the frequency of the deflecting radiation is varied. The beam is deflected anly when the tunable radiation comes into resonance with an atomic transition. The method is capable of high resolution as demonstrated by the well resolved hyperfine structure of sodium in this experiment. In 1974 isotope separation by selective deflection of one isotopic component of an atomic beam was first achieved at Lawrence Livermore Laboratory by A. F. Bernhardt, D. E. Duerre, J, R. Simpson, and L. L. Wood ${ }^{10}$ for isotopes of barium using a tunable dye laser.

Al1 of the above experiments involve applications of the spontaneous radiation force. The first observation of the dipole radiation force was made in a recent experiment at Bell Labs by 3. E. Bjorkho $3 m$, R. R. Freeman, A. Ashkin, and D. B. Pearson ${ }^{37}$. In this experiment focusing and defocusing of a sodium atomic beam by the transverse dipole force in a copropagating Gaussian laser beam was observed when the laser radiation was tuned, respectively, below and above the Bohr transition frequency of the atoms. The strength and qualitative behavior of the dipole force were found to be in satisfactory agreenent with a theory of 
the dipole force equivalent to that developed in Chapter 5. This experiment represents an important step toward the development of radiation traps for atoms and molecules.

A first attempt to measure the induced-rate momentum transfer in a strong resonant standing wave was made very recently in the experiment of E. Arimondo, H. Lew, and Takeshi $\mathrm{Oka}^{55}$ at the Herzberg Institute of Astrophysics. A CW laser beam was reflected back on itself by a mirror to form a standing wave, a well colimated atomic beam of sodium was propagated across the standing wave, and the atomic-beam profile was measured on the downstream side of the interaction region using a hot-wire detector. By blocking the mirror the standing wave was replaced by a running wave, and in this way a comparison of beam deflection and spreading in standing and running waves of comparable intensity was made without altering the alignment of the atomic beam and detector. At the maximum laser power level used in this experiment $(100 \mathrm{~mW})$ the induced rate $\Omega$ was calculated to be about 40 times the spontaneous rate $A$, and therefore it was expected that deflection processes due to momentum transfer at these two different rates would be clearly distinguishable. With the mirror blocked only a rather small deflection was measured, while with the mirror unblocked, to form a standing wave, much larger deflections were obtained. This appears to be a c?ear indication that momentum transfer at the induced rate was observed in this experiment. However, quantitative agreement between theory and experiment has not yet been achieved. The beam spreading measured in the experiment is smaller by a factor of at least four than the theoretical predictions of Chapters 2 
and 4 , and the discrepancy may, in fact, be much larger due to uncertainties, in the experimental conditions. Several factors have been identified that might explain the discrepancy. First off, the theoretical treatments of atomic motion in a resonant standing wave presented in Chapters 2 and 4 neglect st ntaneous emission, while the interaction time in the experiment was definately longer than the natural lifetime of the suisum $\mathrm{D}_{2}$ transition. This, however, cannot completely rejoive the discrepancy because, according to theory, the beam spreading that should have occured in a single natural lifetime, during which the theory is expected to be valid, is already several times that observed in the experiment. The most probable explanation of the discrepancy is that the alignment of the atomic and optical beams was not sufficiently precise to observe the full deflection predicted by theory. According to Chapter 4, a standing wave can deflect an atom from an initial direction $(\theta=0)$ that is parallel to the planes of maximum and minimum intensity of the standing wave to a final direction that makes an angle $\theta_{\max }=$ $2(M / / \Omega)^{3 / 2} / P_{Z}$ with respect to these $p l a n e s$. If the atom enters the standing wave at an angle greater than $\theta_{\max }$ little deflection is expected. In the experiment the angle $\theta_{\max }$ is approxiniately 0.6 degrees, and hence the directions of propagation of the atomic and optical beams should have been orthogonal to or: another to an accuracy better than $0.6^{\circ}$ in order to observe the beam spreading predicted by theory. In a private communication Dr. Oka states that no attempt was made to make the atomic and laser beams accurately perpendicular and the misalignreiit could have been more than $1.5^{\circ}$. In view of this uncertainty 
of alignment, results of the present experiment must be regarded as inconclusive. Or. Oka states that he will try to repeat the experiment, with improved alignment, as soon as possible. 
115

Chapter 9

CONCLUSIONS AND RECOMMENDATIONS FOR FUTURE WORK 
The work reported here began with an attempt to understand the mechanics of atomic motion in a resonant standing light wave as a possible method of laser isutope separation. However, it soon became evident that existing theories of atomic motion in resonant radiation were not sufficiently well developed to answer a number of questions raised by this problem. The emphasis of the research therefore naturally switched from the problem of isotope separation by photodeflection to the development of more basic aspects of the theory of atomic motion in electromagnetic radiation. The author believes that the contributions to this theory contained in Chapters 3 through 7 now provide a fairly complete and quantitatively correct description of the influence of an electromagnetic wave on the translational motion of an atom, and that answers to a wide class of problems involving the resonance-radiation force can now be obtained by straight forward application of the results of these chapters. In particular, it is now possible to answer, by computer calculations, many of the questions encountered in connection with the problem of isotope separation by photodeflection in a resonant standing wave. This work is presently being pursued by the author in collaboration with Dr. A. Bernhardt and Dr. B. Shore at the Lawrence Livermore Laboratory.

It might be possible to develop a more general theory of atomic motion in electromagnetic radiation than that considered here, based on the reduced density matrix for the internal and translational motions of an atom interacting with the quantized electromagnetic field. Such a theory would address, in addition to problems of atomic motion in coherent applied fields, problems of nonequilibrium quantum statistical 
mechanics such as (1) atomic motion in partially coherent light and (2) relaxation of the atomic velocity distribution to the Maxwell distribution due to interaction with black body radiation. This is a problem for the future. 
178

ACKNOWLEDGMENTS

The author wishes to express his sincere appreciation to Professor Edward Teller for the guidance he provided, for his continued interest in this work, and for many fruitful discussions. It is a pleasure to thank Dr. A. F. Bernhardt for posing the problem that eventually lead to this research, and thanks are due also to Professor J. H. Eberly, Dr. B. W. Shore, and Dr. P. Milonni for helpful and stimulating conversations during the course of this work. 


\section{REFERENCES}

1. R. Frish, Z. Physik 86, 42 (1933).

2. J. L. Picque and J. L. Vialle, Opt. Commun. 5, 402 (1972).

3. R. Schieder, H. Walther, and L. Wöste, Opt. Commun. 5, 337 (1972).

4. A. F. Bernhardt, App 1. Phys. 34, 19 (1976).

5. A. Ashkin, Phys. Rev. Letters 25, 1321 (1970).

6. P. L. Kelley, N. M. Kroll, and C. K. Khodes, Upt. Commun. 16, 172 (1976).

7. M. H. Mittleman, K. Rubin, R. H. Callender, and J. I. Gersten, Phys. Rev. 16, 583 (1977).

8. I. Nebenzahl and A. Szöe, App1. Phys. Letters 25, 636 (1974).

9. A. P. Kazalissev, Sov. Phys. - JETP 39, 784 (1974), 40, 825 (1975).

10. A. F. Bernhardt, D. E. Duerre, J. R. Simpson, and L. L. Wood, Appl. Phys. Letters 25, 617 (1974).

11. A. F. Bernhardt, D. E. Duerre, J. R. Simpson, and L. L. Wood, Opt. Commun. 16, 166 (1976).

12. L. Allen and J. H. Eberly, Optical Resonance and Two-Level Atoms, (John Wiley and Sons, New York, 1975).

13. E. Hansen, Am. Math. Monthly 73, 143 (1966).

14. G. N. Watson, A Treatise on the Theory of Besse1 Functions, (Cambridge University Press, 1966), p. 37.

15. J. W. Goodman, Introduction to Fourier Optics, (McGraw-Hill, New York, 1968), p.69.

16. G. A. Delone, V. A. Grinchuk, A. P. Kazantsev, and G. I. Surdutovich, Opt. Commun. 25. 399 (1978). 
17. A. Ashkin, Phys. Rev. Letters 24, 156 (1970).

18. T. W. Hansch and A. L. Schawlow, Opt. Commun. 13, 68 (1975).

19. A. Ashkin, Phys. Rev. Letters $\underline{40}, 729$ (1978).

20. Y. S. Letokhov, V. G. Minogin, and B. D. Pavlik, Opt. Commun. 19,72 (1976).

21. A. P. Kazantsev, Sov. Phys. JETP 36, 861 (1973).

22. A. Yu. Pusep, Sov. Phys. JETP 43, 441 (1976).

23. J. E. Bjorkholm, R. R. Freeman, A. Ashkin, and D. B. Pearson (Post deadline paper, Tenth International Quantum Electronics Conference, At lanta, 1978).

24. R. J. Cook, Phys. Rev. Letters 41, 1788 (1978).

25. A. P. Kazantsev, Usp. Fiz. Nauk 124, 113 (1978) [ Sov. Phys. Usp. 21, $58(1978)]$.

26. R. M. Hill and T. F. Gallagher, Phys. Rev. 12, 451 (1975).

27. M. Bloom, E. Enga, and H. Lew, Can. J. Phys. 45, 148 (1967).

28. R. J. Cook and A. F. Bernhardt, Phys. Rev. A 18, 2533 (1978).

29. L. I. Schiff, Quantum Mechanics, (McGraw-Hi11, New York, 1968), p.28.

30. Z. Białynicka-Birula, I. Biakynicki-Birlula, J. H. Eberly, and B. W. Shore, Phys. Rev. A 16, 2038 (1977).

31. G. BTanch, in Handbook of Mathematical Functions, edited by M. Abramowitz and I. A. Stegun (U.S. GPO, Washington, D.C., 1966), Chap. 20.

32. M. Y. Berry, The Diffraction of Light by Ultrasound. (Academic Press, New York, 1966) and references contained therein.

33. G. A. Askar'yan, Sov. Phys. JETP $\underline{15}, 1088$ (1962). 
34. S. Stenholm, Appl. Phys. 15, 287 (1978).

35. 5. Stenholm and J. Javanaien, App1. Phys. 16, 159 (1978).

36. W. H. Louisel1, Quantum Statistical Properties of Radiation, John Wiley and Sons, New York, 1973), p. 347.

37. J. E. Bjorkholm, R. R. Freeman, A. Ashkin, and D. B. Pearson, Phys. Rev. Letters 41, 1361 (1978).

38. R. J. Cook, Phys. Rev. A 20, 224 (1979).

39. H. Haken, in Quantum Optics, edited by S. M. Kay and A. Maitland (New York Academic Press, 1970), pp. 201-321.

40. M. Sargent, M. O. Scully, and W. E. Lamb, Laser Physics (Reading, Addison Wesley, 1974).

41. W. Feller, An Introduction to Probability Theory and Its Applications (New York, John Wiley and Sons, Inc. 1957), p. 229.

42. P. Avan and C. Cohen-Tannoudji, J. Phys. B 10, 155 (1977).

43. K. Wódkiewicz, Phys. Rev., in press.

44. R. F. Fox, J. Math. Phys. 13, 1196 (1972).

45. K. Wódkiewicz, J. Math. Phys. 20, 45 (1979).

46. F. W. J. 01 ver, in Handbook of Mathematical Functions, edited by M. Abramowitz and I. A. Stegun (U. S. GPO, Washington, D. C., 1970), Chap. 9.

47. A. Einstein, Phys. Zeits, 18, 121 (1917).

48. S. Chandrasekhar, Rev. Mod. Phys. 15, 1 (1943).

49. R. B. Mollow, Phys. Rev. 188, 1969 (1969).

50. J. R. Ackerhald and J. H. Eberly, Phys. Rev. D, 10, 3350 (1974).

51. H. J. Kimble and L. Mandel, Phys. Rev. A, 13, 2123 (1976).

52. P. Knight, Nature, 278, 14 (1979). 
53. J. E. Bjorkho 7m, A. Ashkin, and D. B. Pearson, Appl. Phys. Letters 27, 534 (1975).

54. P. Jacquint, S. Liberman, J. L. Picque, and J. Pinard, Opt. Commun. $\underline{8}$, 163 (1973).

55. E. Arimondo, H. Lew, and Takeshi Oka, private communication. 


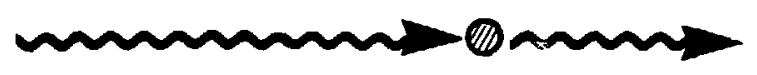

$$
\begin{array}{r}
\Delta P=0 \\
\Delta P=0 \\
\Delta P=2 \hbar K
\end{array}
$$

mommormon



$$
\triangle P=-2 \hbar K
$$

- Momentum transfer in a standing wave proceeds at the stimulated rate $\Omega$

- In a strong field $\Omega \gg A$

Figure 1. 


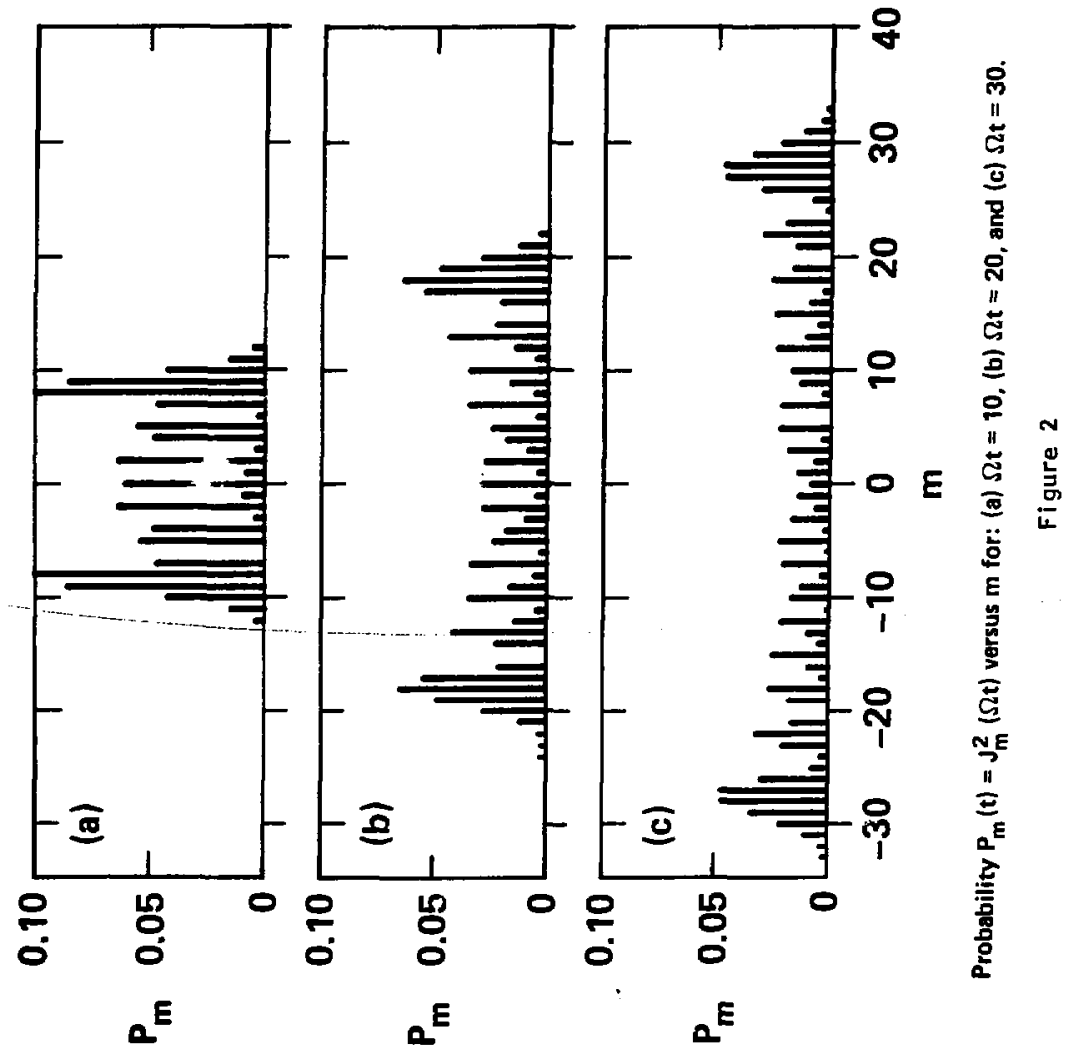




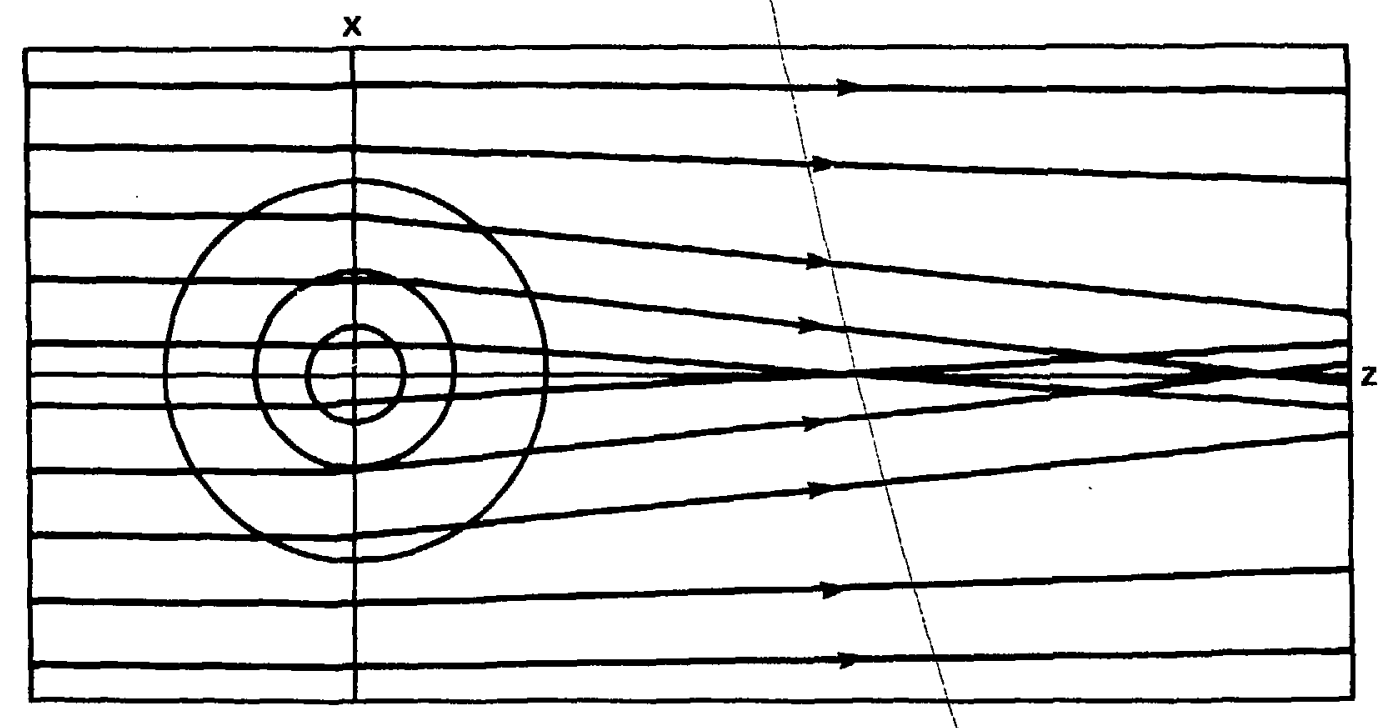

Figure 3. Focussing of atomic trajectories associated with wave function $U_{-}$. 


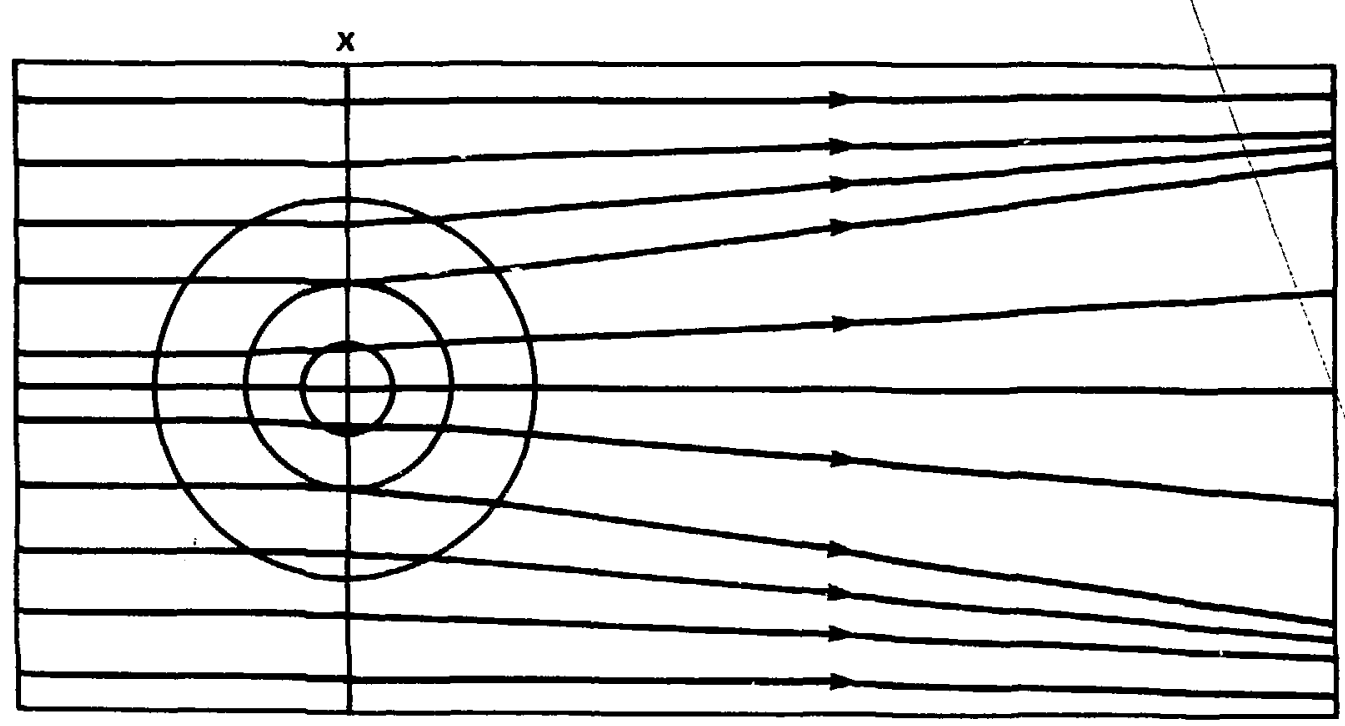

Figure 4. Defocussing of atomic trajectories associated with wave function $U_{+}$. 


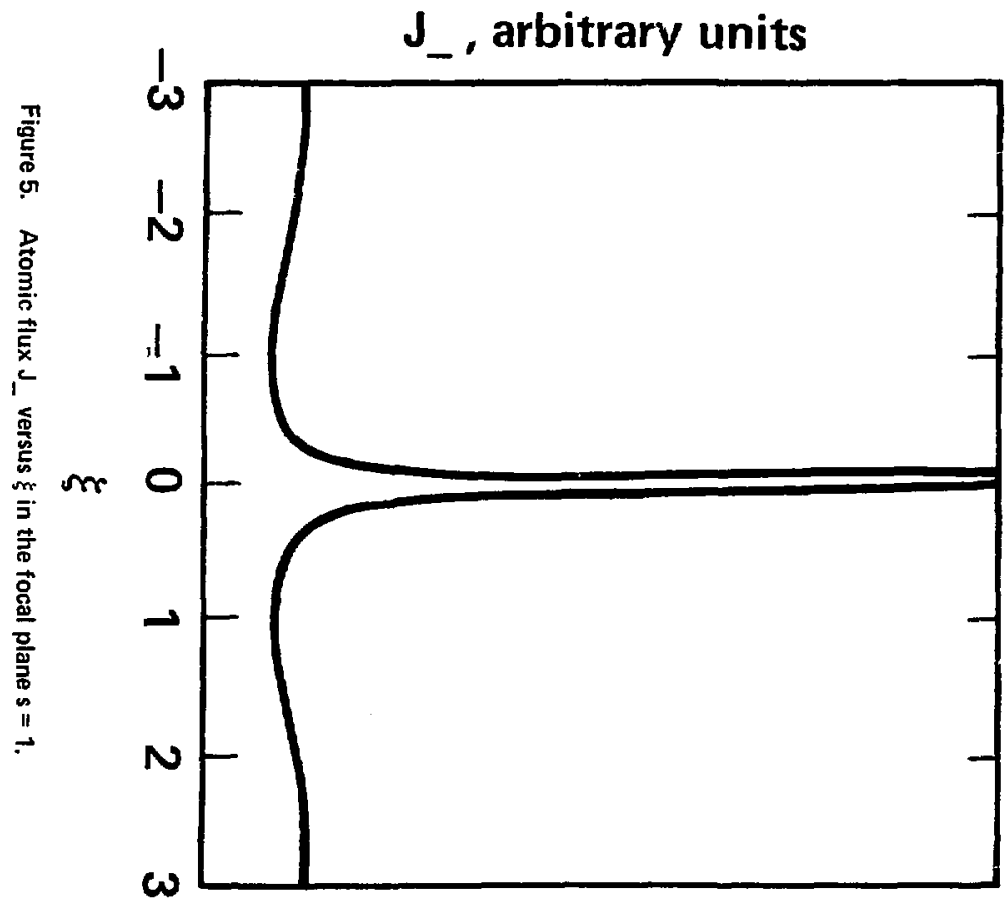




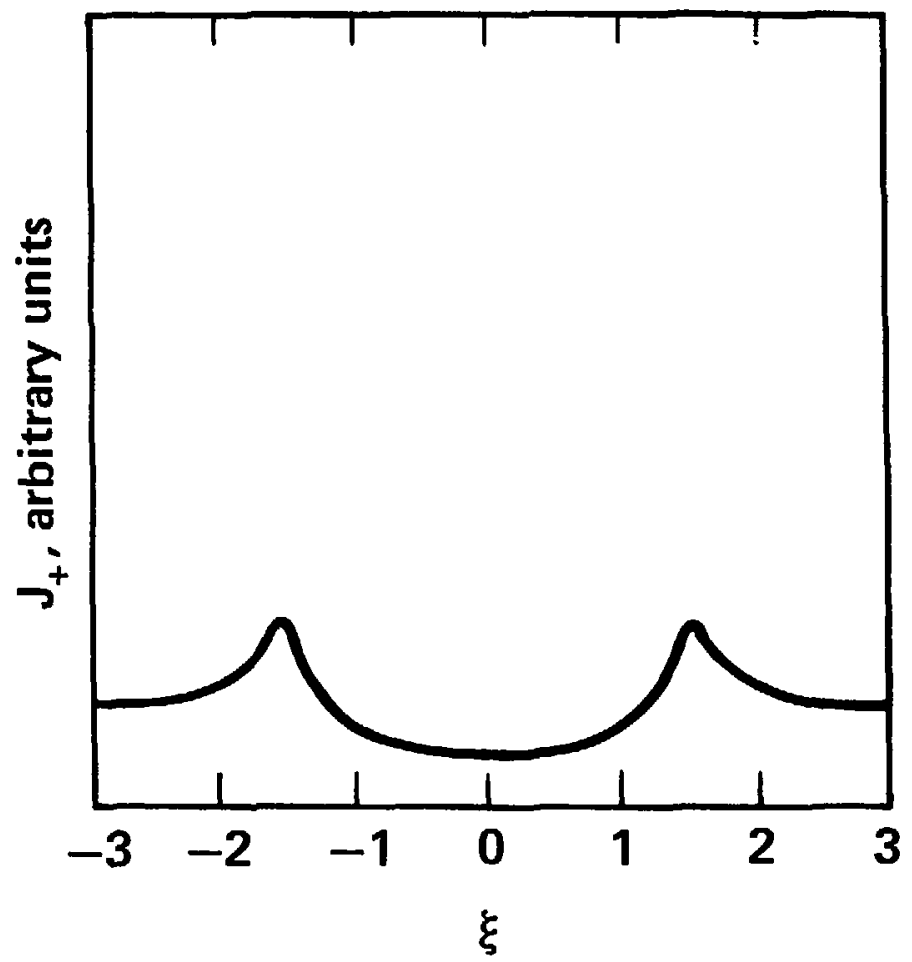

Figure 6. Atomic flux $J_{+}$versus $\xi$ in the focal plane $s=1$. 


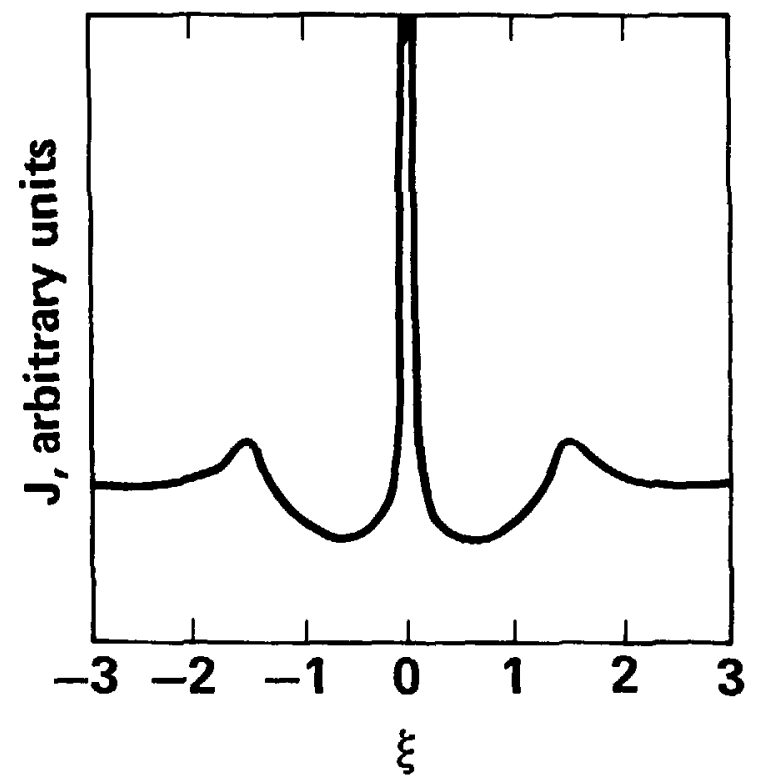

Figure 7. Total atomic flux $\mathrm{J}=\mathrm{J}_{+}+\mathrm{J}_{-}$versus $\xi$ in the focal plane $s=1$. 


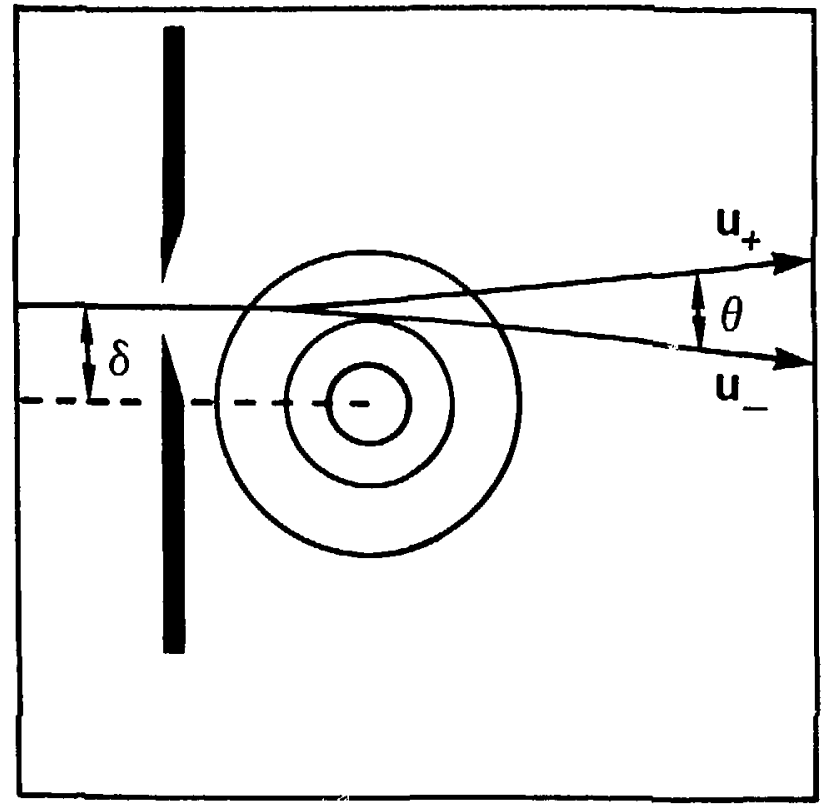

Figure 8. Splitting of an atomic beam by the amplitude gradient of the resonant field. 